\title{
Torção de Reidemeister das formas espaciais
}

esféricas 
Data de Depósito:

Assinatura:

\title{
Torção de Reidemeister das formas espaciais esféricas
}

\author{
Thiago de Melo
}

Orientador: Prof. Dr. Mauro Flavio Spreafico

Tese apresentada ao Instituto de Ciências Matemáticas e de Computação - ICMC-USP, como parte dos requisitos para obtenção do título de Doutor em Ciências - Matemática.

USP - São Carlos

Janeiro de 2009 
Não tenho um caminho novo. O que eu tenho é um jeito novo de caminhar.

(Thiago de Mello) 

À querida Alini,

com amor, carinho e gratidão.

Sem você minha jornada teria sido muito menos feliz. 

Muitas pessoas fizeram parte da minha vida durante o período dos meus estudos. A maioria apenas passou, poucas foram as que contribuíram de algum modo e recebem aqui meus agradecimentos. Desde já peço desculpas aos não citados, mas que também merecem as palavras de gratidão. Confesso que me esforcei para lembrar de todos.

Agradeço a Deus por estar presente em todos os momentos da minha vida, por ter me dado condições para que eu desempenhasse minhas obrigações da melhor forma, enfim, sempre me fazendo buscar o melhor.

Agradeço à minha família, por tudo que fizeram por mim, pela educação que deles recebi, pelo apoio nos momentos de preocupação, pela oportunidade que me deram para levar adiante meus estudos, por permitirem que eu traçasse meus próprios caminhos.

Agradeço ao meu orientador Mauro Spreafico, que desde o mestrado tem dado apoio para minha formação como pesquisador, pela grande ajuda no desenvolvimento do projeto. Também agradeço a CAPES pelo apoio financeiro, indispensável para minha permanência em São Carlos.

Agradeço aos meus professores do colégio, mesmo sabendo que a chance de se depararem com este texto é quase nula, sinto-me na vontade de dirigir-lhes meus sentimentos de gratidão. Agradeço aos professores do Departamento de Matemática da Unesp de Rio Claro, pelos conhecimentos e ensinamentos, pelas conversas e conselhos, enfim, pela minha formação (ou pelo menos, pelo início dela) como matemático. Agradeço à Suzi, por ter me encorajado a trocar a licenciatura pelo bacharelado, e à Alice pela orientação na Iniciação Científica, pelos conselhos tão valiosos nos meus momentos de dúvida, por ter sido e ainda ser uma grande amiga. Aos professores do ICMC, Valdir, Ires e Sandra, pela supervisão no PAE, importante programa para a formação de um docente. Em especial, ao professor Dide, pela valiosa contribuição neste trabalho, pelas conversas "filosóficas" nos momentos de ócio e por sempre estar disposto a ajudar.

Na vida, os colegas e amigos sempre estão presentes. Alguns, pouco, outros, mais. Mas sempre estão lá. A lista seria grande se eu fosse citar os colegas, os conhecidos e companheiros de estudo, mas caberia aqui, talvez em duas ou três linhas, a lista de amigos. Para facilitar a leitura (na verdade, para não me comprometer) agradeço, de uma só vez, aos amigos da graduação em Rio Claro, da pós-graduação em São Carlos e aos que não se enquadram nesses dois grupos. 
Encerro meus agradecimentos lembrando de uma pessoa muito especial para mim e escrevo estas palavras exclusivamente para você. Durante os quase quatro anos de doutorado, você sempre esteve comigo, às vezes no meu pensamento, por outras, ao meu lado. Não foi fácil (viu) ficar longe, passar a semana esperando a volta para casa, mas passar o fim de semana com você fez valer a pena. Você sempre me deu atenção, me ouviu quando eu precisei conversar, me deu apoio para superar as dificuldades fora dos estudos e me ensinou que "a vida não é só matemática". Poderia escrever muito, muito mais. Mas não é necessário. O que você representa para mim, você sabe muito bem. Obrigado, querida Alini.

Aos citados acima, Muito Obrigado! Thizgs de Mebo 
ABSTRACT

In this work, we study the action of the generalized quaternionic groups $\mathcal{Q}_{4 t}$ on the spheres to compute the Reidemeister torsion of the quotient spaces, which are called Quaternionic Spherical Space Forms.

Using the base of the homology defined by Ray and Singer in [27] we compute also the Ray-Singer torsion of the spheres, lens spaces and the cone over the spheres. This last one provides the disc as a particular case. For the closed manifolds we obtain the analytic torsion using the Cheeger-Müller Theorem [7, 22] and for the disc using a formula proved by Brüning and Ma in [5]. 



\section{RESUMO}

Neste trabalho, estudamos a ação dos grupos dos quatérnios generalizados $\mathcal{Q}_{4 t}$, nas esferas, com o objetivo de calcularmos a torção de Reidemeister dos espaços quocientes, chamados de Formas Espaciais Esféricas Quaterniônicas.

Calculamos a torção de Ray-Singer das esferas, dos espaços lenticulares e do cone sobre as esferas, este último fornecendo o caso particular do disco, usando a base para a homologia definida em [27]. Para as variedades fechadas, obtivemos a torção analítica por meio do Teorema de Cheeger-Müller [7, 22], e para o disco, por meio de uma fórmula provada por Brüning e Ma em [5]. 

INTRODUÇÃO

Uma forma espacial esférica é uma variedade Riemanniana conexa completa de curvatura constante positiva, ou seja, é exatamente o quociente $S^{n} / \Gamma$ da esfera pela ação de um subgrupo $\Gamma \subset O(n+1, \mathbb{R})$ de isometrias livres de ponto fixo. Desde que $S^{n} / \Gamma$ é conexa, $H_{0}\left(S^{n} / \Gamma ; \mathbb{Z}\right) \simeq \mathbb{Z}$. Como $S^{n}$ é compacta e $p: S^{n} \rightarrow S^{n} / \Gamma$ é uma cobertura regular, então $S^{n} / \Gamma$ é compacta e $H_{n}\left(S^{n} / \Gamma ; \mathbb{Z}\right)$ é isomorfo a $\mathbb{Z}$ ou $\mathbb{Z}_{2}$, dependendo se é orientável ou não orientável, respectivamente. Se $n$ é par, então as únicas formas espaciais esféricas (a menos de isometria) são a esfera e o espaço projetivo (Proposição 2.6), obtidas com $\Gamma=\{1\}$ ou $\Gamma=\{ \pm 1\}$. Se $n$ é ímpar, então $S^{n} / \Gamma$ é orientável pois, como veremos no Capítulo 2, as representações do grupo $\Gamma$ em $O(n+1, \mathbb{R})$ originam-se de representações complexas e portanto se reduzem a $S O(n+1, \mathbb{R})$. Também, o grupo das transformações cobertura (ou transformações 'deck') é $\Gamma$. Por meio da seqüência exata de homotopia de $p$ segue que $\pi_{1}\left(S^{n} / \Gamma\right) \simeq \Gamma$ e $\pi_{k}\left(S^{n} / \Gamma\right) \simeq \pi_{k}\left(S^{n}\right)$, para $k>1$.

Uma classificação completa das formas espaciais esféricas foi feita por J. Wolf em [31]. Em resumo, dados um grupo finito $G$ e uma representação ortogonal irredutível livre de ponto fixo $\sigma: G \rightarrow O(n+1, \mathbb{R})$, temos uma variedade Riemanniana conexa completa $S^{n} / \sigma(G)$, de dimensão $n$ e curvatura constante positiva. Por [31, Teorema 5.1.2], toda variedade Riemanniana conexa completa de curvatura constante positiva é isométrica a uma variedade obtida deste modo e duas representações de $G$ dão origem a variedades isométricas se, e somente se, são representações equivalentes. Portanto, o problema de classificação das formas espaciais esféricas se divide em duas partes: (1) descrever os possíveis grupos que podem ocorrer e (2) como tais grupos podem ocorrer. Ambas as partes dependem essencialmente da teoria de representação de grupos finitos. A divisão em duas partes foi observada por Vincent [29], porém a maior parte do trabalho na busca de tais grupos foi feita por Zassenhaus [32] em um outro contexto. Nas Seções 2.2 e 2.3 apresentamos um breve resumo, com alguma ênfase para o grupo dos quatérnios generalizado (também chamado de grupo quaterniônico).

A torção de Whitehead (W torção) é introduzida na teoria de homotopia simples a fim de se definir um invariante mais "sensível" do que os grupos de homotopia de um espaço. Este invariante nos permite encontrar exemplos de espaços com o mesmo tipo de homotopia mas não homeomorfos, ou seja, distingue espaços que a homotopia considera iguais. No entanto, a torção de Whitehead é 
geralmente definida para complexos acíclicos, isto é, complexos com homologia trivial. Portanto, ou iniciamos com um espaço acíclico ou então devemos encontrar uma representação do grupo fundamental que define um complexo (com coeficientes locais) que seja acíclico. Trabalharemos com espaços não acíclicos cujos complexos acíclicos (como acima) não existem. Assim, após alguns ajustes, apresentamos a definição adequada da torção nestes casos.

A torção de Reidemeister ( $\mathrm{R}$ torção) é um importante invariante topológico introduzido originalmente por Reidemeister [28], Franz [15] e de Rham [13] para classificar os espaços lenticulares (Apêndice A). Para espaços não acíclicos, a $\mathrm{R}$ torção depende da homologia. No entanto, para variedades Riemannianas, Ray e Singer [27] introduziram um invariante geométrico (RS torção). Eles usaram a estrutura Riemanniana para lidar com a dependência da homologia na $\mathrm{R}$ torção. No mesmo trabalho, procurando uma descrição analítica para a RS torção, também introduziram a torção analítica, que posteriormente tornou-se um importante invariante geométrico e tem sido estudada profundamente por vários autores, por exemplo, [3] e as referências lá citadas. A equivalência entre a RS torção e a torção analítica, conjecturada por Ray e Singer (em [26] foi calculada explicitamente a torção analítica dos espaços lenticulares, coincidindo com a $\mathrm{R}$ torção e sugerindo assim uma possível equivalência, em geral), foi posteriormente provada por Cheeger [7] e Müller [22], para variedades fechadas. Tal resultado será citado aqui por Teorema de Cheeger-Müller. Cheeger também estudou o caso de variedades com bordo, mostrando que neste caso um termo extra poderia aparecer. Posteriormente, tal termo foi explicitado por Lott e Rothenberg [18] e Lück [19], para o caso de variedades com uma estrutura de métrica produto próximo ao bordo. Recentemente, Dai e Fang [10] deram uma fórmula para a diferença da RS torção e da torção analítica em uma variedade com bordo, sem qualquer hipótese para a métrica próximo ao bordo. Nesta fórmula, alguns novos termos aparecem. No entanto, em um trabalho recente de Brüning e Ma [5] sobre métricas Ray-Singer em variedades com bordo, uma outra fórmula é dada com outra contribuição do termo do bordo. Os resultados dados no Teorema 4.13 são obtidos usando a fórmula de Brüning e Ma.

Apesar do extenso estudo e da grande literatura disponível, poucos resultados comparativos existem no estudo quantitativo, isto é, cálculos explícitos da torção analítica, como por exemplo $[12,26,30]$. Continuando nesta linha, estudamos o caso mais simples de uma variedade com bordo, ou seja, um disco. Seja $(W, g)$ uma variedade Riemanniana compacta e conexa, com bordo $\partial W$ e métrica $g$, e seja $\rho: \pi_{1}(W) \rightarrow O(k, \mathbb{R})$ uma representação ortogonal do grupo fundamental de $W$. Denotamos por $\tau_{\mathrm{RS}}((W, g) ; \rho)$ a RS torção de $(W, g)$, por $\tau_{\mathrm{RS}}((W, \partial W, g) ; \rho)$ a $\operatorname{RS}$ torção de $(W, \partial W, g)$. Denotamos por $T_{\text {abs }}((W, g) ; \rho)$ a torção analítica de $(W, g)$, com condições de contorno absolutas em $\partial W$, e por $T_{\text {rel }}((W, g) ; \rho)$ a torção analítica de $(W, g)$, com condições de contorno relativas, ambas com respeito à representação $\rho$ (Capítulo 1). Isto pôsto, obtemos a RS torção do 
cone geométrico sobre as esferas (Seção 4.4) e a torção analítica do disco (Seção 4.5).

De volta às formas espaciais esféricas, apresentamos no Capítulo 3 a construção da região fundamental $\mathcal{F}_{4 t, 4 n-1}$ para a ação do grupo dos quatérnios generalizado $\mathcal{Q}_{4 t}$ sobre a esfera de dimensão ímpar $4 n-1$. Com isso, construímos um complexo de cadeias de modo a calcularmos a R torção (Teorema 4.6) do espaço quociente, chamado de forma espacial esférica quaterniônica, para uma dada representação $\rho$ para seu grupo fundamental. Aproveitamos tal complexo para aplicarmos a teoria da Seção 1.4 e calcularmos também a RS torção (Teorema 4.7).

Observamos que o cálculo da torção analítica das esferas foi feito explicitamente em [30], por meio do uso do espectro do operador Laplaciano. Optamos por calcular a RS torção das esferas (Teorema 4.1), obtendo assim um resultado equivalente, porém de um modo mais simples e sucinto, por meio do Teorema de Cheeger-Müller.

Por fim, apresentamos no Apêndice A a construção dos espaços lenticulares, bem como a classificação destes espaços por meio da R torção. Já no Apêndice B, apresentamos um pouco da geometria utilizada nos cálculos, como por exemplo, Geometria Riemanniana e Teoria de Hodge.

Os Teoremas, Lemas, Exemplos e afins, as Equações e também as Seções, estão numerados de acordo com a numeração dos Capítulos. A diferença é que as equações vêm entre parênteses. Portanto, '(3.3)' refere-se à uma equação enquanto '3.5' refere-se a um resultado ou a uma seção. Por isto, tomamos o cuidado de preceder a numeração com o tipo de objeto a que ela se refere, como por exemplo, 'Proposição 3.5', 'Seção 1.1', etc... Os ítens da bibliografia são, como de costume, exibidos entre colchetes, por exemplo '[31]'. 



\section{CONTEÚDO}

Agradecimentos $\quad$ V

$\begin{array}{lll}\text { Abstract } & \text { VII }\end{array}$

RESUMO IX IX I I I I I I

$\begin{array}{lll}\text { INTRODUÇÃO } & \text { XI }\end{array}$

1 W, R, RS TORÇÕES E TORÇÃO ANALÍTICA 3

1.1 Preliminares e notações . . . . . . . . . . . . . . . . . . . . . 3

1.2 Determinante não comutativo $\ldots \ldots \ldots \ldots \ldots \ldots \ldots$

$1.3 \mathrm{~W}$ e $\mathrm{R}$ torções . . . . . . . . . . . . . . . . . . . . . . . . . . . 10

1.4 RS torção . . . . . . . . . . . . . . . . . . . . . . . . . . . . . . . 12

1.5 Torção analítica . . . . . . . . . . . . . . . . . . . . . . . 16

2 FORMAS ESPACIAIS ESFÉRICAS 19

2.1 Representação de grupos finitos . . . . . . . . . . . . . . . . . . . . 19

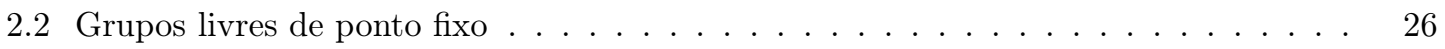

2.3 Classificação das formas espaciais esféricas . . . . . . . . . . . . . 28

3 FORMAS ESPACIAIS ESFÉRICAS QUATERNIÔNICAS 31

3.1 Grupo dos quatérnios generalizado $\mathcal{Q}_{4 t} \ldots \ldots \ldots \ldots \ldots$

3.2 Representações de $\mathcal{Q}_{4 t} \ldots \ldots \ldots \ldots \ldots \ldots \ldots \ldots$

3.3 Região fundamental para a ação de $\mathcal{Q}_{4 t}$ nas esferas . . . . . . . . . . . . . . 34

3.3 .1 Notações . . . . . . . . . . . . . . . . . . . . . . . . . . . . . . . . . 34

$3.3 .2 \quad$ Ação de $\mathcal{Q}_{8}$ em $S^{3} \ldots \ldots \ldots \ldots \ldots \ldots \ldots \ldots$

$3.3 .3 \quad$ Ação de $\mathcal{Q}_{4 t}$ em $S^{3} \ldots \ldots \ldots \ldots \ldots \ldots \ldots \ldots \ldots$

$3.3 .4 \quad$ Ação de $\mathcal{Q}_{16}$ em $S^{7} \ldots \ldots \ldots \ldots \ldots \ldots \ldots$

3.3 .5 Ação de $\mathcal{Q}_{4 t}$ em $S^{4 n-1} \ldots \ldots \ldots \ldots \ldots \ldots \ldots \ldots$

4 CÁlCulo das torÇões $\quad 49$

4.1 Torções das esferas . . . . . . . . . . . . . . . . . . . . . . . . . . . . . . . 49 
4.2 Torções dos espaços lenticulares . . . . . . . . . . . . . . . . . . . . . . 51

4.3 Torções das formas espaciais esféricas quaterniônicas . . . . . . . . . . . . . . . . 52

4.4 RS torção do cone geométrico sobre a esfera . . . . . . . . . . . . . . . . . . 56

4.5 Torção analítica do disco . . . . . . . . . . . . . . . . . . . . . 58

$\begin{array}{lr}\text { A Espaços lenticulares } & 61\end{array}$

A.1 O espaço tridimensional $L_{p, q} \ldots \ldots \ldots \ldots$. . . . . . . . . . . . . . 62

A.2 Estrutura celular e grupos de homologia . . . . . . . . . . . . . . . . . . . 63

A.3 Classificação homotópica . . . . . . . . . . . . . . . . . . . . . 65

$\begin{array}{ll}\text { B GEOMETRIA } & 67\end{array}$

B.1 Tensores e formas diferenciais . . . . . . . . . . . . . . . . . . . 67

B.2 Geometria Riemanniana . . . . . . . . . . . . . . . . . . . . 70

B.3 Teoria de Hodge . . . . . . . . . . . . . . . . . . . . . . . 72

$\begin{array}{ll}\text { Lista DE TABELAS E FIGURAS } & 77\end{array}$

$\begin{array}{lr}\text { LiSTA DE SÍMBOLOS } & 79\end{array}$

REFERÊNCIAS BIBLIOGRÁFICAS $\quad 83$

$\begin{array}{lr}\text { ÍNDICE REMISSIVO } & 87\end{array}$ 


\section{W, R, RS TORÇÕES E TORÇÃO ANALÍTICA}

Neste capítulo, apresentamos inicialmente as definições necessárias para o estudo das torções. Por seguinte, definimos a W torção e a R torção, torções com aspecto mais algébrico. Por fim, concluímos com as definições da RS torção e torção analítica, fazendo uso da geometria da variedade em questão. As referências clássicas são [21, 27].

Convenção: A palavra "módulo" será sempre usada para " $R$-módulo à esquerda finitamente gerado". Também exigiremos o seguinte para o anel $R$ : o módulo livre de 'rank' $r$ sobre $R$ não deve ser isomorfo ao módulo livre de 'rank' $s$, se $r \neq s$.

\subsection{Preliminares e notações}

Nesta seção colocamos os fatos básicos sobre a torção e introduzimos algumas notações. Preferimos definir a torção de Reidemeister ( $\mathrm{R}$ torção) a partir da torção de Whitehead (W torção), mais abstrata. Por um lado, isto torna a apresentação mais geral, e muito mais útil para novas aplicações, por outro lado nos permite descrever de uma maneira concisa as principais propriedades de invariância da $\mathrm{R}$ torção, necessária para nossas aplicações. Pelas mesmas razões, consideramos o caso geral de um espaço não simplesmente conexo. Esta seção é essencialmente baseada em $[9,21,27]$.

Seja $R$ um anel associativo com unidade. O grupo (aditivo) de todas as matrizes $n \times n$ não singulares sobre $R$ será denotado por $G L(n, R)$ (por vezes usaremos uma estrutura de anel). Identificamos cada $M \in G L(n, R)$ com a matriz

$$
\left(\begin{array}{cc}
M & 0 \\
0 & 1
\end{array}\right) \in G L(n+1, R)
$$

e obtemos inclusões $G L(1, R) \subset G L(2, R) \subset \cdots$. A união é chamada grupo linear geral infinito, denotado por $G L(R)$.

Uma matriz é chamada elementar se coincide com a identidade exceto para um único elemento fora da diagonal principal.

1.1 LEMA (J.H.C. WhitehEAD): O subgrupo $E(R) \subset G L(R)$ gerado por todas as matrizes elementares é igual ao subgrupo comutador de $G L(R)$. 
PROVA: Denotemos por $a E_{i j}$ a matriz com $a$ na entrada $(i, j)$ e zero, caso contrário. A igualdade

$$
\left(I+a E_{i j}\right)\left(I+E_{j k}\right)\left(I-a E_{i j}\right)\left(I-E_{j k}\right)=\left(I+a E_{i k}\right),
$$

para $i \neq j \neq k \neq i$, mostra que cada matriz elementar em $G L(n, R)$ é um comutador, para $n \geq 3$ (se $n=2$ basta incluir em dimensão maior).

Reciprocamente, as três igualdades abaixo mostram que cada comutador $X Y X^{-1} Y^{-1} \mathrm{em}$ $G L(n, R)$ pode ser expresso como um produto de matrizes elementares no grupo maior $G L(2 n, R)$.

1. $\left(\begin{array}{cc}X Y X^{-1} Y^{-1} & 0 \\ 0 & I\end{array}\right)=\left(\begin{array}{cc}X & 0 \\ 0 & X^{-1}\end{array}\right)\left(\begin{array}{cc}Y & 0 \\ 0 & Y^{-1}\end{array}\right)\left(\begin{array}{cc}(Y X)^{-1} & 0 \\ 0 & Y X\end{array}\right)$,

2. $\left(\begin{array}{cc}X & 0 \\ 0 & X^{-1}\end{array}\right)=\left(\begin{array}{cc}I & X \\ 0 & I\end{array}\right)\left(\begin{array}{cc}I & 0 \\ I-X^{-1} & I\end{array}\right)\left(\begin{array}{cc}I & -I \\ 0 & I\end{array}\right)\left(\begin{array}{cc}I & 0 \\ I-X & I\end{array}\right)$,

3. $\left(\begin{array}{cc}I & X \\ 0 & I\end{array}\right)=\prod_{i=1}^{n} \prod_{j=n+1}^{2 n}\left(I+x_{i j} E_{i j}\right)$.

Segue que $E(R)$ é um subgrupo normal de $G L(R)$, com grupo quociente comutativo. O quociente será chamado de grupo de Whitehead

$$
K_{1}(R)=G L(R) / E(R)
$$

Geralmente pensaremos em $K_{1}(R)$ como um grupo aditivo.

1.2 OBSERVAÇÃo ([9, Seção 10]): As classes do quociente $G L(R) / E(R)$ são as classes da relação de equivalência: $A \sim B$ se, e somente se, existem matrizes elementares $E_{1}, E_{2} \in E(R)$ tal que $A=E_{1} B E_{2}$.

Se o anel $R$ for comutativo, denotando por $R^{\times}$as unidades de $R$, a função determinante det $: K_{1}(R) \rightarrow R^{\times},[A] \mapsto \operatorname{det}(A)$, é um homomorfismo de grupos bem definido, desde que transformações elementares não modificam o determinante de uma matriz, ou seja, $\operatorname{det} A E=$ $\operatorname{det} A \operatorname{det} E=\operatorname{det} A$, pois $\operatorname{det} E=1$. A imagem está em $R^{\times}$pois as matrizes do grupo linear geral $G L(R)$ são invertíveis. Considerando o grupo linear especial $S L(R)$, consistindo de todas as matrizes em $G L(R)$ com determinante 1, podemos definir o quociente $S L(R) / E(R)$, denotado por $S K_{1}(R)$ e chamado de grupo de Whitehead especial. Notemos a decomposição em soma direta

$$
K_{1}(R) \simeq R^{\times} \oplus S K_{1}(R),
$$

devido à inclusão $R^{\times} \subset G L(1, R) \subset G L(R)$ e à seqüência exata curta

$$
0 \longrightarrow S K_{1}(R) \stackrel{i}{\longrightarrow} K_{1}(R) \stackrel{\operatorname{det}}{\longrightarrow} R^{\times} \longrightarrow 0 \text {. }
$$


1.3 EXEMPLO: Se $\mathbb{F}$ é um corpo então $K_{1}(\mathbb{F}) \simeq \mathbb{F}^{\times}=\mathbb{F}-\{0\}$. Se $\mathbb{F}$ é um anel de divisão, então $K_{1}(\mathbb{F})$ pode ser identificado com o grupo abelianizado $\mathbb{F}^{\times} /\left[\mathbb{F}^{\times}, \mathbb{F}^{\times}\right]$. O homomorfismo natural $G L(n, \mathbb{F}) \rightarrow K_{1}(\mathbb{F})$ é exatamente o determinante de Dieudonné $[1,2,14]$, descrito na Seção 1.2.

1.4 LEMA: Seja $M(n, R)$ o grupo de todas as matrizes $n \times n$ sobre o anel $R$. Então

$$
K_{1}(M(n, R)) \simeq K_{1}(R)
$$

PROVA: Qualquer matriz $k \times k$ com entradas em $M(n, R)$ pode ser pensada como uma matriz $k n \times k n$ com entradas em $R$. Em outras palavras

$$
G L(k, M(n, R)) \simeq G L(k n, R) .
$$

Tomando o limite quando $k \rightarrow \infty$ e então abelianizando obtemos o isomorfismo desejado, ou seja, $K_{1}(M(n, R)) \simeq K_{1}(R)$.

1.5 Definição (Grupo de Whitehead Reduzido): Seja $[-1] \in K_{1}(R)$ o elemento de ordem 2 correspondendo à unidade $(-1) \in G L(1, R) \subset G L(R)$. O quociente

$$
\bar{K}_{1}(R)=K_{1}(R) /\{[1],[-1]\}
$$

será chamado ${ }^{1}$ de grupo de Whitehead reduzido de $R$.

A vantagem de passar para tal quociente é que duas matrizes $A, B$ que diferem somente por uma permutação de colunas (ou linhas) representam o mesmo elemento em $\bar{K}_{1}(R)$. De fato, se $A=B P$ (ou $A=P B$ ) com $P \in G L(n, R)$ obtida permutando as colunas (ou linhas) da identidade por meio de uma permutação $\sigma \in S_{n}$, então $[A]=[B] \in \bar{K}_{1}(R)$, já que $[P]=[\operatorname{sgn}(\sigma)]=[ \pm 1] \in K_{1}(R)$, ou seja, $[P]=[1] \in \bar{K}_{1}(R)$.

Dois exemplos importantes. O grupo de Whitehead do grupo trivial é trivial. De fato, como o anel de grupos inteiro do grupo trivial é $\mathbb{Z}$, toda matriz pode ser escrita como o produto de uma matriz diagonal com um produto de matrizes elementares (ver Lema 1.14). Para os números reais $\mathbb{R}, \bar{K}_{1}(\mathbb{R})$ é isomorfo ao grupo multiplicativo $\mathbb{R}^{+}$dos números reais positivos. Um isomorfismo específico é dado pela correspondência $\left(a_{i j}\right) \mapsto\left|\operatorname{det}\left(a_{i j}\right)\right|$. Para outros exemplos bem mais sofisticados, ver [25].

Se $R$ é comutativo, o homomorfismo determinante det $: K_{1}(R) \rightarrow R^{\times}$definido anteriormente passa para o quociente (também denotado por det) det $: \bar{K}_{1}(R) \rightarrow R^{\times} /\{ \pm 1\}$, ou seja, se $A=$ $\pm B E$ então $\operatorname{det} A= \pm \operatorname{det} B$, pois matrizes elementares possuem determinante 1. Assim sendo, $\operatorname{det}[A]=\operatorname{det}[B] \in R^{\times} /\{ \pm 1\}$.

\footnotetext{
${ }^{1}$ Em alguns casos não comutativos pode acontecer de $[-1]=[1]$ de modo que $\bar{K}_{1}(R)=K_{1}(R)$.
} 
1.6 ExEMPLO: Sejam $G$ um grupo e $\mathbb{R} G$ seu anel de grupos real. Podemos definir uma função determinante em $K_{1}(\mathbb{R} G)$ mesmo se $\mathbb{R} G$ não é abeliano. De fato, seja $\rho: G \rightarrow O(n, \mathbb{R})$ uma representação que se estende a um homomorfismo de anéis $\rho: \mathbb{R} G \rightarrow \mathbb{R} O(n, \mathbb{R})=M(n, \mathbb{R})$. Então, se $A=\left(a_{i j}\right)$ é uma matriz $k \times k$ com entradas em $\mathbb{R} G, \rho(A)=\left(\rho\left(a_{i j}\right)\right)$ é uma matriz $n k \times n k$ com entradas em $\mathbb{R}$ e portanto a função det $: K_{1}(\mathbb{R} G) \rightarrow \mathbb{R}^{\times}$, $\operatorname{det}:[A] \mapsto \operatorname{det}(\rho(A))$, é um homomorfismo bem definido.

1.7 Definição (Grupo de Whitehead de um grupo): Dados um grupo $G$ e um anel $R$, formamos o anel de grupos $R G$. Seja $\varphi: G \rightarrow G L(R G)$ o homomorfismo induzido da inclusão $\varphi: G \rightarrow(R G)^{\times}=G L(1, R G)$. Esta imersão passa para o quociente definindo um homomorfismo $\varphi: G \rightarrow \bar{K}_{1}(R G)$. O quociente

$$
\mathrm{Wh}(R G)=\bar{K}_{1}(R G) / \varphi(G)
$$

é o grupo de Whitehead de $R G$ e a projeção natural é denotada por

$$
w: \bar{K}_{1}(R G) \rightarrow \mathrm{Wh}(R G) .
$$

Em particular, se $R=\mathbb{Z}$ temos o grupo de Whitehead de $G$, Wh $(G)$.

Seja $M$ um $R$-módulo livre e sejam $b=\left(b_{1}, \ldots, b_{k}\right)$ e $c=\left(c_{1}, \ldots, c_{k}\right)$ duas bases distintas para $M$. Tomando $c_{i}=\sum a_{i j} b_{j}$ obtemos uma matriz não singular $(c / b)=\left(a_{i j}\right)$ com entradas em $R$. O elemento correspondente no grupo de Whitehead reduzido $\bar{K}_{1}(R)$ será denotado por $[c / b]$. Se $[c / b]=0$, dizemos que $b$ é equivalente a $c(b \approx c)$. As igualdades $[b / b]=0$ e $[d / c]+[c / b]=[d / b]$ mostram que " $\approx$ " é uma relação de equivalência.

1.8 EXEMPLO: Para um módulo livre de rank 2 , as bases $\left(b_{1}+a b_{2}, b_{2}\right),\left(b_{1}, a b_{1}+b_{2}\right)$ e $\left(b_{2}, b_{1}\right)$ são todas equivalentes a $\left(b_{1}, b_{2}\right)$.

Consideremos uma seqüência exata curta

$$
0 \rightarrow E \rightarrow F \rightarrow G \rightarrow 0
$$

de módulos livres. Dadas bases $e=\left(e_{1}, \ldots, e_{k}\right)$ para $E$ e $g=\left(g_{1}, \ldots, g_{l}\right)$ para $G$, podemos construir uma base $e g$ para $F$ do seguinte modo: levantamos cada $g_{i} \in G$ a um elemento $g_{i}^{\prime} \in F$. Então

$$
e g=\left(e_{1}, \ldots, e_{k}, g_{1}^{\prime}, \ldots, g_{l}^{\prime}\right)
$$

é a base desejada. Claro que esta base eg depende da escolha de $g_{i}^{\prime}$. No entanto, a classe de equivalência de $e g$ depende somente de $e$ e $g$. De fato, se

$$
\overline{e g}=\left(e_{1}, \ldots, e_{k}, g_{1}^{\prime \prime}, \ldots, g_{l}^{\prime \prime}\right)
$$


é obtida com outra escolha $g_{i}^{\prime \prime}$, então $[\overline{e g} / e g]=[e / e]+[g / g]=0$, pois se $\bar{e}$ e $\bar{g}$ são bases alternativas para os módulos $E$ e $G$, respectivamente, então $[\bar{e} \bar{g} / e g]=[\bar{e} / e]+[\bar{g} / g]$, já que

$$
(\bar{e} \bar{g} / e g)=\left(\begin{array}{cc}
(\bar{e} / e) & 0 \\
0 & (\bar{g} / g)
\end{array}\right) .
$$

1.9 OBSERVAÇÃo (Módulo estavelmente livre): A construção acima pode ser feita também para módulos estavelmente livres ao invés de módulos livres. Um módulo $M$ sobre $R$ é estavelmente livre se a soma direta de $M$ com algum módulo livre é livre. Lembramos que todos os módulos aqui são finitamente gerados. Para mais detalhes, ver [21, §4].

\subsection{Determinante não comutativo}

$\mathrm{Na}$ Álgebra, um determinante é uma função que associa a cada matriz $A(n \times n)$ um escalar, $\operatorname{det}(A)$. Fixado um inteiro $n>0$, existe uma única função determinante para as matrizes $n \times n$ sobre um anel comutativo $R$. No entanto, também para anéis não comutativos é possível definirmos a função determinante. Apresentamos aqui um resumo da construção do determinante de Dieudonné e o aplicamos ao anel dos quatérnios H. Para maiores detalhes e para as provas omitidas, sugerimos o bom trabalho de H. Aslaksen [2] e as referências lá citadas ou [1].

Os quatérnios, descoberto por Sir William Rowan Hamilton em 1843, formam uma álgebra associativa não comutativa sobre $\mathbb{R}$,

$$
\mathbb{H}=\{a+b i+c j+d k ; a, b, c, d \in \mathbb{R}\}
$$

onde

$$
i j=k=-j i, \quad j k=i=-k j, \quad k i=j=-i k, \quad i^{2}=j^{2}=k^{2}=-1 .
$$

Podemos escrever $z \in \mathbb{H}$ na forma $z=x+y j$, onde $x, y \in \mathbb{C}$, mas precisamos lembrar que $j y=\bar{y} j$, para $y \in \mathbb{C}$. Note que $\mathbb{H}$ não é uma álgebra sobre $\mathbb{C}$, desde que o centro de $\mathbb{H}$ é somente $\mathbb{R}$. A conjugação em $\mathbb{H}$ é definida por $\overline{a+b i+c j+d k}=a-b i-c j-d k$ e satisfaz $\overline{u v}=\bar{v} \bar{u}$. Os quatérnios da forma $b i+c j+d k$, com $b, c, d \in \mathbb{R}$, serão chamados de quatérnios puros. O seguinte lema nos diz que esta álgebra é isomorfa à uma subálgebra de $M(2, \mathbb{C})$.

1.10 LEMA: Para cada quatérnio $q=a+b i+c j+d k$, definimos $A(q) \in M(2, \mathbb{C})$ por

$$
A(q)=\left(\begin{array}{cc}
a+b i & c+d i \\
-c+d i & a-b i
\end{array}\right) .
$$

Então a aplicação $q \mapsto A(q)$ é injetora e $A\left(q_{1} q_{2}\right)=A\left(q_{1}\right) A\left(q_{2}\right)$.

Definimos a norma (Euclidiana) de um quatérnio por

$$
|q|^{2}=q \bar{q}=a^{2}+b^{2}+c^{2}+d^{2} \quad(q=a+b i+c j+d k) .
$$


Segue que $|q|^{2}=|\bar{q}|^{2}$ e se $|q|^{2} \neq 0$, isto é, $q \neq 0$, então $q\left(\bar{q} /|q|^{2}\right)=1$, de modo que cada quatérnio não nulo possui um inverso multiplicativo $q^{-1}=\bar{q} /|q|^{2}$. Enunciamos então o seguinte:

1.11 LEMA: A álgebra $\mathbb{H}$ dos quatérnios é uma álgebra de divisão, isto é, para cada quatérnio não nulo $q$ existe um inverso (à direita e à esquerda) $q^{-1}$.

De (1.1) e (1.2) é imediato que, para todo quatérnio $q,|q|^{2}=\operatorname{det} A(q)$ e portanto

$$
\left|q_{1} q_{2}\right|^{2}=\operatorname{det} A\left(q_{1} q_{2}\right)=\operatorname{det}\left(A\left(q_{1}\right) A\left(q_{2}\right)\right)=\operatorname{det} A\left(q_{1}\right) \operatorname{det} A\left(q_{2}\right)=\left|q_{1}\right|^{2}\left|q_{2}\right|^{2} .
$$

De (1.3) deduzimos que o conjunto dos quatérnios de norma 1 forma um grupo multiplicativo, denotado por $\mathbb{H}_{1}$. De (1.2) segue que para $q \in \mathbb{H}_{1}, q^{-1}=\bar{q}$. Se pensarmos $\mathbb{H}$ como um espaço 4-dimensional com coordenadas $a, b, c, d$ então $\mathbb{H}_{1}$ é simplesmente a 3-esfera em $\mathbb{R}^{4}$.

Sejam $R$ um anel e $R^{\times}$as unidades de $R$, ou seja, os elementos invertíveis. Se $R$ é um anel de divisão (diferencia-se de um corpo pelo fato de não exigirmos que o produto seja comutativo), então $R^{\times}=R-\{0\}$. Seja $M(n, R)$ o anel das matrizes $n \times n$ com entradas em $R$. Denotaremos o conjunto das matrizes invertíveis de $M(n, R)$ por $G L(n, R)$ e por $E(n, R)$ o subgrupo gerado pelas matrizes elementares $b E_{i j}$, que coincidem com a matriz identidade, exceto pelo elemento $b$ na entrada $(i, j), i \neq j$ (comparar com a Seção 1.1).

Uma função $d: M(n, \mathbb{H}) \rightarrow \mathbb{H}$ é chamada de determinante se satisfaz os seguintes axiomas:

A1. $d(A)=0$ se, e somente se, $A$ é singular.

A2. $d(A B)=d(A) d(B)$, para quaisquer $A, B \in M(n, \mathbb{H})$.

A3. Se $A^{\prime}$ é obtida de $A$ somando um múltiplo à esquerda de uma linha a outra linha ou um múltiplo à direita de uma coluna a outra coluna, então $d\left(A^{\prime}\right)=d(A)$.

Pode-se provar que se $d$ não é nula nem constante igual a 1, então o Axioma 2 implica que $d(A)=0$ para todas as matrizes singulares. Assim, precisamos apenas definir o determinante de matrizes invertíveis. Uma questão natural é saber quando tal determinante existe. Para isso, enunciamos o seguinte teorema.

1.12 TEOREMA: Se $d$ é um determinante, ou seja, $d$ satisfaz os três axiomas acima, então a imagem $d(M(n, \mathbb{H}))$ é um subconjunto comutativo de $\mathbb{H}$.

A prova do Teorema 1.12 é baseada nos dois seguintes lemas. Sem perda de generalidade, podemos supor $n=2$.

1.13 LEMA: Sejam $a \neq 0$ e $d$ um determinante. Então

$$
\left(\begin{array}{cc}
a & 0 \\
0 & a^{-1}
\end{array}\right)=\left(\begin{array}{cc}
1 & 0 \\
-a^{-1} & 1
\end{array}\right)\left(\begin{array}{cc}
1 & a-1 \\
0 & 1
\end{array}\right)\left(\begin{array}{ll}
1 & 0 \\
1 & 1
\end{array}\right)\left(\begin{array}{cc}
1 & a^{-1}-1 \\
0 & 1
\end{array}\right),
$$




$$
d\left(\begin{array}{cc}
a & 0 \\
0 & a^{-1}
\end{array}\right)=1
$$

PROVA: Um cálculo direto prova a igualdade na equação (1.4), cujas matrizes do lado direito são obtidas da matriz identidade através de operações elementares nas colunas, sendo assim aplicadas por $d$ no elemento 1, pelo Axioma 3. Por fim, pelo Axioma 2, segue o resultado.

1.14 LEMA: Toda matriz $A \in G L(n, \mathbb{H})$ pode ser escrita na forma $A=D(x) B$, com $B \in E(n, \mathbb{H})$ e

$$
D(x)=\left(\begin{array}{llll}
1 & & & \\
& \ddots & & \\
& & 1 & \\
& & & x
\end{array}\right) .
$$

PROVA: Como $A$ é invertível, existe ao menos um elemento não nulo na primeira linha, digamos $a_{1 j} \neq 0$. Somando a $j$-ésima coluna, multiplicada por $a_{1 j}^{-1}\left(1-a_{11}\right)$, à direita da primeira coluna, obtemos uma matriz com $a_{11}=1$. Podemos então zerar todos os outros elementos na primeira linha (através de operações nas colunas) e proceder por indução.

Prova do TeOrema 1.12: Definimos $f: \mathbb{H} \rightarrow \mathbb{H}$ por $f(x)=d(D(x))$. Segue do Lema 1.14 que $f(\mathbb{H})=d(M(n, \mathbb{H}))$. Podemos supor $n=2$, por simplicidade. Temos que

$$
d\left(\begin{array}{ll}
x & 0 \\
0 & 1
\end{array}\right)=d\left(\left(\begin{array}{cc}
x & 0 \\
0 & x^{-1}
\end{array}\right)\left(\begin{array}{ll}
1 & 0 \\
0 & x
\end{array}\right)\right)=f(x)
$$

pelo Axioma 2 e Lema 1.13. Mas então

$$
\begin{aligned}
f(x) f(y) & =d\left(\left(\begin{array}{ll}
x & 0 \\
0 & 1
\end{array}\right)\left(\begin{array}{ll}
1 & 0 \\
0 & y
\end{array}\right)\right)=d\left(\begin{array}{ll}
x & 0 \\
0 & y
\end{array}\right) \\
& =d\left(\left(\begin{array}{ll}
1 & 0 \\
0 & y
\end{array}\right)\left(\begin{array}{ll}
x & 0 \\
0 & 1
\end{array}\right)\right)=f(y) f(x)
\end{aligned}
$$

e assim vemos que $f(\mathbb{H})=d(M(n, \mathbb{H}))$ é comutativo.

O Lema 1.1 na página 3 nos diz que $E(n, \mathbb{H})$ é o subgrupo comutador de $G L(n, \mathbb{H})$, ou seja, $E(n, \mathbb{H})=[G L(n, \mathbb{H}), G L(n, \mathbb{H})]$. O Lema 1.14 acima nos diz que é suficiente definirmos o determinante para as matrizes $D(x)$. A preocupação com a unicidade em tal decomposição nos leva à seguinte questão: para quais $x \in \mathbb{H}, D(x) \in E(n, \mathbb{H})$ ? A resposta é dada pelo Lema 1.15 abaixo.

1.15 LEMA ([2, LeMA 7]): O elemento $D(x) \in G L(n, \mathrm{H})$ é um comutador, isto é, $D(x) \in E(n, \mathbb{H})$ se, e somente se, $x \in \mathbb{H}^{\times}$é um comutador. 
Segue que, na decomposição $A=D(x) B$, nem $x$ nem $B$ são únicos, mas a classe lateral $x\left[\mathbb{H}^{\times}, \mathbb{H}^{\times}\right] \in \mathbb{H}^{\times} /\left[\mathbb{H}^{\times}, H^{\times}\right]$é única. Isto foi exatamente o que Jean Dieudonné usou em seu artigo de 1943 [14]. Seu objetivo foi mostrar como o determinante poderia ser expresso em termos da teoria de grupos. Assim, seu principal teorema afirma que, para qualquer anel de divisão $R$, existe um isomorfismo

$$
G L(n, R) /[G L(n, R), G L(n, R)] \rightarrow R^{\times} /\left[R^{\times}, R^{\times}\right] .
$$

Para $R=\mathbb{H}$, isto é imediato dos Lemas 1.14 e 1.15. Definimos então o determinante de Dieudonné por

$$
\operatorname{det} A=\operatorname{det}(D(x) B)=x\left[\mathbb{H}^{\times}, \mathbb{H}^{\times}\right]
$$

bem definido devido ao Lema 1.15, cujo núcleo é $E(n, \mathbb{H})$, coincidindo neste caso com $S L(n, \mathbb{H})$, o grupo linear especial. Se estendermos a definição para $M(n, \mathbb{H})$, o determinante terá valores em $\mathbb{H}^{\times} /\left[\mathbb{H}^{\times}, \mathbb{H}^{\times}\right] \cup\{0\}$. O Lema 8 de $[2]$ prova que $\left[\mathbb{H}^{\times}, \mathbb{H}^{\times}\right] \simeq \mathbb{H}_{1}$. Assim, $\mathbb{H}^{\times} /\left[\mathbb{H}^{\times}, \mathbb{H}^{\times}\right]$é isomorfo ao conjunto dos números reais positivos, via $x\left[\mathbb{H}^{\times}, \mathbb{H}^{\times}\right] \mapsto|x|$.

1.16 O BSERVAÇÃO: Além dos três axiomas, o determinante de Dieudonné possui várias outras propriedades, dentre elas, é invariante pela troca de duas linhas. De fato, trocar as linhas $i$ e $j$ corresponde a multiplicar à esquerda pela matriz $P_{i j}=\left(1 E_{i j}\right)\left(-1 E_{j i}\right)\left(1 E_{i j}\right)$. Porém, $-1 \in \mathbb{H}_{1} \mathrm{e}$ assim det $P_{i j}=1 \mathrm{H}_{1}$. Também, multiplicar por $m$ uma linha à esquerda ou uma coluna à direita, multiplica o determinante por $m \mathrm{H}_{1}$. Este último produto pode ser à direita ou à esquerda, já que $\mathrm{H}^{\times} / \mathbb{H}_{1}$ é comutativo.

Para $n=2$,

$$
\operatorname{det}\left(\begin{array}{cc}
a & b \\
c & d
\end{array}\right)=\operatorname{det}\left(\begin{array}{cc}
a & b \\
0 & d-c a^{-1} b
\end{array}\right)=\left(a d-a c a^{-1} b\right) \mathrm{H}_{1} \quad(a \neq 0)
$$

e

$$
\operatorname{det}\left(\begin{array}{ll}
0 & b \\
c & d
\end{array}\right)=\operatorname{det}\left(\begin{array}{ll}
c & d \\
0 & b
\end{array}\right)=c b \mathrm{H}_{1}=-b c \mathrm{H}_{1} .
$$

Por outro lado, não podemos fatorar um múltiplo à direita de uma linha, pois

$$
\operatorname{det}\left(\begin{array}{cc}
1 & a \\
b & a b
\end{array}\right)=(a b-b a) \mathbb{H}_{1} \quad \text { e } \quad b \mathbb{H}_{1} \operatorname{det}\left(\begin{array}{cc}
1 & a \\
1 & a
\end{array}\right)=0 .
$$

Usaremos tal determinante no Capítulo 4, quando calcularemos a torção das formas espaciais esféricas quaterniônicas.

\subsection{W e $R$ torções}

Seja

$$
C: \quad C_{m} \stackrel{\partial_{m}}{\longrightarrow} C_{m-1} \stackrel{\partial_{m-1}}{\longrightarrow} \cdots \stackrel{\partial_{2}}{\longrightarrow} C_{1} \stackrel{\partial_{1}}{\longrightarrow} C_{0}
$$


um complexo de cadeias de $R$-módulos livres (de dimensão finita). Denotamos por $Z_{q}=\operatorname{ker} \partial_{q}$, por $B_{q}=\operatorname{im} \partial_{q+1}$ e por $H_{q}=Z_{q} / B_{q}$, como usual. Para cada $q$, assumimos que ambos $B_{q}$ and $H_{q}$ são $R$-módulos livres e fixamos bases ${ }^{2} c_{q}$ para $C_{q}$ e $h_{q}$ para $H_{q}$. Escolhemos uma base $b_{q}$ para $B_{q}$. Usando as inclusões $0 \subseteq B_{q} \subseteq Z_{q} \subseteq C_{q}$ e os isomorfismos $Z_{q} / B_{q} \simeq H_{q}$ e $C_{q} / Z_{q} \simeq B_{q-1}$, vemos que as bases $b_{q}, h_{q}, b_{q-1}$ se combinam para produzirem uma nova base $b_{q} h_{q} b_{q-1}$ para $C_{q}$. No entanto, distinguiremos a base $b_{q-1}$ em $C_{q}$ obtida pelo isomorfismo acima denotando-a por $\tilde{b}_{q-1}$. Assim, a nova base para $C_{q}$ é $b_{q} h_{q} \tilde{b}_{q-1}$.

1.17 DeFINIÇÃO (W TORÇÃo dE UM COMPLEXo de CADEIAS): Nas condições acima, a torção de Whitehead do complexo $C$ com relação à base graduada $h=\left\{h_{q}\right\}$ é a classe

$$
\tau_{\mathrm{W}}(C ; h)=\sum_{q=0}^{m}(-1)^{q}\left[b_{q} h_{q} \tilde{b}_{q-1} / c_{q}\right]
$$

em $\bar{K}_{1}(R)$. Tal torção não depende da escolha de $b_{i}$ desde que, escolhendo bases diferentes $b_{i}^{\prime}$, temos

$$
\sum_{q=0}^{m}(-1)^{q}\left[b_{q}^{\prime} h_{q} \tilde{b}_{q-1}^{\prime} / c_{q}\right]=\sum_{q=0}^{m}(-1)^{q}\left(\left[b_{q} h_{q} \tilde{b}_{q-1} / c_{q}\right]+\left[b_{q}^{\prime} / b_{q}\right]+\left[\tilde{b}_{q-1}^{\prime} / \tilde{b}_{q-1}\right]\right)
$$

onde os últimos dois termos se somam a zero. É claro que $\tau_{\mathrm{W}}(C ; h)$ depende de $c_{q}$ e $h_{q}$.

1.18 DEFINIÇÃO (R TORÇão de UM Complexo de CADEIAs): Se o anel $R$ é abeliano, a torção de Reidemeister do complexo $C$ com relação à base graduada $h=\left\{h_{q}\right\}$ é

$$
\tau_{\mathrm{R}}(C ; h)=\prod_{q=0}^{m} \operatorname{det}\left(b_{q} h_{q} \tilde{b}_{q-1} / c_{q}\right)^{(-1)^{q}} \in R^{\times} /\{ \pm 1\}
$$

Seja $(K, L)$ um par de complexos celulares conexos finitos de dimensão $m$ e seja $(\tilde{K}, \tilde{L})$ sua cobertura universal. Identificamos o grupo fundamental de $K$ com o grupo das transformações cobertura de $\tilde{K}$. Seja $C((\tilde{K}, \tilde{L}) ; R)$ o complexo de cadeias de $(\tilde{K}, \tilde{L})$ com coeficientes em $R$. A ação do grupo das transformações cobertura transforma cada grupo $C_{q}((\tilde{K}, \tilde{L}) ; R)$ em um módulo sobre o anel de grupos $R \pi_{1}(K)$ e cada um destes módulos é $R \pi_{1}(K)$-livre e finitamente gerado, pela escolha natural das $q$-células de $K-L$. Desde que $K$ é finito, segue que $C((\tilde{K}, \tilde{L}) ; R)$ é livre e finitamente gerado sobre $R \pi_{1}(K)$. Temos então um complexo de módulos livres finitamente gerados sobre $R \pi_{1}(K)$ que, seguindo a notação canônica, denotaremos por $C\left((\tilde{K}, \tilde{L}) ; R \pi_{1}(K)\right)$.

\footnotetext{
${ }^{2}$ Abusando da notação, diremos que o módulo trivial possui $\emptyset$ como única base.
} 
Qualquer escolha de bases graduadas $c$ para $C\left((\tilde{K}, \tilde{L}) ; R \pi_{1}(K)\right)$ e $h$ para $H\left(C\left((\tilde{K}, \tilde{L}) ; R \pi_{1}(K)\right)\right)$ nos permite definir a torção $\tau_{\mathrm{W}}\left(C\left((\tilde{K}, \tilde{L}) ; R \pi_{1}(K)\right) ; h\right)$ em $\bar{K}_{1}\left(R \pi_{1}(K)\right)$. No entanto, ainda existe alguma ambigüidade devido à arbitrariedade da escolha das células representantes na cobertura $\tilde{K}$, que se projetam sobre as células representantes da base fixada de $C_{q}$. Usando coeficientes inteiros, as diferentes escolhas de células representantes na cobertura originam diferentes torções em $\bar{K}_{1}\left(\mathbb{Z} \pi_{1}(K)\right)$ que se projetam sobre a mesma classe em $\mathrm{Wh}\left(\pi_{1}(K)\right)$.

1.19 Definição (W TORÇão de UM COMPLeXo CelulAR): Nas condições acima, suponhamos que $H_{q}\left(C\left((\tilde{K}, \tilde{L}) ; \mathbb{Z} \pi_{1}(K)\right)\right)$ sejam módulos livres finitamente gerados sobre $\mathbb{Z} \pi_{1}(K)$. A torção de Whitehead de $K$ com relação à base graduada $h$ é a classe

$$
\tau_{\mathrm{W}}((K, L) ; h)=w\left(\tau_{\mathrm{W}}\left(C\left((\tilde{K}, \tilde{L}) ; \mathbb{Z} \pi_{1}(K)\right) ; h\right)\right),
$$

em $\operatorname{Wh}\left(\pi_{1}(K)\right)$. Se $(K, L)$ é a decomposição celular (ou simplicial) de um espaço $(X, A)$, a torção de Whitehead de $(X, A)$ é definida analogamente e denotada por $\tau_{\mathrm{W}}((X, A) ; h)$.

Foi provado em [21] que $\tau_{\mathrm{W}}((X, A) ; h)$ não depende da decomposição $K$.

Se $\varphi: R \rightarrow R^{\prime}$ é um homomorfismo de anéis, podemos formar um novo complexo $R^{\prime} \otimes_{R} C_{q}$, usando o homomorfismo $\varphi$ para tornar $R^{\prime}$ um $R$-módulo à direita. Então, $\tau_{\mathrm{W}}\left(R^{\prime} \otimes_{R} C ; h^{\prime}\right)=$ $\varphi_{*} \tau_{\mathrm{W}}(C ; h)$. Tal construção é usada para definirmos a torção com relação à uma representação do grupo fundamental. Seja $\rho: \pi_{1}(X) \rightarrow G$ uma representação do grupo fundamental em algum grupo $G$. Então, $\rho$ se estende a um único homomorfismo de anéis de $\mathbb{Z} \pi_{1}(X)$ para $\mathbb{Z} G$ e assim formamos o complexo

$$
C_{q}\left((X, A) ;\left(\mathbb{Z} \pi_{1}(X)\right)_{\rho}\right)=\mathbb{Z} G \otimes_{\rho} C_{q}\left((\tilde{X}, \tilde{A}) ; \mathbb{Z} \pi_{1}(X)\right)
$$

de $\mathbb{Z} G$-módulos livres finitamente gerados. A torção de Whitehead de $(X, A)$ com relação à representação $\rho$ (e base graduada $h$ ) é definida por

$$
\tau_{\mathrm{W}}((X, A) ; h, \rho)=w\left(\tau_{\mathrm{W}}\left(C\left((X, A) ;\left(\mathbb{Z} \pi_{1}(X)_{\rho}\right)\right) ; h\right)\right),
$$

$\mathrm{em} \operatorname{Wh}(G)$.

\subsection{RS torção}

Seja $W$ uma variedade Riemanniana orientável e conexa de dimensão $m$, com métrica $g$, possivelmente com bordo $\partial W$. Então, todas as hipóteses anteriores são satisfeitas e podemos definir uma $\mathrm{R}$ torção absoluta $\tau_{\mathrm{R}}((W, \emptyset) ; h, \rho)$ e uma $\mathrm{R}$ torção relativa $\tau_{\mathrm{R}}((W, \partial W) ; h, \rho)$, para cada representação $\rho$ do grupo fundamental e para cada base graduada $h$ fixada, para a homologia de $(W, \emptyset)$ ou para a homologia relativa de $(W, \partial W)$, respectivamente. Neste contexto, Ray e Singer [27] sugerem um invariante geométrico natural, fixando uma base apropriada $h$, fazendo uso da estrutura geométrica, como segue. 
Seja $K$ uma triangulação suave de $W$. Seja $E_{\rho} \rightarrow W$ o fibrado vetorial real associado à representação $\rho: \pi_{1}(K) \rightarrow O(k, \mathbb{R})$, isto é, $E_{\rho}$ é o fibrado com grupo estrutural $\rho\left(\pi_{1}(K)\right)$, construído do seguinte modo: desde que a cobertura universal $\tilde{K} \rightarrow K$ é um fibrado com fibra $\pi_{1}(K)$, podemos simplesmente mudar a fibra tomando o produto tensorial $\tilde{K} \otimes_{\rho} \mathbb{R}^{k}$, obtendo o fibrado $E_{\rho}$. Seja $\Omega^{*}\left(W, E_{\rho}\right)$ o espaço linear graduado das formas diferenciais em $W$ com valores em $E_{\rho}$ $[4, \S 7]$, isto é, $\Omega^{q}\left(W, E_{\rho}\right)$ é o espaço das seções dos fibrados $\Omega^{q}(W) \otimes_{\rho} \mathbb{R}^{k}$, onde $\Omega^{q}(W)$ é o espaço das $q$-formas diferenciais sobre $W$ (ver Seção B.1). Uma base para $\Omega^{q}\left(W, E_{\rho}\right)$ é da forma $\left\{C^{\infty}(W) \otimes d x_{I} \otimes_{\rho} e_{i}\right\}$, onde $I=\left(i_{1}, \ldots, i_{q}\right)$ é um multi-índice, $\left\{x_{j}\right\}$ são coordenadas em $W$ e $\left\{e_{i}\right\}$ é uma base para $\mathbb{R}^{k}$.

A derivada exterior em $W$ define uma derivada exterior $d: \Omega^{q}\left(W, E_{\rho}\right) \rightarrow \Omega^{q+1}\left(W, E_{\rho}\right)$ por $d\left(\omega \otimes_{\rho} v\right)=d \omega \otimes_{\rho} v$. A métrica $g$ define o operador $\star$ de Hodge em $W$ e portanto em $\Omega^{q}\left(W, E_{\rho}\right)$, $\star: \Omega^{q}\left(W, E_{\rho}\right) \rightarrow \Omega^{m-q}\left(W, E_{\rho}\right)$. Usando o produto interno em $E_{\rho}$, define também um produto interno em $\Omega^{q}\left(W, E_{\rho}\right)$, como segue: dadas as formas

$$
\omega=\sum_{I, j} \omega_{I, j}(x) d x_{I} \otimes e_{j}, \quad \quad \eta=\sum_{I^{\prime}, j^{\prime}} \eta_{I^{\prime}, j^{\prime}}(x) d x_{I^{\prime}} \otimes e_{j^{\prime}},
$$

o produto interno entre elas é dado por

$$
(\omega, \eta)=\sum_{I, I^{\prime}, j, j^{\prime}} \int_{W} \omega_{I, j}(x) \omega_{I^{\prime}, j^{\prime}}(x) d x_{I} \wedge \star d x_{I^{\prime}}\left(e_{j}, e_{j^{\prime}}\right),
$$

onde $(v, w)$ é o produto interno em $\mathbb{R}^{k}$ e $\wedge$ é o produto exterior em $\Omega^{*}(W)$.

Lembramos que existe uma decomposição natural de $\Omega^{*}(W)$ como soma direta de fibrados vetoriais, $\Omega^{*}(\partial W) \oplus N^{*} W$, onde $N^{*} W$ é o dual do fibrado normal (no bordo). Mais precisamente, seja $\partial_{r}$ o vetor normal unitário no bordo "apontando para dentro" e $d r$ a 1-forma correspondente. Próximo ao bordo, temos a decomposição $C(\partial W)=\partial W \times[0, \epsilon)$ e se $y$ é um sistema de coordenadas locais no bordo $\partial W$, então $x=(y, r)$ é um sistema de coordenadas locais em $C(\partial W)$. As formas diferenciais próximas ao bordo de $W$ decompõem-se como $\omega=\omega_{\text {tan }}+\omega_{\text {norm }}$, onde $\omega_{\text {tan }}$ está em $\Omega^{*}(W)$ e $\omega_{\text {norm }}$ é a projeção ortogonal no subespaço gerado por $d r$. Podemos então decompor uma forma $\omega \in \Omega^{*}(W)$ em $\omega=\omega_{1}+\omega_{2} \wedge d r, \omega_{j} \in C^{\infty}(W) \otimes \Omega^{*}(\partial W)$, com $\star \omega_{2}=d r \wedge \star \omega$.

Definimos condições de contorno absolutas e relativas por

$$
B_{\mathrm{abs}}(\omega)=\omega_{\mathrm{norm} \mid \partial W}=\omega_{2 \mid \partial W}=0, \quad B_{\mathrm{rel}}(\omega)=\omega_{\mathrm{tan} \mid \partial W}=\omega_{1 \mid \partial W}=0
$$

Seja $\mathcal{B}(\omega)=B(\omega) \oplus B\left(\left(d+d^{\dagger}\right)(\omega)\right)$, para $B \in\left\{B_{\text {abs }}, B_{\text {rel }}\right\}$. Assim, o operador $\Delta=\left(d+d^{\dagger}\right)^{2}$, com condições de contorno $\mathcal{B}(\omega)=0$, é auto-adjunto [16, Lema 2.7.2(a)]. Note que

$$
\mathcal{B}_{\text {abs }}(\omega)=0 \Longleftrightarrow\left\{\begin{array}{l}
\omega_{\text {norm } \mid \partial W}=0 \\
(d \omega)_{\text {norm } \mid \partial W}=0
\end{array} \quad \text { e } \quad \mathcal{B}_{\text {rel }}(\omega)=0 \Longleftrightarrow\left\{\begin{array}{l}
\omega_{\tan \mid \partial W}=0 \\
\left(d^{\dagger} \omega\right)_{\tan \mid \partial W}=0
\end{array}\right.\right.
$$


pois $\left(d+d^{\dagger}\right)(\omega)_{\text {norm }}=d(\omega)_{\text {norm }}$ e $\left(d+d^{\dagger}\right)(\omega)_{\tan }=d^{\dagger}(\omega)_{\tan }$.

Sejam

$$
\begin{aligned}
\mathcal{H}^{q}\left(W, E_{\rho}\right) & =\left\{\omega \in \Omega^{q}\left(W, E_{\rho}\right) \mid \Delta^{q} \omega=0\right\}, \\
\mathcal{H}_{\mathrm{abs}}^{q}\left(W, E_{\rho}\right) & =\left\{\omega \in \Omega^{q}\left(W, E_{\rho}\right) \mid \Delta^{q} \omega=0, B_{\mathrm{abs}}(\omega)=0\right\}, \\
\mathcal{H}_{\mathrm{rel}}^{q}\left(W, E_{\rho}\right) & =\left\{\omega \in \Omega^{q}\left(W, E_{\rho}\right) \mid \Delta^{q} \omega=0, B_{\mathrm{rel}}(\omega)=0\right\},
\end{aligned}
$$

espaços das formas harmônicas (com condições de contorno nos dois últimos).

Temos então as seguintes aplicações de de Rham $\mathcal{A}^{q}$, que induzem isomorfismo em cohomologia,

$$
\mathcal{A}_{\mathrm{abs}}^{q}: \mathcal{H}_{\mathrm{abs}}^{q}\left(W, E_{\rho}\right) \rightarrow C^{q}\left(W ; E_{\rho}\right), \quad \mathcal{A}_{\mathrm{rel}}^{q}: \mathcal{H}_{\mathrm{rel}}^{q}\left(W, E_{\rho}\right) \rightarrow C^{q}\left((W, \partial W) ; E_{\rho}\right),
$$

com

$$
\mathcal{A}_{\text {abs }}^{q}(\omega)\left(c \otimes_{\rho} v\right)=\mathcal{A}_{\text {rel }}^{q}(\omega)\left(c \otimes_{\rho} v\right)=\int_{c}(\omega, v),
$$

onde $c \otimes_{\rho} v$ pertence a $C^{q}\left(W ; E_{\rho}\right)$ no primeiro caso e a $C^{q}\left((W, \partial W) ; E_{\rho}\right)$ no segundo caso, e $c$ está identificada com a $q$-célula (simplicial ou celular) que representa.

Seja $K$ (resp. $L)$ uma decomposição celular ou simplicial de $W($ resp. $\partial W)$. Seja $\operatorname{sd}(K)$ a primeira subdivisão baricêntrica de $K$. Seja $\hat{K}$ o complexo bloco dual de $K$. Para cada $q$-célula (simplexo) $c$ em $K$, seja $\hat{c}$ o $(m-q)$-bloco dual de $c$ (ver [23, §15]). Se tomarmos $q$-células $c$ em $K-L$, então a coleção de células $\hat{c}$ é uma base para o grupo de $(m-q)$-cadeias relativas $C_{m-q}(\hat{K} ; \mathbb{Z})$. Portanto, a bijeção $c \leftrightarrow \hat{c}$ induz uma bijeção entre as bases de $C_{q}(K, L ; \mathbb{Z})$ e $C_{m-q}(\hat{K} ; \mathbb{Z})$, e portanto um isomorfismo comutando com os operadores bordo,

$$
\varphi_{q}: C_{q}(K, L ; \mathbb{Z}) \rightarrow C_{m-q}(\hat{K}-\hat{L} ; \mathbb{Z}), \quad \varphi_{q}(c)=\hat{c} .
$$

Note que a imagem de $\varphi_{q}$ está em $\hat{K}-\hat{L}$, desde que $\hat{c}$ é disjunto de $\hat{L}$, se $c \in K-L$. A Figura 1.1 exemplifica a bijeção acima, para o par $(K, L)=\left(S^{1}, \emptyset\right)$.
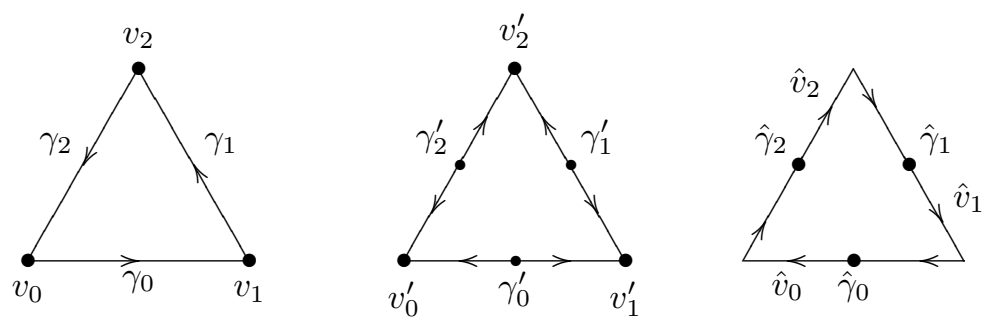

Figura 1.1: Complexos $K, K^{\prime}=\operatorname{sd}(K)$ e $\hat{K}=\operatorname{dual}(K)$.

Considerando $c^{*}$ a cocadeia dual da cadeia $c$, de mesma dimensão, temos o isomorfismo de Lefschetz-Poincaré

$$
\mathcal{P}_{q}: C_{q}(K, L ; \mathbb{Z}) \rightarrow C^{m-q}(\hat{K}-\hat{L} ; \mathbb{Z}), \quad \mathcal{P}_{q}(c)=(\hat{c})^{*},
$$


induzindo um isomorfismo $\mathcal{P}_{q}: H_{q}(K, L ; \mathbb{Z}) \rightarrow H^{m-q}(\hat{K}-\hat{L} ; \mathbb{Z})$.

Podemos também construir uma aplicação $\mathcal{A}^{q}: \Omega^{q}\left(W, E_{\rho}\right) \rightarrow C^{q}(\hat{K}-\hat{L} ; \mathbb{Z})$, com

$$
\mathcal{A}^{q}(\omega): C_{q}(\hat{K}-\hat{L} ; \mathbb{Z}) \rightarrow \mathbb{R}, \quad \mathcal{A}^{q}(\omega)(\hat{c})=\int_{\hat{c}} \omega
$$

induzindo nas formas harmônicas um isomorfismo $\mathcal{A}^{q}: \mathcal{H}^{q}\left(W, E_{\rho}\right) \rightarrow H^{q}(\hat{K}-\hat{L} ; \mathbb{Z})$.

Seguindo Ray e Singer [27], introduzimos as aplicações de de Rham $\mathcal{A}_{q}$ pela composição

$$
\mathcal{H}^{q}\left(W, E_{\rho}\right) \stackrel{\star_{q}}{\longrightarrow} \mathcal{H}^{m-q}\left(W, E_{\rho}\right) \stackrel{\mathcal{A}^{m-q}}{\longrightarrow} H^{m-q}(\hat{K}-\hat{L} ; \mathbb{Z}) \stackrel{\epsilon(q) \mathcal{P}_{q}^{-1}}{\longrightarrow} H_{q}(K, L ; \mathbb{Z}),
$$

ou seja, $\mathcal{A}_{q}=\epsilon(q) \mathcal{P}_{q}^{-1} \mathcal{A}^{m-q} \star_{q}$, com $\epsilon(q)=(-1)^{q(m-1)}$. Para condições de contorno relativas e absolutas, temos explicitamente

$$
\mathcal{A}_{q}^{\mathrm{rel}}: \mathcal{H}_{\mathrm{rel}}^{q}\left(W, E_{\rho}\right) \rightarrow H_{q}(K, L ; \mathbb{Z}), \quad \quad \mathcal{A}_{q}^{\mathrm{abs}}: \mathcal{H}_{\mathrm{abs}}^{q}\left(W, E_{\rho}\right) \rightarrow H_{q}(K ; \mathbb{Z})
$$

ambas definidas por

$$
\mathcal{A}_{q}^{\mathrm{rel}}(\omega)=\mathcal{A}_{q}^{\mathrm{abs}}(\omega)=(-1)^{q(m-1)} \sum_{j, i}\left(\int_{\hat{c}_{q, j}}\left(\star \omega, e_{i}\right)\right) c_{q, j} \otimes_{\rho} e_{i},
$$

onde a soma é sobre todos os $q$-simplexos $c_{q, j}$ de $K$ no primeiro caso, porém sobre todos os $q$ simplexos $c_{q, j}$ de $K-L$ no segundo caso.

1.20 DEFINIÇÃO (RS TORÇÃo): Seja $a$ uma base ortonormal graduada para o espaço das formas harmônicas em $\Omega^{*}(W) \otimes_{\rho} \mathbb{R}^{k}$. O número real positivo

$$
\tau_{\mathrm{RS}}((W, g) ; \rho)=\tau_{\mathrm{R}}\left(W ; \mathcal{A}^{\mathrm{abs}}(a), \rho\right)
$$

é chamado de $R S$ torção de $(W, g)$ com relação à representação $\rho$ e condições de contorno absolutas, e

$$
\tau_{\mathrm{RS}}((W, \partial W, g) ; \rho)=\tau_{\mathrm{R}}\left((W, \partial W) ; \mathcal{A}^{\mathrm{rel}}(a), \rho\right)
$$

é chamado de $R S$ torção de $(W, \partial W, g)$ com relação à representação $\rho$ e condições de contorno relativas.

1.21 OBSERVAÇÃo: Ambas $\tau_{\mathrm{RS}}((W, g) ; \rho)$ e $\tau_{\mathrm{RS}}((W, \partial W, g) ; \rho)$ não dependem da escolha da base ortonormal $a$, desde que uma mudança de base, neste caso, é dada por uma matriz ortogonal. Também, é independente da escolha dos representantes dos simplexos de $K$ em $\tilde{K}$, bem como da triangulação $K$ para $W$. [27, Proposição 3.7]. 
Voltando para a construção anterior, obtemos que a fórmula na equação (1.7) na presente situação nos dá a seguinte fórmula para a RS torção (onde $N$ pode ser $\partial W$ ou o conjunto vazio e $\mathcal{A}_{q}$ pode ser absoluta ou relativa, respectivamente)

$$
\tau_{\mathrm{RS}}((W, N, g) ; \rho)=\prod_{q=0}^{m}\left|\operatorname{det} \rho\left(b_{q} \mathcal{A}_{q}\left(a_{q}\right) \tilde{b}_{q-1} / c_{q}\right)\right|^{(-1)^{q}},
$$

onde det é o determinante usual, no caso acima, da matriz mudança de base no complexo de espaços vetoriais reais $C\left((W, N) ; E_{\rho}\right)$ :

$$
C_{m} \otimes_{\rho} \mathbb{R}^{k} \stackrel{\partial_{m} \otimes_{\rho} 1}{\longrightarrow} \cdots \stackrel{\partial_{2} \otimes_{\rho} 1}{\longrightarrow} C_{1} \otimes_{\rho} \mathbb{R}^{k} \stackrel{\partial_{1} \otimes_{\rho} 1}{\longrightarrow} C_{0} \otimes_{\rho} \mathbb{R}^{k}
$$

\subsection{Torção analítica}

Concluímos o capítulo com a definição da torção analítica. Primeiro, assumimos que $W$ não possui bordo. Com o produto interno definido na equação (1.8), $\Omega^{*}\left(W, E_{\rho}\right)$ é um espaço de Hilbert. Seja $d^{\dagger}=(-1)^{m(q+1)+1} \star d \star$ o adjunto de $d$. Como em (B.4), o Laplaciano $\Delta=\left(d+d^{\dagger}\right)^{2}$ é um operador simétrico positivo definido em $\Omega^{*}\left(W, E_{\rho}\right)$, com espectro $\operatorname{Sp} \Delta$ puramente pontual. Seja $\Delta^{q}$ a restrição de $\Delta$ a $\Omega^{q}\left(W, E_{\rho}\right)$. Então definimos a função zeta de $\Delta^{q}$ pela série

$$
\zeta\left(s, \Delta^{q}\right)=\sum_{\lambda \in \mathrm{Sp}_{+} \Delta^{q}} \lambda^{-s},
$$

para $\operatorname{Re}(s)>\frac{m}{2}$, onde $\mathrm{Sp}_{+}$denota a parte positiva do espectro. A série acima converge uniformemente para $\operatorname{Re}(s)>\frac{m}{2}$ e se estende a uma função meromorfa analítica em $s=0$.

1.22 DefiniçÃo (Torção Analítica): Definimos a torção analítica $T$ de $(W, g)$ por

$$
\log T((W, g) ; \rho)=\frac{1}{2} \sum_{q=1}^{m}(-1)^{q} q \zeta^{\prime}\left(0, \Delta^{q}\right) .
$$

Se $W$ possui bordo, denotamos por $T_{\text {abs }}((W, g) ; \rho)$ (resp. $\left.T_{\text {rel }}((W, g) ; \rho)\right)$ o número definido pela equação (1.12) com $\Delta$ satisfazendo condições de contorno absolutas (resp. relativas).

Enunciamos duas propriedades da torção analítica, análogas às propriedades conhecidas da $\mathrm{R}$ torção. As provas serão omitidas e podem ser encontradas em [27, §2].

1.23 TEOREMA: Seja $W$ uma variedade compacta orientável sem bordo, de dimensão par. Então $\log T((W, g) ; \rho)=0$. O mesmo vale para $\tau_{\mathrm{R}}(W)$ (ver $\left.[20]\right)$.

1.24 TEOREMA: Sejam $W_{1}$ e $W_{2}$ variedades compactas orientáveis sem bordo, com $W_{2}$ simplesmente conexa. A torção analítica do produto $W_{1} \times W_{2}$ é dada por

$$
\log T\left(W_{1} \times W_{2}\right)=\chi\left(W_{2}\right) \log T\left(W_{1}\right),
$$


onde $\chi\left(W_{2}\right)$ é a característica de Euler de $W_{2}$. O resultado correspondente para a $\mathrm{R}$ torção encontra-se em [17].

Em [30] foi calculada a torção analítica da esfera $S^{2 m-1}$, ou seja, $T\left(S^{2 m-1}\right)=\frac{2 \pi^{m}}{(m-1) !}$. Ressaltamos que o método utilizado é puramente analítico e faz uso dos autovalores do Laplaciano. No entanto, apresentamos na Seção 4.1 o mesmo resultado, porém com a abordagem descrita até então, utilizando a RS torção. 



\section{FORMAS ESPACIAIS ESFÉRICAS}

Neste capítulo apresentamos as definições básicas e alguns resultados que nos fornecerão os possíveis grupos ocorrendo na construção das formas espaciais esféricas. Daremos maior atenção a um grupo em particular, o grupo dos quatérnios generalizado, estudado com mais detalhes no Capítulo 3. A referência aqui utilizada é [31, Parte III].

\subsection{Representação de grupos finitos}

Sejam $G$ um grupo e $V$ um espaço vetorial sobre um corpo $\mathbb{F}$. Uma representação de $G$ em $V$ é um homomorfismo $\pi$ de $G$ no grupo das transformações lineares invertíveis de $V$. $\pi$ é uma representação sobre $\mathbb{F}$ e $V$ é chamado o espaço representação de $\pi$. Se $V$ é de dimensão finita (e neste caso dizemos que $\pi$ é de dimensão finita), então a dimensão de $V$ é chamada de grau de $\pi$. Considerando $V^{*}$ o espaço dual de $V$, temos uma representação $\pi^{*}$ de $G$ em $V^{*}$ induzida por $\pi$ definida por $\left(\pi^{*}(g)\left(v^{*}\right)\right)(v)=v^{*}\left(\pi\left(g^{-1}\right)(v)\right)$. Assim, $\pi^{*}$ é a representação dual de $\pi$. Se $V^{* *}=V$ (por exemplo, se $V$ é de dimensão finita) então $\pi^{* *}=\pi$. $\pi$ é chamada de 'faithful' se é injetora. Se $\psi$ é uma representação de $G$ em um espaço vetorial $W$ sobre o mesmo corpo $\mathbb{F}$, então $\pi$ e $\psi$ são equivalentes se existe um isomorfismo $f: V \rightarrow W$ tal que $f \pi(g)=\psi(g) f$ para todo $g \in G$. O caractere de uma representação de dimensão finita $\pi$ é a função $\chi_{\pi}: G \rightarrow \mathbb{F}$ definida por $\chi_{\pi}(g)=\operatorname{tr}(\pi(g))$. Observe que representações equivalentes de dimensão finita possuem o mesmo caractere, pois $\operatorname{tr}(\pi(g))=\operatorname{tr}\left(f^{-1} \psi(g) f\right)=\operatorname{tr}\left(\psi(g) f f^{-1}\right)=\operatorname{tr}(\psi(g))$.

Se $U \subseteq V$ é um subespaço $\pi(G)$-invariante, então $\pi^{\prime}(g): u \mapsto \pi(g) u$ define uma representação $\pi^{\prime}: G \rightarrow U$. Representações $\pi^{\prime}$ desta forma são chamadas de sub-representações de $\pi$. Uma sub-representação $\pi^{\prime}$ em $U$ é própria se $U$ é um subespaço próprio de $V$, isto é, se $0 \subsetneq U \subsetneq V$. $\pi$ é chamada irredutível se não possui sub-representações próprias. Se $\left\{\pi_{i}\right\}$ são representações em espaços vetoriais $\left\{V_{i}\right\}$ sobre $\mathbb{F}$, então a soma direta $\sum \pi_{i}$ é a representação na soma direta $\sum V_{i}$ dada por $\left(\sum \pi_{i}\right)(g): \sum v_{i} \rightarrow \sum \pi_{i}(g) v_{i}, v_{i} \in V_{i}$. $\pi$ é totalmente irredutível se é equivalente a uma soma direta de representações irredutíveis. Se $\pi$ e $\psi$ são representações dos grupos $A$ e $B$ nos espaços $V$ e $W$, então o produto tensorial $\pi \otimes \psi$ é a representação de $A \times B$ em $V \otimes W$ dada por

$$
(\pi \otimes \psi)(a, b): v \otimes w \mapsto \pi(a)(v) \otimes \psi(b)(w)
$$


Se $\pi(a)$ e $\psi(b)$ possuem autovalores $\left\{\lambda_{i}\right\}$ e $\left\{\mu_{k}\right\}$, respectivamente, então $\left\{\lambda_{i} \mu_{k}\right\}$ são os autovalores de $(\pi \otimes \psi)(a, b)$.

Sejam $\pi$ e $\psi$ representações de $G$ sobre $\mathbb{F}$. Se $V$ e $W$ são seus espaços representações, então $\mathbf{I}(\pi, \psi)$ denota o conjunto de todas as transformações lineares $f: V \rightarrow W$ tais que $f \pi(g)=\psi(g) f$ para todo $g \in G$. Os elementos de $\mathbf{I}(\pi, \psi)$ são chamados de operadores 'intertwining' de $\pi$ e $\psi$. Note que $\pi$ é equivalente a $\psi$ se, e somente se, algum operador 'intertwining' é um isomorfismo. Se $G$ é abeliano então $\pi(G) \subset \mathbf{I}(\pi, \pi)$. De fato, sejam $g, g_{0} \in G$ e $\pi(g): V \rightarrow V$. Assim, $\pi\left(g_{0}\right) \pi(g)=\pi\left(g_{0} g\right)=\pi\left(g g_{0}\right)=\pi(g) \pi\left(g_{0}\right)$ e então $\pi(g) \in \mathbf{I}(\pi, \pi)$.

2.1 OBSERVAÇÃo ([31, 4.2.8]): Duas representações de $G$ são equivalente se, e somente se, possuem o mesmo caractere.

Seja $\sigma$ uma representação irredutível de $G$ em um espaço vetorial de dimensão finita $V$ sobre KK. A extensão escalar $V_{\mathrm{F}}=V \otimes_{\mathbb{K}} \mathbb{F}$ é um espaço vetorial de mesma dimensão sobre $\mathbb{F}$ que pode ser criado escolhendo uma base $\left\{v_{i}\right\}$ de $V$ sobre $\mathbb{K}$ e definindo $V_{\mathbb{F}}=\left\{\sum a_{i} v_{i}, a_{i} \in \mathbb{F}\right\}$. Agora $\sigma(G)$ é também um conjunto de transformações $\mathbb{F}$-lineares de $V_{\mathbb{F}}$, assim $\sigma$ nos dá uma representação $\sigma_{\mathbb{F}}$ de $G$ em $V_{\mathrm{F}}$. $\sigma$ é chamada absolutamente irredutivel se $\sigma_{\mathrm{F}}$ é irredutível.

Consideremos agora um grupo finito $G$ de ordem $N$ e representações de $G$ sobre o corpo dos números reais $\mathbb{R}$ ou sobre o corpo do números complexos $\mathbb{C}$. Uma representação sobre $\mathbb{R}$ (resp. sobre $\mathbb{C}$ ) é chamada representação real (resp. representação complexa). Se uma representação complexa $\pi$ é equivalente a $\sigma_{\mathbb{C}}$ para alguma representação real $\sigma$, então cometeremos um abuso de linguagem e diremos que $\pi$ é equivalente a uma representação real.

As isometrias lineares $f: V \rightarrow V$ formam um grupo, chamado grupo ortogonal de $V$ no caso real e grupo unitário de $V$ no caso complexo. Se $V$ é real (resp. complexo), então uma representação de $G$ em $V$ é chamada representação ortogonal (resp. representação unitária) se sua imagem está contida no grupo ortogonal (resp. unitário) de $V$. Se $V$ e $W$ são reais (resp. complexos) e se $\sigma$ e $\tau$ são representações de $G$ em $V$ e $W$, então $\sigma$ e $\tau$ são ortogonalmente equivalentes (resp. unitariamente equivalentes) se existe uma isometria linear $f: V \rightarrow W$ tal que $f \sigma(g)=\tau(g) f$, para todo $g \in G$, e neste caso, é claro, $\sigma$ e $\tau$ são equivalentes.

2.2 LeMA ([31, Lema 4.7.1]): Toda representação real (resp. complexa) de $G$ é equivalente a uma representação ortogonal (resp. unitária). Duas representações ortogonais (resp. unitárias) de $G$ são equivalentes se, e somente se, são ortogonalmente (resp. unitariamente) equivalentes. Se $\sigma$ e $\tau$ são representações ortogonais (resp. unitárias) 'faithful' de $G$ em $V$ e $W$, então existe uma isometria linear $f: V \rightarrow W$ tal que $\tau(G)=f \sigma(G) f^{-1}$ se, e somente se, existe um automorfismo $\alpha$ de $G$ tal que $\tau \alpha$ é equivalente a $\sigma$. 
2.3 LEMA ([31, LeMA 4.7.2]): Seja $\pi$ uma representação complexa de $G$. Se $\left\{v_{i}\right\}$ é uma base do espaço representação $V$ e se $\pi(g)$ tem matriz $\left(\pi(g)_{i j}\right)$ relativa a $\left\{v_{i}\right\}$, então seja $\bar{\pi}(g)$ a transformação linear de $V$ cuja matriz relativa a $\left\{v_{i}\right\}$ é a matriz complexa conjugada $\left(\overline{\pi(g)_{i j}}\right)$ de $\left(\pi(g)_{i j}\right)$. Então $\bar{\pi}$ é uma representação de $G$ em $V$ que é equivalente à dual $\pi^{*}$ de $\pi$. $\bar{\pi}$ é chamada de conjugada de $\pi$.

Prova: Se mudamos a base de $V$ por uma matriz $m=\left(m_{i j}\right)$, então o elemento $\pi(g)_{i j}$ é trocado por $\left(m \pi(g) m^{-1}\right)_{i j}$ e $\bar{\pi}(g)_{i j}$ por $\left(\bar{m} \bar{\pi}(g) \bar{m}^{-1}\right)_{i j}$. Assim, $\bar{\pi}$ é definida (a menos de equivalência) sem menção à base $\left\{v_{i}\right\}$. Podemos então supor que $\pi$ é unitária e que $\left\{v_{i}\right\}$ é ortonormal. Se $\left\{v^{i}\right\}$ é a base de $V^{*}$ dual de $\left\{v_{i}\right\}$ e $\pi^{*}(g)$ tem matriz $\left(\pi^{*}(g)_{i j}\right)$ nesta base, então

$$
\pi^{*}(g)_{i j}=\left(\pi^{*}(g)\left(v^{i}\right)\right)\left(v_{j}\right)=v^{i}\left(\pi\left(g^{-1}\right)\left(v_{j}\right)\right)=v^{i}\left(\bar{\pi}(g)^{t}\left(v_{j}\right)\right)=\bar{\pi}(g)_{i j} .
$$

2.4 Definição (Grupo livre de ponto fixo): Se $\pi$ é uma representação de um grupo $G$ e se $1 \neq g \in G$ implica que $\pi(g)$ não tem +1 como autovalor, então $\pi$ é livre de ponto fixo. Um grupo livre de ponto fixo é um grupo finito abstrato que possui uma representação livre de ponto fixo.

2.5 OBSERVAÇÃO: Sejam $G$ um grupo e $X$ um subconjunto compacto de $\mathbb{R}^{k}$. Se existe uma ação propriamente descontínua de $G$ em $X$ então $G$ é finito. De fato, dados $1 \neq g \in G$ e $x \in X$ fixado, tomamos a seqüência $S=\left(x_{n}=g^{n} x\right)_{n \geq 0} \mathrm{em} X$. Pela compacidade de $X, S$ possui um ponto de acumulação $y \in X$. Como a ação é propriamente descontínua, existe uma vizinhança aberta $U$ de $y$ tal que $g U \cap U \neq \emptyset$ implica $g=1$. Como $y$ é ponto de acumulação, existe $m$ tal que $x_{n} \in U$, para $n \geq m$, ou seja, $g^{m} x, g^{m+1} x, \ldots \in U$. Portanto $g^{m+1} x \in g U \cap U$ e então $g^{m+1}=1$. Logo $G$ é finito.

2.6 Proposição (AÇ̃̃o em dimensão PAR): Se $G$ é um grupo livre de ponto fixo que atua na esfera $S^{n}$ de dimensão par então $G=\mathbb{Z}_{2}$.

Prova: Suponha que $\varphi: G \times S^{n} \rightarrow S^{n}$ seja uma ação livre de $G$ em $S^{n}$, com $n$ par. Seja $e \neq g \in G$ e definimos $L_{g}: S^{n} \rightarrow S^{n}$ por $L_{g}(x)=\varphi(g, x)$. Desde que $L_{g}(x) \neq x$ para todo $x \in S^{n}$, então $L_{g} \sim a$, onde $a: S^{n} \rightarrow S^{n}$ é a aplicação antipodal. Portanto $\operatorname{deg}\left(L_{g}\right)=\operatorname{deg}(a)=(-1)^{n+1}$. Do mesmo modo, para outro elemento $e \neq h \in G, \operatorname{deg}\left(L_{h}\right)=(-1)^{n+1}$.

Suponha que $g h \neq e$ e assim $\operatorname{deg}\left(L_{g h}\right)=(-1)^{n+1}$. Por outro lado, $\operatorname{deg}\left(L_{g h}\right)=\operatorname{deg}\left(L_{g} L_{h}\right)=$ $\operatorname{deg}\left(L_{g}\right) \operatorname{deg}\left(L_{h}\right)=(-1)^{2(n+1)}$. Logo, $2(n+1) \equiv n+1 \bmod 2$ e assim $n$ deve ser ímpar, contradizendo a hipótese. Logo, $g h=e$ para quaisquer $g, h \in G-\{e\}$, ou seja, $G=\mathbb{Z}_{2}$. 
2.7 OBSERVAÇÃO: Se $\pi$ é equivalente a uma representação livre de ponto fixo, então $\pi$ é livre de ponto fixo. Se $\pi$ é livre de ponto fixo, então $\pi$ é 'faithful'. Se $\pi$ é livre de ponto fixo, então $\pi$ é uma soma direta de representações irredutíveis livres de ponto fixo. Se $\left\{\pi_{i}\right\}$ são livres de ponto fixo, então $\sum \pi_{i}$ é livre de ponto fixo. Se $\pi$ é uma representação sobre um subcorpo de $\mathbb{F}$, então $\pi$ é livre de ponto fixo se, e somente se, $\pi_{\mathbb{F}}$ é livre de ponto fixo.

2.8 TeOremA ([31, Teorema 5.1.2]): Dados um grupo finito $G$ e representações ortogonais irredutíveis livres de ponto fixo $\left\{\sigma_{1}, \ldots, \sigma_{r}\right\}$ de $G$, associamos a variedade Riemanniana conexa completa $^{1} S^{n-1} /\left(\sigma_{1} \oplus \cdots \oplus \sigma_{r}\right)(G), n=\sum_{i} \operatorname{deg}\left(\sigma_{i}\right)$, de dimensão $n-1$ e curvatura constante $K>0$. Toda variedade Riemanniana conexa completa de curvatura constante positiva é isométrica a uma variedade obtida deste modo e chamada de forma espacial esférica. $\left(G,\left\{\sigma_{1}, \ldots, \sigma_{r}\right\}\right)$ e $\left(G,\left\{\tau_{1}, \ldots, \tau_{s}\right\}\right)$ originam variedades isométricas se, e somente se, $r=s$ e existe uma permutação $k \rightarrow i_{k}$ de $\{1, \ldots, r\}$ e um automorfismo $\alpha$ de $G$ tal que $\tau_{k} \alpha$ é equivalente a $\sigma_{i_{k}}$.

O Teorema 2.8 acima sintetiza o trabalho de G. Vincent [29] e leva à classificação das variedades Riemannianas completas conexas de curvatura constante positiva. Tal problema ficou conhecido como Problema das Formas Espaciais Esféricas de Clifford-Klein. A abordagem utilizada foi a seguinte:

1. Encontrar condições necessárias para que um grupo abstrato finito seja livre de ponto fixo.

2. Classificar os grupos abstratos finitos que satisfazem tais condições, obtendo assim uma família $\left\{G_{\lambda}\right\}_{\lambda \in \Lambda}$ de grupos.

3. Determinar as classes de equivalência de representações complexas irredutíveis livres de ponto fixo de cada $G_{\lambda}$, aplicar o Lema 2.2 acima e o Teorema 4.7.3 de [31], obtendo as classes de equivalência de representações ortogonais irredutíveis livres de ponto fixo.

4. Determinar o grupo de automorfismos de cada $G_{\lambda}$ e decidir quando duas representações ortogonais irredutíveis livres de ponto fixo são equivalentes, módulo um automorfismo.

A Seção 5.2 de [31] apresenta alguns resultados sobre p-grupos, como por exemplo o Teorema de Sylow. Assim, as condições necessárias para que um grupo seja livre de ponto fixo são apresentadas no teorema abaixo. O caso $p=q$ é devido a Burnside [6] e o caso geral devido a Zassenhaus [33].

2.9 TEOREMA ([31, Teorema 5.3.1]): Seja $G$ um grupo finito que admite uma representação livre de ponto fixo sobre um corpo $\mathbb{F}$. Se $H$ é um subgrupo de ordem $p q$ em $G, p$ e $q$ primos, então $H$ é cíclico.

\footnotetext{
${ }^{1} \mathrm{Ou}$ seja, a conexão de Levi-Civita é completa. Ver [31, §2.1].
} 
Sejam $G$ um grupo finito e $p, q$ primos. Se todo subgrupo de ordem $p q$ em $G$ é cíclico, dizemos que $G$ satisfaz a $p q$-condição. O Teorema 2.9 diz que todo grupo livre de ponto fixo satisfaz todas as $p q$-condições. Em particular, satisfaz todas as $p^{2}$-condições. Juntamente com o Teorema 5.3.2 de [31], temos que os grupos livres de ponto fixo se dividem em duas classes. A primeira classe consiste de todos os grupos finitos nos quais todo subgrupo de Sylow é cíclico; a segunda classe consiste de todos os grupos finitos nos quais os subgrupos de Sylow ímpar são cíclicos e os 2subgrupos de Sylow são os quatérnios generalizados $\mathcal{Q}_{2^{a}}$ (ver Seção 3.1, página 31). Os grupos da primeira classe estão completamente caracterizados no teorema a seguir.

2.10 TEOREMA ([31, Teorema 5.4.1 (Burnside)]): Seja $G$ um grupo finito de ordem $N$ no qual todo subgrupo de Sylow é cíclico. Então $G$ é gerado por $\{A, B\}$ com relações

$$
A^{m}=B^{n}=1, \quad B A B^{-1}=A^{r}, \quad N=m n, \quad \operatorname{mdc}((r-1) n, m)=1, \quad r^{n} \equiv 1 \bmod m .
$$

O grupo comutador $G^{\prime}$ é gerado por $\{A\}$ e o grupo quociente $G / G^{\prime}$ é gerado pela classe $\left\{B G^{\prime}\right\}$. Seja $d$ a ordem de $r$ no grupo multiplicativo de resíduos módulo $m$ dos inteiros primos com $m$. Então $d$ divide $n$ e $G$ satisfaz todas as $p q$-condições se, e somente se, $n / d$ é divisível por todo divisor primo de $d$.

Reciprocamente, qualquer grupo dado por (2.1) tem ordem $N$ e todo subgrupo de Sylow é cíclico. Também, são equivalentes: (1) $G$ é cíclico, (2) $A=1,(3) r=1$, (4) $d=1$.

O seguinte teorema foi provado por Vincent [29, Théorème III*], utilizando cálculos diretos ao invés de representações induzidas do subgrupo $H . \phi$ denota a função de Euler ${ }^{2}$ e todas as representações são complexas de dimensão finita.

2.11 TEOREMA ([31, TEOREMA 5.5.1]): Seja $G$ um grupo de ordem $N$ que satisfaz todas as $p q$-condições e tem todo subgrupo de Sylow cíclico. Em outras palavras, $G$ tem geradores $\{A, B\}$ com relações $A^{m}=B^{n}=1, B A B^{-1}=A^{r}, N=m n=m n^{\prime} d$ e $\operatorname{mdc}((r-1) n, m)=1$, onde $d$ é a ordem ${ }^{2}$ de $r$ em $K_{m}$ e todo divisor primo de $d$ divide $n^{\prime}$. Seja $H$ o subgrupo de $G$ gerado por $\left\{A, B^{d}\right\}$. Então $H$ é subgrupo cíclico normal de ordem $m n^{\prime}$ e índice $d$ em $G$, gerado por $\left\{A B^{d}\right\}$ e suas representações irredutíveis são de grau 1 e dadas por

$$
\sigma_{k}\left(A B^{d}\right)=e^{2 \pi i k / m n^{\prime}}
$$

Seja $\pi_{k}$ a representação de $G$ induzida por $\sigma_{k}$, necessariamente de grau $d$. Então $\pi_{k}$ é equivalente a $\pi_{l}$ se, e somente se, $k \equiv l \bmod n^{\prime}$ e $k \equiv r^{u} l \bmod m$ com $0 \leq u<d$. Além disto, são equivalentes:

\footnotetext{
${ }^{2} \phi(l)$ é a ordem do grupo multiplicativo $K_{l}$ de resíduos módulo $l$ dos inteiros primos com $l$. Assim, $\phi(l)$ é o número de geradores do grupo cíclico $\mathcal{C}_{l}$ de ordem $l$.
} 
(i) $\pi$ é uma representação irredutível 'faithful' de $G$.

(ii) $\pi$ é uma representação irredutível livre de ponto fixo de $G$.

(iii) $\pi$ é equivalente a alguma $\pi_{k}, \operatorname{com} \operatorname{mdc}(k, N)=1$.

Em particular, as representações irredutíveis livres de ponto fixo de $G$ têm grau $d$ e são em quantia de $\phi(N) / d^{2}$.

O Teorema 2.11 pode ser enunciado de uma forma mais compreensiva e plausível para o estudo dos automorfismos de $G$.

2.12 TEOREMA ([31, TeOREMA 5.5.6]): Seja $G$ um grupo de ordem $N$ que satisfaz todas as $p q$-condições e tem todo subgrupo de Sylow cíclico, com geradores e relações como no Teorema

2.11. Dados $k, l \in \mathbb{Z}$ com $\operatorname{mdc}(k, m)=1=\operatorname{mdc}(l, n)$, definimos

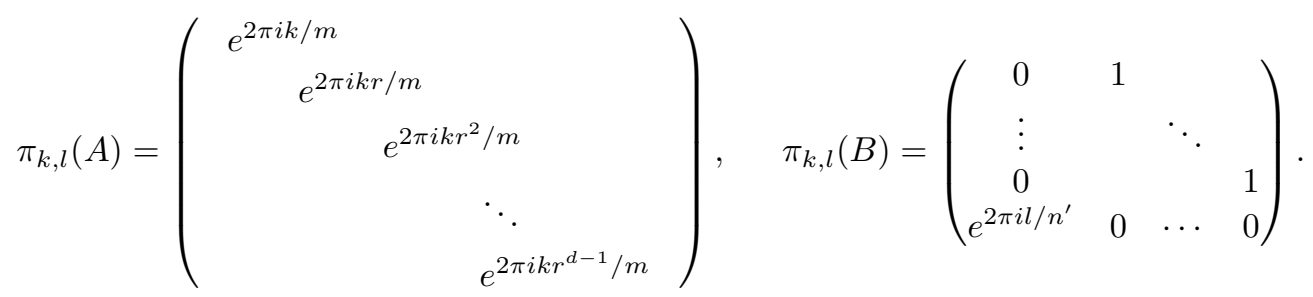

$\operatorname{Dados} s, t, u \in \mathbb{Z} \operatorname{com} \operatorname{mdc}(s, m)=1=\operatorname{mdc}(t, n)$ e $t \equiv 1 \bmod d, \operatorname{definimos}$

$$
\psi_{s, t, u}(A)=A^{s}, \quad \psi_{s, t, u}(B)=B^{t} A^{u} .
$$

Então:

1. As representações complexas irredutíveis 'faithful' de $G$ são exatamente as $\pi_{k, l}$, de grau $d$ e livres de ponto fixo.

2. Os automorfismos de $G$ são exatamente os $\psi_{s, t, u}$.

3. $\pi_{k, l} \psi_{s, t, u}$ é equivalente a $\pi_{s k, t l}$ e $\pi_{a, b}$ é equivalente a $\pi_{a^{\prime}, b^{\prime}}$ se, e somente se, $b^{\prime} \equiv b \bmod n^{\prime}$ e $a^{\prime} \equiv a r^{c} \bmod m$, para algum inteiro $c$ com $0 \leq c<d$.

4. Se $G$ é cíclico então $\pi_{k, l}$ é equivalente a sua conjugada se, e somente se, $N \leq 2$, e neste caso $\pi_{k, l}$ é equivalente a uma representação real. Se $G$ não é cíclico, então $\pi_{k, l}$ não é equivalente a uma representação real e $\pi_{k, l}$ é equivalente a sua conjugada se, e somente se, $n^{\prime}=2$.

O Teorema 2.12 nos permite descrever as representações reais livres de ponto fixo de $G$. 
2.13 TeOremA ([31, Teorema 5.5.10]): Seja $G$ um grupo de ordem $N$ que satisfaz todas as $p q$-condições e tem todo subgrupo de Sylow cíclico, ou seja, como no Teorema 2.12 acima. Suponha que $G$ não é cíclico de ordem 1 ou 2. Seja a matriz de rotação

$$
R(\theta)=\left(\begin{array}{cc}
\cos \theta & \operatorname{sen} \theta \\
-\operatorname{sen} \theta & \cos \theta
\end{array}\right)
$$

$\operatorname{Dados} k, l \in \mathbb{Z}$ com $\operatorname{mdc}(k, m)=1=\operatorname{mdc}(l, n)$, seja $\hat{\pi}_{k, l}$ a representação real de $G$ de grau $2 d$ definida (em blocos $2 \times 2$ ) por

$$
\hat{\pi}_{k, l}(A)=\left(\begin{array}{c}
R(k / m) \\
R(k r / m) \\
\ddots \\
R\left(k r^{d-1} / m\right)
\end{array}\right), \quad \hat{\pi}_{k, l}(B)=\left(\begin{array}{cccc}
0 & 1 & & \\
\vdots & & \ddots & \\
0 & & & 1 \\
R\left(l / n^{\prime}\right) & 0 & \cdots & 0
\end{array}\right) .
$$

Então, uma representação real de $G$ é livre de ponto fixo se, e somente se, é equivalente a uma soma de representações $\hat{\pi}_{k, l}$. $\hat{\pi}_{k, l}$ é equivalente a $\hat{\pi}_{k^{\prime}, l^{\prime}}$ se, e somente se, existem números $e= \pm 1 \mathrm{e}$ $c \in\{0,1, \ldots, d-1\}$ tais que $k^{\prime} \equiv e k r^{c} \bmod m$ e $l^{\prime} \equiv e l \bmod n^{\prime}$. Existem exatamente $\phi(N) / d^{2}$ ou exatamente $\phi(N) / 2 d^{2}$ representações $\hat{\pi}_{k, l}$ não equivalentes, para $n^{\prime}=2$ ou $n^{\prime} \neq 2$, respectivamente.

O lema abaixo merece destaque, pois descreve as representações para um grupo em particular que não se encaixa nos teoremas acima, pois não possui todo subgrupo de Sylow cíclico. Mais precisamente, o 2-subgrupo de Sylow do grupo dos quatérnios generalizado de ordem $2^{a}, \mathcal{Q}_{2^{a}}$, é o próprio grupo $\mathcal{Q}_{2^{a}}$, que claramente não é cíclico.

2.14 LEMA ([31, LeMA 5.6.2]): Seja $\mathcal{Q}_{2^{a}}(a \geq 3)$ o grupo dos quatérnios generalizado de ordem $2^{a}$, gerado por $\{A, B\}$ com relações

$$
A^{2^{a-1}}=1, \quad B^{2}=A^{2^{a-2}}, \quad B A B^{-1}=A^{-1} .
$$

Se $k$ é um dos $\phi\left(2^{a-2}\right)=2^{a-3}$ números $\left\{1,3,5, \ldots, 2^{a-2}-1\right\}$, definimos a representação $\alpha_{k}$ de $\mathcal{Q}_{2^{a}}$ por

$$
\alpha_{k}(A)=\left(\begin{array}{cc}
e^{2 \pi i k / 2^{a-1}} & 0 \\
0 & e^{-2 \pi i k / 2^{a-1}}
\end{array}\right), \quad \quad \alpha_{k}(B)=\left(\begin{array}{cc}
0 & -1 \\
1 & 0
\end{array}\right)
$$

Então as seguintes condições são equivalentes:

1. $\alpha$ é uma representação complexa irredutível 'faithful' de $\mathcal{Q}_{2^{a}}$.

2. $\alpha$ é uma representação complexa irredutível livre de ponto fixo de $\mathcal{Q}_{2^{a}}$.

3. $\alpha$ é equivalente a alguma $\alpha_{k}$. 
Além disto, as $\alpha_{k}$ são mutuamente não equivalentes, cada uma é equivalente a sua conjugada e nenhuma é equivalente a uma representação real.

PROVA: Claramente $\alpha_{k}$ é uma representação irredutível bem definida. Sejam $\zeta=e^{2 \pi i k / 2^{a-1}}$ e $\chi$ o caractere de $\alpha_{k}$. Como todo elemento de $\mathcal{Q}_{2^{a}}$ tem a forma $A^{t}$ ou $B A^{t}$ (ver Seção 3.1), temos que

$$
\alpha_{k}\left(A^{t}\right)=\left(\begin{array}{cc}
\zeta^{t} & 0 \\
0 & \zeta^{-t}
\end{array}\right), \quad \alpha_{k}\left(B A^{t}\right)=\left(\begin{array}{cc}
0 & -\zeta^{-t} \\
\zeta^{t} & 0
\end{array}\right)
$$

Portanto $\chi$ é uma função real e assim, pela Observação 2.1, $\alpha_{k}$ é equivalente a sua conjugada. $\alpha_{k}$ não é real pois $S O(2)$ é abeliano e $\mathcal{Q}_{2^{a}}$ não é. Os autovalores de $\alpha_{k}\left(A^{t}\right)$ e $\alpha_{k}\left(B A^{t}\right)$ são $\zeta^{ \pm t}$ e $\pm i$, respectivamente, de onde segue que $\alpha_{k}$ é livre de ponto fixo e mutuamente não equivalentes (pois possuem caracteres distintos).

Provamos então que $(3) \Rightarrow(2) \Rightarrow(1)$. Por fim, seja $\alpha$ uma representação complexa 'faithful' irredutível de $\mathcal{Q}_{2^{a}}$. Portanto, $\alpha=\sigma^{\mathcal{Q}_{2}{ }^{a}}$, com $\sigma$ representação complexa 'faithful' irredutível do grupo cíclico gerado por $\{A\}$, necessariamente da forma $\sigma\left(A^{t}\right)=\left(e^{2 \pi i k t / 2^{a-1}}\right)$, com $k$ ímpar. Assim, $\alpha$ é equivalente a $\alpha_{k}\left(\right.$ se $\left.0<k<2^{a-2}\right)$ ou $\alpha_{k-2^{a-2}}\left(\right.$ se $\left.k>2^{a-2}\right)$.

Os Teoremas 2.8 e 2.13 fornecem uma classificação completa das variedades Riemannianas conexas completas $M$ de curvatura constante positiva nas quais o grupo fundamental $G$ tem todo subgrupo de Sylow cíclico. Se $G$ é trivial então $M$ é a esfera. Se $G$ é cíclico de ordem 2 então $M$ é o espaço projetivo. Caso contrário, $M$ é isométrica a

$$
S^{2 v d-1} /\left(\hat{\pi}_{k_{1}, l_{1}} \oplus \cdots \oplus \hat{\pi}_{k_{v}, l_{v}}\right)(G)
$$

na notação do Teorema 2.13, onde $\left\{\left(k_{1}, l_{1}\right), \ldots,\left(k_{v}, l_{v}\right)\right\}$ é determinado por $M$ no sentido de que $M$ é também isométrica a uma variedade $S^{2 v d-1} /\left(\hat{\pi}_{a_{1}, b_{1}} \oplus \cdots \oplus \hat{\pi}_{a_{v}, b_{v}}\right)(G)$ se, e somente se, existe uma permutação $j \rightarrow i_{j}$ e existem $e_{j}, c_{j}, s, t \in \mathbb{Z}$ tais que $e_{j}= \pm 1,0 \leq c_{j}<d, \operatorname{mdc}(s, m)=1=$ $\operatorname{mdc}(t, n), t \equiv 1 \bmod d, k_{i_{j}} \equiv e_{j} s a_{j} r^{c_{j}} \bmod m$ e $l_{i_{j}} \equiv e_{j} t b_{j} \bmod n^{\prime}$.

\subsection{Grupos livres de ponto fixo}

Em [31, Capítulo 6] encontramos a classificação dos grupos finitos que possuem representações livres de ponto fixo, divididos em seis tipos. Os quatro primeiros tipos são para grupos solúveis e os outros dois para não solúveis. Em [31, Capítulo 7] é feita a classificação, a menos de automorfismos, das classes de equivalência de representações ortogonais de cada um dos seis tipos de grupos obtidos anteriormente. Isso soluciona o problema das formas espaciais esféricas de Clifford-Klein. 
Apresentaremos aqui, de modo resumido, apenas os resultados para os Tipos I e II definidos abaixo, que englobam o grupo cíclico e o grupo dos quatérnios generalizado $\mathcal{Q}_{2^{a}}$, como mostrado na Observação 2.15 .

\begin{tabular}{|c|l|l|l|c|}
\hline Tipo & Geradores & \multicolumn{1}{c|}{ Relações } & \multicolumn{1}{c|}{ Condições } & Ordem \\
\hline \hline \multirow{3}{*}{ I } & \multirow{2}{*}{$A, B$} & $A^{m}=B^{n}=1$, & $m \geq 1, n \geq 1$, & \\
& & $B A B^{-1}=A^{r}$ & $\operatorname{mdc}(n(r-1), m)=1$, & $m$ \\
& & $r^{n} \equiv 1 \bmod m$ & \\
\hline & & Como em I, & Como em I, & \\
& & $l^{2} \equiv r^{k-1} \equiv 1 \bmod m$, & \\
II & \multirow{2}{*}{$A, B, R$} & $R A B^{-1}=A^{l}$, & $n=2^{u} v, u \geq 2$, & $2 m n$ \\
& & $R B R^{-1}=B^{k}$ & $k \equiv-1 \bmod 2^{u}$, & \\
& & & $k^{2} \equiv 1 \bmod m$ & \\
\hline
\end{tabular}

Tabela 2.1: Dois tipos de grupos livres de ponto fixo. Para os outros quatro tipos, ver a tabela completa em [31, pág. 179].

2.15 OBSERVAÇÃ O: O grupo cíclico $\mathcal{C}_{k}$ de ordem $k$ é dado por I, gerado por $\{B\}$, com $n=k$ e $m=r=1$. O grupo dos quatérnios generalizado $\mathcal{Q}_{2^{a}}(a \geq 3)$ é dado por II, gerado por $\{B, R\}$, com $m=1, n=2^{a-1}, u=2, v=2^{a-3}, k=-1, r, l \in \mathbb{Z}$.

Seja $G$ um grupo finito que admite uma representação livre de ponto fixo. Seja $\mathfrak{F}_{\mathbb{C}}(G)$ o conjunto de todas as classes de equivalência de representações complexas irredutíveis livres de ponto fixo de $G$. Descreveremos $\mathfrak{F}_{\mathbb{C}}(G)$, estabelecendo uma notação para seus elementos. Em [31, Teorema 7.2.18] há uma descrição completa de $\mathfrak{F}_{\mathbb{C}}(G)$, para os outros quatro tipos de grupos.

Tipo I. $G$ é gerado por $\{A, B\} \operatorname{com} A^{m}=B^{n}=1, B A B^{-1}=A^{r}$, onde $m \geq 1, n \geq 1$, $r^{n} \equiv 1 \bmod m, \operatorname{mdc}(n(r-1), m)=1$ e todo divisor primo da ordem $d$ de $r$ em $K_{m}$ divide $n^{\prime}=n / d$. Então $\mathfrak{F}_{\mathbb{C}}\left(\left\langle A, B^{d}\right\rangle\right)$ consiste de todas

$$
\sigma_{k} \otimes \sigma_{l}: A^{u} B^{v d} \mapsto e^{2 \pi i k u / m} e^{2 \pi i l v / n^{\prime}}, \quad \operatorname{mdc}(k, m)=1=\operatorname{mdc}(l, n) .
$$

Assim, $\mathfrak{F}_{\mathbb{C}}(G)$ consiste das $\phi(m n) / d^{2}$ representações de grau $d$ dadas por

$$
\pi_{k, l}=\left(\sigma_{k} \otimes \sigma_{l}\right)^{G}, \quad \sigma_{k} \otimes \sigma_{l} \in \mathfrak{F}_{\mathbb{C}}\left(\left\langle A, B^{d}\right\rangle\right) .
$$

$\pi_{k, l}=\pi_{a, b}$ se, e somente se, $k \equiv a r^{u} \bmod m$ e $l d \equiv b d \bmod n, \operatorname{com} 0 \leq u<d$. Estes fatos estão contidos no Teorema 2.11 .

2.16 OBSERVAÇÃO: Em particular, para o grupo cíclico $\mathcal{C}_{n}$ de ordem $n$, para cada $l$ primo com $n$ temos uma representação complexa irredutível livre de ponto fixo $\pi_{l}$, de grau 1 . Como $\pi_{a}=\pi_{b}$ se, e somente se, $a \equiv b \bmod n$, basta considerarmos $l<n$ e assim existem $\phi(n)$ tais representações não equivalentes. 
Tipo II. $G$ é gerado por $\{A, B, R\}$ onde $\langle A, B\rangle$ é do tipo I, $R$ normaliza ambos $\{A\}$ e $\{B\}$ e $n_{1} \equiv 0 \bmod 4$. Então $\mathfrak{F}_{\mathbb{C}}(G)$ consiste das $\phi(m n) / 2 d^{2}=\phi(2 m n) /(2 d)^{2}$ representações de grau $2 d$ dadas por

$$
\alpha_{k, l}=\pi_{k, l}^{G}, \quad \pi_{k, l} \in \mathfrak{F}_{\mathbb{C}}(\langle A, B\rangle)
$$

onde $\alpha_{k, l}=\alpha_{a, b}$ se, e somente se, $k \equiv \varepsilon a r^{u} \bmod m_{1}, k \equiv a r^{u} \bmod m_{2}, l d \equiv \varepsilon b d \bmod n_{1}$ e $l d \equiv b d \bmod n_{2}, \operatorname{com} \varepsilon= \pm 1$ e $0 \leq u<d$. Para as definições de $m_{i}$ e $n_{i}$, ver [31, Seção 7.2].

2.17 ОBSERVAÇÃO: Em particular, para o grupo $\mathcal{Q}_{2^{a}}$, como observado em $2.15,\langle B\rangle=\mathcal{C}_{2^{a-1}}$ e $\operatorname{assim} n=n_{1}=2^{a-1}\left(n_{1} \equiv 0 \bmod 4\right.$ pois $\left.a \geq 3\right)$. Para cada uma das $\phi\left(2^{a-1}\right)$ representações complexas $\pi_{l} \in \mathfrak{F}_{\mathbb{C}}\left(\mathcal{C}_{2^{a-1}}\right)$, temos a representação induzida $\alpha_{l}=\pi_{l}^{\mathcal{Q}_{2} a}$ de grau $2(d=1)$. Porém, se $l>2^{a-2}$ então $l \equiv-b \bmod 2^{a-1}, \operatorname{com} b<2^{a-2}$. Logo, podemos dividir pela metade a quantia de representações, ou seja, existem $\phi\left(2^{a-1}\right) / 2=2^{a-3}$ representações complexas $\alpha_{l}$ não equivalentes, como afirmado acima.

Ação dos automorfismos nas representações: Seja $G$ um grupo finito que admite uma representação complexa livre de ponto fixo. Dado o conjunto $\mathfrak{F}_{\mathbb{C}}(G)$ de todas as classes de equivalência de representações complexas irredutíveis livres de ponto fixo, os automorfismos de $G$ atuam em $\mathfrak{F}_{\mathbb{C}}(G)$ por composição, $\psi: \pi \mapsto \pi \psi$. Definimos $\mathfrak{A}_{\mathbb{C}}(G)$ como sendo o grupo das transformações de $\mathfrak{F}_{\mathbb{C}}(G)$ induzidas por automorfismos de $G$.

A tabela abaixo resume a situação para os grupos dos Tipos I e II. Em [31, Teorema 7.3.21] temos a descrição completa para os outros tipos.

\begin{tabular}{|c|c|c|l|}
\hline Tipo & Elementos de $\mathfrak{A}_{\mathbb{C}}(G)$ & $\operatorname{Ação~em~} \mathfrak{F}_{\mathbb{C}}(G)$ & Condições \\
\hline \hline I & & $\pi_{k, l} \rightarrow \pi_{a k, b l}$ & $\operatorname{mdc}(a, m)=1$ \\
\cline { 1 - 2 } II & $A_{a, b}$ & $\operatorname{mdc}(b, n)=1$ \\
& & $\alpha_{k, l} \rightarrow \alpha_{a k, b l}$ & $b \equiv 1 \bmod d$ \\
\hline
\end{tabular}

Tabela 2.2: Ação dos automorfismos de $G$ em $\mathfrak{F}_{\mathbb{C}}(G)$. Para os outros quatro tipos, ver a tabela completa em [31, pág. 217].

\subsection{Classificação das formas espaciais esféricas}

Os Teoremas 5.1.2, 7.2.18 e 7.3.21 de [31] classificam as variedades Riemannianas conexas completas $M$ de curvatura constante positiva, a menos de isometria global. O Teorema 5.1.2 é o Teorema 2.8 na página 22 deste trabalho e os Teoremas 7.2.18 e 7.3.21 englobam os resultados (particularizados) das Seções 2.2 e 2.2 acima. 
Seja $G$ o grupo fundamental de $M$. Então $M$ é obtida como segue: se $G$ tem ordem 1 ou 2 , então $M$ é isométrica à esfera ou ao espaço projetivo, respectivamente, de dimensões apropriadas.

Se $G$ é do Tipo I com ordem maior que dois, então $M$ é isométrica a alguma

$$
S^{2 s d-1} /\left(\hat{\pi}_{k_{1}, l_{1}} \oplus \cdots \oplus \hat{\pi}_{k_{s}, l_{s}}\right)(G)
$$

onde $^{3}\left(\hat{\pi}_{k, l}\right)_{\mathbb{C}}=\pi_{k, l} \oplus \bar{\pi}_{k, l}$. O conjunto $\left\{\left(k_{i}, l_{i}\right)\right\}$ é determinado a menos de uma transformação $\left(k_{i}, l_{i}\right) \rightarrow\left(x_{i}, y_{i}\right)$ para a qual existe uma permutação $i \rightarrow i^{\prime}$ de $\{1, \ldots, s\}$ e existem $e_{i}, u_{i}, a, b \in \mathbb{Z}$ tais que

$$
\begin{gathered}
e_{i}= \pm 1, \quad 0 \leq u_{i}<d, \quad \operatorname{mdc}(a, m)=\operatorname{mdc}(b, n)=1, \quad b \equiv 1 \bmod d \\
x_{i^{\prime}} \equiv e_{i} a k_{i} r^{u_{i}} \bmod m, \quad y_{i^{\prime}} d \equiv e_{i} b l_{i} d \bmod n .
\end{gathered}
$$

Se $G$ é do Tipo II, então $M$ é isométrica a alguma

$$
S^{4 s d-1} /\left(\hat{\alpha}_{k_{1}, l_{1}} \oplus \cdots \oplus \hat{\alpha}_{k_{s}, l_{s}}\right)(G)
$$

$\operatorname{onde}^{3}\left(\hat{\alpha}_{k, l}\right)_{\mathbb{C}}=\alpha_{k, l} \oplus \bar{\alpha}_{k, l}$. O conjunto $\left\{\left(k_{i}, l_{i}\right)\right\}$ é determinado a menos de uma transformação $\left(k_{i}, l_{i}\right) \rightarrow\left(x_{i}, y_{i}\right)$ para a qual existe uma permutação $i \rightarrow i^{\prime}$ de $\{1, \ldots, s\}$ e existem $e_{i}, \varepsilon_{i}, u_{i}, a, b \in \mathbb{Z}$ tais que

$$
\begin{gathered}
e_{i}= \pm 1, \quad \varepsilon_{i}= \pm 1, \quad 0 \leq u_{i}<d, \quad \operatorname{mdc}(a, m)=\operatorname{mdc}(b, n)=1, \quad b \equiv 1 \bmod d \\
x_{i^{\prime}} \equiv \varepsilon_{i} e_{i} a k_{i} r^{u_{i}} \bmod m_{1}, \quad x_{i^{\prime}} \equiv e_{i} a k_{i} r^{u_{i}} \bmod m_{2} \\
y_{i^{\prime}} d \equiv \varepsilon_{i} e_{i} b l_{i} d \bmod n_{1}, \quad y_{i^{\prime}} d \equiv e_{i} b l_{i} d \bmod n_{2} .
\end{gathered}
$$

Os demais tipos estão descritos em [31, Seção 7.4].

2.18 OBSERVAÇÃo (Espaços lenticulares): Considerando o grupo cíclico $\mathcal{C}_{n}$, de ordem $n$, gerado por $\{B\}$, como em 2.15, temos que as representações reais são de grau 2 e dadas por (2.4), ou seja, $\hat{\pi}_{l}(B)=R(l / n)$, para cada $l$ primo com $n(l<n)$. A ordem dos índices $l_{i}$ não importa e se $l_{i^{\prime}} \equiv \pm b l_{i} \bmod n, \operatorname{com} b \equiv 1 \bmod d$, obtemos variedades isométricas. Na notação do Apêndice A, temos

$$
S^{2 s-1} /\left(\hat{\pi}_{l_{1}} \oplus \cdots \oplus \hat{\pi}_{l_{s}}\right)\left(\mathcal{C}_{n}\right)=L\left(n ; l_{1}, \ldots, l_{s}\right)
$$

2.19 OBSERVAÇÃO: Em [16, §4.2.5] são apresentadas formas espaciais esféricas com o mesmo espectro, com o mesmo grupo fundamental não abeliano, mas que não são isométricas. Porém, em [16, Teorema 4.2.15], são exibidos espaços lenticulares (grupo fundamental abeliano) que possuem o mesmo espectro mas não são isométricos.

\footnotetext{
${ }^{3}$ Lembramos que uma representação real $\omega$ pode ser vista como uma representação complexa $\omega_{\mathrm{C}}$ e que $\eta \oplus \bar{\eta}$ é da forma $\omega_{\mathbb{C}}$ para toda representação complexa $\eta$.
} 



\section{FORMAS ESPACIAIS ESFÉRICAS QUATERNIÔNICAS}

Neste capítulo, apresentamos o grupo dos quatérnios generalizado $\mathcal{Q}_{4 t}$, também conhecido por grupo quaterniônico. Estudamos a ação deste grupo nas esferas de dimensão ímpar, construímos um complexo de cadeias coerente com tal ação a fim de lidarmos com o espaço quociente, o qual chamaremos de forma espacial esférica quaterniônica. Este capítulo fornecerá o material necessário para o cálculo da $\mathrm{R}$ torção destes espaços.

\subsection{Grupo dos quatérnios generalizado $\mathcal{Q}_{4 t}$}

Seja $\mathcal{Q}_{4 t}=\left\langle x, y: x^{t}=y^{2}, y x y^{-1}=x^{-1}\right\rangle$ o grupo dos quatérnios generalizado, de ordem $4 t$. Como de costume, escrevemos $x^{0}=1$, o elemento neutro do grupo. Podemos então obter algumas relações úteis:

1. $x^{-k}=\left(y x y^{-1}\right) \cdots\left(y x y^{-1}\right)=y x^{k} y^{-1}$.

2. $y x^{k}=x^{-k} y$.

3. $x^{k} y x^{k}=y$.

4. $x^{-t}=y x^{t} y^{-1}=y y^{2} y^{-1}=y^{2}=x^{t}$ e assim $1=x^{2 t}=y^{4}$.

5. $y x y=y x\left(y^{-1} y\right) y=x^{-1} y^{2}=x^{-1} x^{t}=x^{t-1}$.

6. $x y x=x y x\left(y^{-1} y\right)=x\left(x^{-1}\right) y=y$.

7. $y^{-1} x y=y^{-2}\left(y x y^{-1}\right) y^{2}=y^{-2} x^{-1} y^{2}=x^{-t} x^{-1} x^{t}=x^{-1}$.

8. $x^{-k} y x^{k}=\left(y x^{k} y^{-1}\right)\left(y x^{k}\right)=y x^{2 k}$.

Resumindo:

$$
\begin{aligned}
& x^{t}=y^{2} \quad y x^{k} y^{-1}=x^{-k} \\
& y x y=x^{t-1} \\
& x y x=y \\
& x^{k} y x^{k}=y \\
& 1=x^{2 t}=y^{4} \\
& y^{-1} x y=x^{-1} \\
& x^{-k} y x^{k}=y x^{2 k} \\
& y x^{k}=x^{-k} y
\end{aligned}
$$

Então, podemos listar os elementos, ou seja,

$$
\mathcal{Q}_{4 t}=\left\{1, x, x^{2}, \ldots, x^{2 t-1}, y, x y, \ldots, x^{2 t-1} y\right\}
$$


pois

$$
x^{k} y^{2}=x^{k} x^{t}=x^{k+t}=x^{j}, \quad x^{k} y^{3}=x^{k} y^{2} y=x^{j} y,
$$

para $0 \leq j \leq 2 t-1$. Logo, $\left|\mathcal{Q}_{4 t}\right|=4 t$

3.1 OBSERVAÇÃO: Podemos classificar o grupo dos quatérnios generalizado de acordo com $t$, ou seja, para $a \geq 3$ e $t=2^{a-2}$ temos $\mathcal{Q}_{2^{a}}$; para $q$ ímpar, $i \geq 1$ e $t=2^{i} q$ temos $\mathcal{Q}_{2^{i+2} q}$; para $t$ ímpar temos $\mathcal{Q}_{4 t}$.

Em [31], o termo 'quaterniônico generalizado' é associado apenas ao grupo $\mathcal{Q}_{2^{a}}$. Porém, os três tipos estão descritos na Tabela 2.1 (ou [31, pag. 179]). A correspondência entre os geradores $A, B, R$ (de [31]) e os geradores $x, y$ (deste texto) segue.

Tipo $\mathcal{Q}_{2^{a}}=\mathcal{Q}_{4 t}, a \geq 3, t=2^{a-2}$ : Tipo II na Tabela $2.1 \mathrm{com}$

$$
\begin{aligned}
& A=1, \quad B=x, \quad R=y, \\
& m=1, \quad n=2^{a-1}, \quad u=2, \quad v=2^{a-3}, \quad k=-1, \quad(r, l \in \mathbb{Z})
\end{aligned}
$$

e assim

$$
\begin{gathered}
B^{n}=x^{2^{a-1}}=x^{2 t}=1, \quad R^{2}=y^{2}=x^{t}=B^{2^{a-2}}=B^{n / 2}, \\
R B R^{-1}=y x y^{-1}=x^{-1}=B^{-1} .
\end{gathered}
$$

Tipo $\mathcal{Q}_{4 t}, t=2^{i} q, q$ ímpar, $i \geq 1$ : Tipo II na Tabela 2.1 com

$$
\begin{aligned}
& A=x^{2^{i+1}}, \quad B=x^{q}, \quad \quad R=y, \quad m=q, \quad n=2^{i+1}, \\
& r=1, \quad l=k=-1, \quad u=2, \quad v=2^{i-1},
\end{aligned}
$$

e assim

$$
\begin{aligned}
A^{m}= & x^{2^{i+1} q}=x^{2 t}=B^{n}, \quad R^{2}=y^{2}=x^{2^{i} q}=B^{2^{i}}=B^{n / 2}, \\
& B A B^{-1}=x^{q} x^{2^{i+1}} x^{-q}=x^{2^{i+1}}=A^{r}, \\
& R A R^{-1}=y x^{2^{i+1}} y^{-1}=\left(x^{-1}\right)^{2^{i+1}}=A^{-1}=A^{k}, \\
& R B R^{-1}=y x^{q} y^{-1}=\left(x^{-1}\right)^{q}=B^{-1}=B^{l} .
\end{aligned}
$$

Tipo $\mathcal{Q}_{4 t}, t$ ímpar: Tipo I na Tabela $2.1 \mathrm{com}$

$$
A=x^{2}, \quad B=y, \quad m=t, \quad n=4, \quad r=-1,
$$

e assim

$$
\begin{gathered}
A^{m}=x^{2 t}=y^{4}=B^{n}=1 \\
B A B^{-1}=y x^{2} y^{-1}=\left(y x y^{-1}\right)\left(y x y^{-1}\right)=x^{-2}=A^{r} .
\end{gathered}
$$




\subsection{Representações de $\mathcal{Q}_{4 t}$}

Seja $H$ subgrupo de $G$, de índice $n$. Se $\sigma$ é uma representação de $H$ em $U$, então existe uma representação induzida $\sigma^{G} \operatorname{de} G$ em $V=U \oplus \cdots \oplus U$ ( $n$ vezes) dada por $\sigma^{G}(g)=\left(\sigma\left(b_{i}^{-1} g b_{j}\right)_{i j}\right)$, ou seja, a entrada $(i, j)$ da matriz de $\sigma^{G}(g)$ é $\sigma\left(b_{i}^{-1} g b_{j}\right)$, onde $G=\bigcup_{i=1}^{n} b_{i} H, b_{1} \in H$, e $\sigma(c)=0$ para $c \notin H$.

Assim sendo, escrevemos $\mathcal{Q}_{4 t}=\mathcal{C}_{2 t} \cup y \mathcal{C}_{2 t}$, onde $\mathcal{C}_{2 t}=\left\langle x: x^{2 t}=1\right\rangle$ é o subgrupo cíclico de índice 2 e ordem $2 t$. Seja $\left\{b_{1}=1, b_{2}=y\right\}$ conjunto de representantes para as classes laterais. Note que $y \mathcal{C}_{2 t}=y x \mathcal{C}_{2 t}$ e outras escolhas para $b_{2}$ dão origem à representações equivalentes. Assim, dada uma representação complexa $\pi_{k}(\operatorname{mdc}(k, 2 t)=1)$ para $\mathcal{C}_{2 t}$, como na Observação 2.16 , temos a representação complexa induzida $\pi_{k}^{\mathcal{Q}_{4 t}}$, cuja matriz de $\pi_{k}^{\mathcal{Q}_{4 t}}(g)$ é definida em blocos por

$$
\pi_{k}^{\mathcal{Q}_{4 t}}(g)=\left(\begin{array}{cc}
\pi_{k}\left(b_{1}^{-1} g b_{1}\right) & \pi_{k}\left(b_{1}^{-1} g b_{2}\right) \\
\pi_{k}\left(b_{2}^{-1} g b_{1}\right) & \pi_{k}\left(b_{2}^{-1} g b_{2}\right)
\end{array}\right) .
$$

De modo mais específico, temos nos geradores $x, y$ :

$$
\begin{aligned}
& \pi_{k}^{\mathcal{Q}_{4 t}}(x)=\left(\begin{array}{cc}
\pi_{k}(x) & \pi_{k}(x y) \\
\pi_{k}\left(y^{-1} x\right) & \pi_{k}\left(x^{-1}\right)
\end{array}\right)=\left(\begin{array}{cc}
e^{2 \pi i k / 2 t} & 0 \\
0 & e^{-2 \pi i k / 2 t}
\end{array}\right)=\left(\begin{array}{cc}
e^{\pi i k / t} & 0 \\
0 & e^{-\pi i k / t}
\end{array}\right), \\
& \pi_{k}^{\mathcal{Q}_{4 t}}(y)=\left(\begin{array}{cc}
\pi_{k}(y) & \pi_{k}\left(y^{2}\right) \\
\pi_{k}(1) & \pi_{k}(y)
\end{array}\right)=\left(\begin{array}{cc}
0 & e^{\pi i} \\
e^{2 \pi i} & 0
\end{array}\right)=\left(\begin{array}{cc}
0 & -1 \\
1 & 0
\end{array}\right),
\end{aligned}
$$

coincidindo com (2.6) do Lema 2.14 (para $A=x$ e $B=y$ ). Note que

$$
\begin{aligned}
& \pi_{k}^{\mathcal{O}_{4 t}}(y x)=\left(\begin{array}{cc}
\pi_{k}(y x) & \pi_{k}\left(x^{t-1}\right) \\
\pi_{k}(x) & \pi_{k}(x y)
\end{array}\right)= \\
&=\left(\begin{array}{cc}
0 & e^{2 \pi i k(t-1) / 2 t} \\
e^{2 \pi i k / 2 t} & 0
\end{array}\right)=\left(\begin{array}{cc}
0 & -e^{-2 \pi i k / 2 t} \\
e^{2 \pi i k / 2 t} & 0
\end{array}\right)= \\
&\left(\begin{array}{cc}
0 & -1 \\
1 & 0
\end{array}\right)\left(\begin{array}{cc}
e^{2 \pi i k / 2 t} & 0 \\
0 & e^{-2 \pi i k / 2 t}
\end{array}\right)=\pi_{k}^{\mathcal{Q}_{4 t}}(y) \pi_{k}^{\mathcal{Q}_{4 t}}(x),
\end{aligned}
$$

já que $\pi\left(g_{1} g_{2}\right)=\pi\left(g_{1}\right) \pi\left(g_{2}\right)$. 


\subsection{Região fundamental para a ação de $\mathcal{Q}_{4 t}$ nas esferas}

\subsubsection{Notações}

A partir daqui, $\theta=\pi / t$ e $\zeta=e^{i \theta}$ é uma raíz primitiva $2 t$-ésima da unidade. Também, $q_{1}, q_{2}, \ldots, q_{n}$ são inteiros (não necessariamente distintos) $\operatorname{com} \operatorname{mdc}\left(q_{j}, 2 t\right)=1$ e $r_{1}, r_{2}, \ldots, r_{n}$ são os inversos multiplicativos módulo $2 t$, isto é, $q_{j} r_{j} \equiv 1 \bmod 2 t$. Como de costume, identificamos $\mathbb{C}^{n}$ com $\mathbb{R}^{2 n}$ de modo que cada cópia de $\mathbb{C}$ seja gerada pelos vetores canônicos $e_{i}, e_{i+1} \in \mathbb{R}^{2 n}$. Deste modo, o plano gerado por $\left\{e_{p}, e_{q}\right\}$ será chamado de plano-pq.

A região fundamental para a ação de $\mathcal{Q}_{\mu}$ em $S^{\nu}$ será denotada por $\mathcal{F}_{\mu, \nu}$.

3.2 OBSERVAÇÃO: Suponhamos que plano-pq e plano-rs interceptam-se apenas na origem. Sejam $C_{1}, C_{2}$ subconjuntos das circunferências de raio 1 centradas na origem contidas no $p l a n o-p q$ e no plano-rs, respectivamente. Dados dois pontos $A \in C_{1}$ e $B \in C_{2}$, existem $\alpha, \beta \in[0,2 \pi]$ tais que $A=(\ldots, 0, \cos \alpha, \operatorname{sen} \alpha, 0, \ldots)$ e $B=(\ldots, 0, \cos \beta, \operatorname{sen} \beta, 0, \ldots)$, ou seja, as únicas entradas não nulas de $A$ (resp. $B$ ) são $p, q$ (resp. $r, s$ ). Assim, existe um plano em $\mathbb{C}^{n}$ passando pela origem e por $A, B$, interceptando $S^{2 n-1}$ em uma circunferência de raio 1 . Como os vetores $\vec{A}, \vec{B}$ são ortogonais, então o arco menor que liga $A$ a $B$ tem comprimento $\pi / 2$. Tais arcos serão usados para a descrição da região fundamental.

3.3 DEFINIÇÃO ('CURved JoIN'): Na situação descrita em 3.2 acima, o conjunto de todos os arcos de comprimento $\pi / 2$ que ligam pontos de $C_{1}$ com pontos de $C_{2}$ será chamado de 'curved join' e denotado por $C_{1} \tilde{*} C_{2}$. Difere do 'join' convencional pois este último é construído através de segmentos e não arcos (em uma situação mais geral).

Sejam $\Sigma_{j}$ a 1-esfera unitária $S^{1}$ na $j$-ésima cópia de $\mathbb{C}$ em $\mathbb{C}^{2 n}$ e $\Sigma^{j}=\Sigma_{1} \tilde{*} \ldots \tilde{*} \Sigma_{j}$, com $\Sigma^{0}=\emptyset$, de modo que a esfera unitária $S^{4 n-1}$ nada mais é que $\Sigma^{2 n}=\Sigma_{1} \tilde{*} \cdots \tilde{*} \Sigma_{2 n}$. Os pontos $v_{j}=e^{\pi i / 2} \in \Sigma_{2 j}$ e os $\operatorname{arcos} I_{j}=\left[v_{j}, x^{r_{j}} v_{j}\right] \subset \Sigma_{2 j}$ e $J_{j}=\left[v_{j}, y v_{j}\right]$, ligando $v_{j}$ a $x^{r_{j}} v_{j}$ e $v_{j}$ a $y v_{j}$, respectivamente, serão relevantes para a construção da região fundamental $\mathcal{F}_{4 t, 4 n-1}$.

\subsubsection{Ação de $\mathcal{Q}_{8}$ em $S^{3}$}

Descreveremos aqui o processo de construção da região fundamental $\mathcal{F}_{8,3}$. Optamos por começar com o caso particular $t=2, n=1$, pois assim a notação mais geral introduzida acima torna-se mais clara e auto-explicativa. Lembramos que a esfera unitária $S^{3} \subset \mathbb{R}^{4}$ possui como "equador" a esfera $S^{2}$ e que os "pólos norte/sul" são os pontos $\pm e_{4}$.

Seja $\alpha: \mathcal{Q}_{8} \rightarrow U(2, \mathbb{C})$ dada por

$$
\alpha(x)=\left(\begin{array}{cc}
i & 0 \\
0 & -i
\end{array}\right) \quad \text { e } \quad \alpha(y)=\left(\begin{array}{cc}
0 & -1 \\
1 & 0
\end{array}\right)
$$


como em (3.1) e (3.2), para $k=1$. Portanto, podemos considerar $\alpha(x), \alpha(y): \mathbb{C}^{2} \rightarrow \mathbb{C}^{2}$. Uma base ordenada sobre $\mathbb{R}$ para $\mathbb{C}^{2}$ é $\{(1,0),(i, 0),(0,1),(0, i)\}$ e nesta base escrevemos

$$
\begin{array}{ll}
\alpha(x)(1,0)=(i, 0) \equiv(0,1,0,0) \in \mathbb{R}^{4} & \alpha(y)(1,0)=(0,1) \equiv(0,0,1,0) \in \mathbb{R}^{4} \\
\alpha(x)(i, 0)=(-1,0) \equiv(-1,0,0,0) \in \mathbb{R}^{4} & \alpha(y)(i, 0)=(0, i) \equiv(0,0,0,1) \in \mathbb{R}^{4} \\
\alpha(x)(0,1)=(0,-i) \equiv(0,0,0,-1) \in \mathbb{R}^{4} & \alpha(y)(0,1)=(-1,0) \equiv(-1,0,0,0) \in \mathbb{R}^{4} \\
\alpha(x)(0, i)=(0,1) \equiv(0,0,1,0) \in \mathbb{R}^{4} & \alpha(y)(0, i)=(-i, 0) \equiv(0,-1,0,0) \in \mathbb{R}^{4}
\end{array}
$$

ou seja,

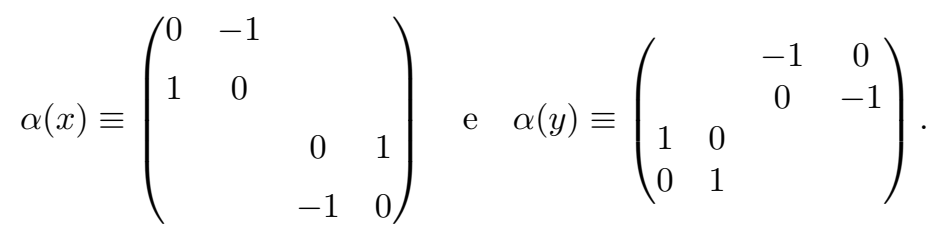

Calculamos então a ação de $x, y, x y$ em cada $e_{i}$, obtendo

$$
\begin{aligned}
& x \cdot e_{1}=e_{2} \quad x \cdot e_{2}=-e_{1} \quad x \cdot e_{3}=-e_{4} \quad x \cdot e_{4}=e_{3} \\
& y \cdot e_{1}=e_{3} \quad y \cdot e_{2}=e_{4} \quad y \cdot e_{3}=-e_{1} \quad y \cdot e_{4}=-e_{2} \\
& x y \cdot e_{1}=-e_{4} \quad x y \cdot e_{2}=e_{3} \quad x y \cdot e_{3}=-e_{2} \quad x y \cdot e_{4}=e_{1}
\end{aligned}
$$

Por simplicidade, escreveremos $g e_{i}$ para a ação de $g$ em $e_{i}$. A Figura 3.1 mostra tais ações, lembrando que os segmentos que possuem $\pm e_{4}$ como um de seus vértices são, na verdade, arcos de comprimento $\pi / 2$.
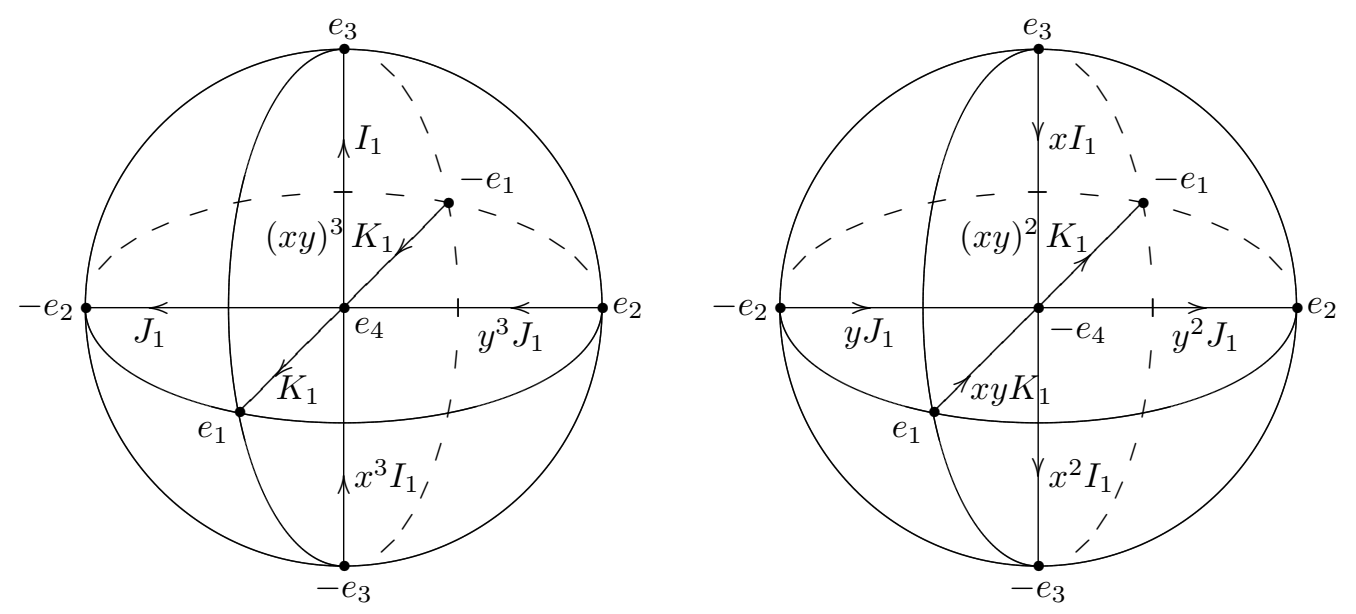

Figura 3.1: Representação tridimensional para a esfera $S^{3}$. 
3.4 OBSERVAÇÃ̃: Notemos que a ação de $x$ corresponde a uma rotação de $\theta$ no sentido horário (resp. anti-horário) no plano-34 (resp. plano-12) e que a ação de y (também de $x y$ ) aplica o plano-12 no plano-34 e o plano-34 no plano-12 seguido de uma rotação de $\pi$.

Deste modo, $\mathcal{F}_{8,3}$ é mostrado na Figura 3.2 e terá como 0-células os pontos

$$
e_{4}, \quad x e_{4}, \quad y e_{4}, \quad x y e_{4}, \quad x^{2} e_{4}=y^{2} e_{4}=(x y)^{2} e_{4}=-e_{4},
$$

como 1-células os arcos

$$
\begin{aligned}
& I_{1}=\left[e_{4}, x e_{4}\right], \quad J_{1}=\left[e_{4}, y e_{4}\right], \quad K_{1}=\left[e_{4}, x y e_{4}\right], \\
& x I_{1}=\left[x e_{4}, x^{2} e_{4}\right], \quad x J_{1}=\left[x e_{4}, x y e_{4}\right], \quad x y K_{1}=\left[x y e_{4},(x y)^{2} e_{4}\right] \text {, } \\
& x y I_{1}=\left[x y e_{4}, y e_{4}\right], \quad y J_{1}=\left[y e_{4}, y^{2} e_{4}\right],
\end{aligned}
$$

como 2-células os "simplexos" $\sigma_{1}, \sigma_{2}, \sigma_{3}$ abaixo (observo que apesar de usarmos a notação simplicial para descrevermos $\sigma_{i}$, são na verdade células)

$$
\begin{aligned}
\sigma_{1} & =\left[e_{4}, y e_{4}, x e_{4}\right]+\left[x e_{4}, y e_{4}, x^{2} e_{4}\right], \\
\sigma_{2} & =\left[e_{4}, x e_{4}, x y e_{4}\right]+\left[x y e_{4}, y e_{4}, e_{4}\right], \\
\sigma_{3} & =\left[x e_{4}, x^{2} e_{4}, x y e_{4}\right]+\left[x y e_{4}, x^{2} e_{4}, y e_{4}\right]
\end{aligned}
$$

e uma única 3-célula $\gamma$, que pode ser descrita como $\gamma=(1+x) I_{1} \tilde{*} x y I_{1}$.
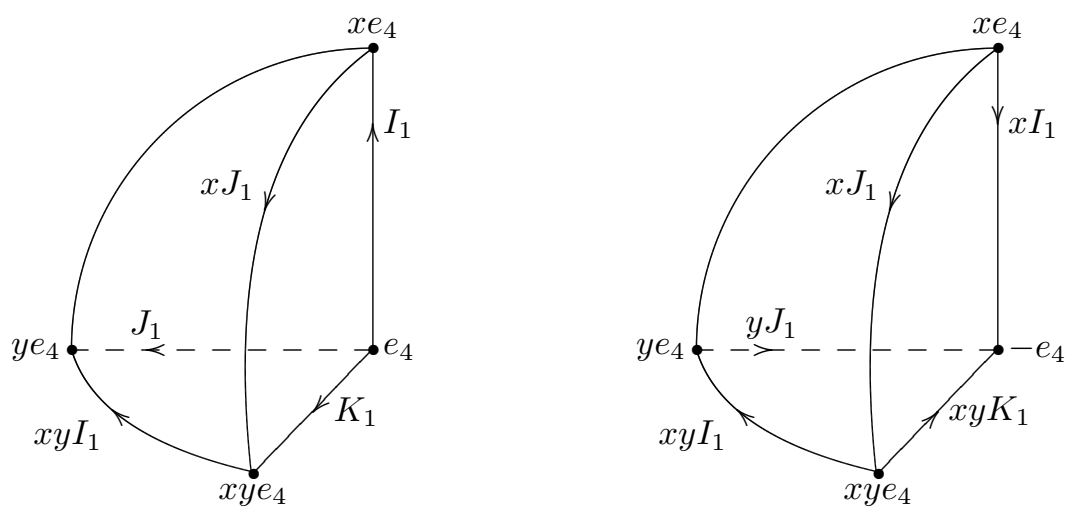

Figura 3.2: Região fundamental $\mathcal{F}_{8,3}$.

Podemos escolher dentre as células acima um conjunto de representantes (células fundamentais) em cada dimensão, obtendo o complexo de cadeias (3.3) sobre o anel de grupos $\mathbb{Z}\left[\mathcal{Q}_{8}\right]$, possuindo apenas uma 0-célula, duas 1-células, duas 2-células e uma 3-célula.

$$
0 \longrightarrow \mathbb{Z}\left[\mathcal{Q}_{8}\right]\langle\gamma\rangle \stackrel{\partial_{3}}{\longrightarrow} \mathbb{Z}\left[\mathcal{Q}_{8}\right]\left\langle\sigma_{1}, \sigma_{2}\right\rangle \stackrel{\partial_{2}}{\longrightarrow} \mathbb{Z}\left[\mathcal{Q}_{8}\right]\left\langle I_{1}, J_{1}\right\rangle \stackrel{\partial_{1}}{\longrightarrow} \mathbb{Z}\left[\mathcal{Q}_{8}\right]\left\langle e_{4}\right\rangle \longrightarrow 0
$$


Denotando uma $k$-célula fundamental por $\tilde{c}^{k}$, então

$$
\begin{array}{lll}
\tilde{c}^{0}=e_{4}, & \tilde{c}_{1}^{2}=\sigma_{1}, & \tilde{c}^{3}=\gamma, \\
& \tilde{c}_{2}^{1}=J_{1} & \tilde{c}_{2}^{2}=\sigma_{2},
\end{array}
$$

pois $\sigma_{3}=-x \sigma_{1}-x y \sigma_{2}$. Os bordos são

$$
\begin{array}{ll}
\partial_{1}\left(\tilde{c}_{1}^{1}\right)=(1-x) \tilde{c}^{0}, & \partial_{2}\left(\tilde{c}_{1}^{2}\right)=-(1+x) \tilde{c}_{1}^{1}+(1+y) \tilde{c}_{2}^{1}, \\
\partial_{1}\left(\tilde{c}_{2}^{1}\right)=(1-y) \tilde{c}^{0}, & \left.\partial_{2}\left(\tilde{c}^{3}\right)=(1-x) \tilde{c}_{2}^{2}\right)=(1+x y) \tilde{c}_{1}^{1}+(x-1) \tilde{c}_{2}^{1} .
\end{array}
$$

3.5 Proposição: O complexo de cadeias (3.3) é semi-exato.

Prova: A fim de calcularmos as composições $\partial_{i} \partial_{i+1}$, utilizaremos a notação matricial

$$
\left[\partial_{i} \partial_{i+1}\right]=\left[\partial_{i+1}\right]\left[\partial_{i}\right]
$$

onde cada linha da matriz corresponde à imagem de um elemento da base. Portanto,

$$
\begin{aligned}
{\left[\partial_{2} \partial_{3}\right] } & =\left[\begin{array}{ll}
1-x & 1-x y
\end{array}\right]\left[\begin{array}{cc}
-1-x & 1+y \\
1+x y & x-1
\end{array}\right] \\
& =\left[\begin{array}{ll}
-(1-x)(1+x)+(1-x y)(1+x y) & (1-x)(1+y)+(1-x y)(x-1)
\end{array}\right] \\
& =\left[\begin{array}{ll}
x^{2}-1+1-(x y)^{2} & 1-x+y-x y+x-x y x-1+x y
\end{array}\right] \\
& =\left[\begin{array}{ll}
0 & 0
\end{array}\right],
\end{aligned}
$$

pois $x^{2}=y^{2}=(x y)^{2}$ e $x y x=y$. Analogamente,

$$
\begin{aligned}
{\left[\partial_{1} \partial_{2}\right] } & =\left[\begin{array}{cc}
-1-x & 1+y \\
1+x y & x-1
\end{array}\right]\left[\begin{array}{l}
x-1 \\
y-1
\end{array}\right] \\
& =\left[\begin{array}{c}
-(1+x)(x-1)+(1+y)(y-1) \\
(1+x y)(x-1)+(x-1)(y-1)
\end{array}\right] \\
& =\left[\begin{array}{c}
x-x^{2}+1+y^{2}-1 \\
x-1+x y x-x y+x y-x-y+1
\end{array}\right] \\
& =\left[\begin{array}{l}
0 \\
0
\end{array}\right] .
\end{aligned}
$$

A fim de visualizarmos a região fundamental como um todo e as três células de seu bordo, utilizamos na Figura 3.3 segmentos ao invés de arcos. 


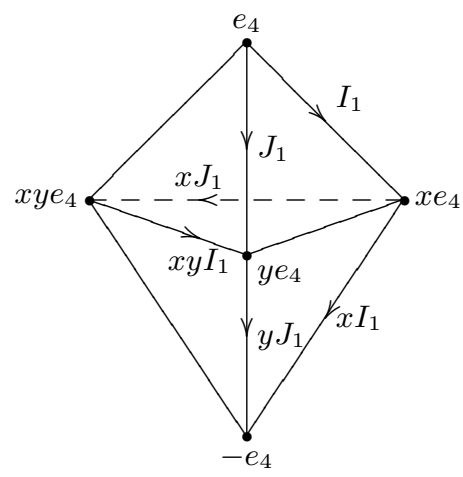

(a) 3-célula $\gamma$
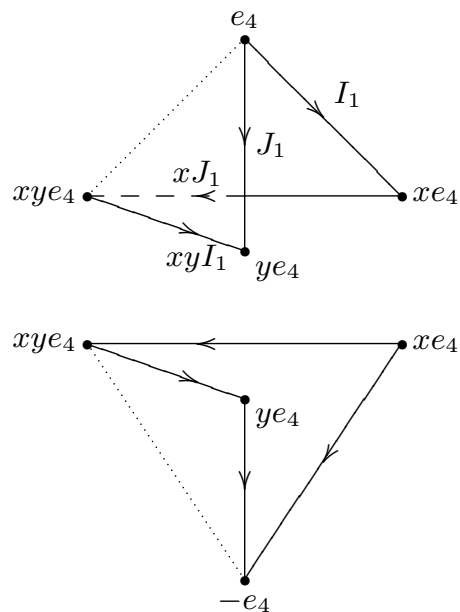

(b) 2-células $\sigma_{2}, \sigma_{3}$

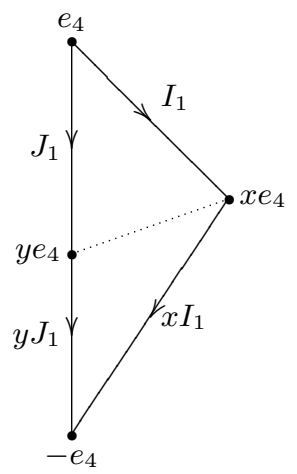

(c) 2-célula $\sigma_{1}$

Figura 3.3: Decomposição celular da região fundamental $\mathcal{F}_{8,3}$.

\subsubsection{Ação de $\mathcal{Q}_{4 t}$ em $S^{3}$}

Nesta seção, trabalharemos com o grupo $\mathcal{Q}_{4 t}$ e construiremos a região fundamental $\mathcal{F}_{4 t, 3}$, generalizando assim o processo da seção anterior. O leitor perceberá que isto ocorre naturalmente, com poucas modificações. Lembramos que $\theta=2 \pi / 2 t=\pi / t$ e $\zeta=\cos \theta+i \operatorname{sen} \theta \in \mathbb{C}$ é uma raíz $2 t$-ésima da unidade, ou seja, $\zeta^{2 t}=1$.

Definimos $\alpha: \mathcal{Q}_{4 t} \rightarrow U(2, \mathbb{C})$ por

$$
\alpha(x)=\left(\begin{array}{cc}
\zeta & 0 \\
0 & \bar{\zeta}
\end{array}\right) \quad \text { e } \quad \alpha(y)=\left(\begin{array}{cc}
0 & -1 \\
1 & 0
\end{array}\right) .
$$

Como anteriormente, calculamos

$$
\begin{array}{ll}
\alpha(x)(1,0)=(\zeta, 0) \equiv(\cos \theta, \operatorname{sen} \theta, 0,0) \in \mathbb{R}^{4} & \alpha(y)(1,0)=(0,1) \equiv(0,0,1,0) \in \mathbb{R}^{4} \\
\alpha(x)(i, 0)=(i \zeta, 0) \equiv(-\operatorname{sen} \theta, \cos \theta, 0,0) \in \mathbb{R}^{4} & \alpha(y)(i, 0)=(0, i) \equiv(0,0,0,1) \in \mathbb{R}^{4} \\
\alpha(x)(0,1)=(0, \bar{\zeta}) \equiv(0,0, \cos \theta,-\operatorname{sen} \theta) \in \mathbb{R}^{4} & \alpha(y)(0,1)=(-1,0) \equiv(-1,0,0,0) \in \mathbb{R}^{4} \\
\alpha(x)(0, i)=(0, i \bar{\zeta}) \equiv(0,0, \operatorname{sen} \theta, \cos \theta) \in \mathbb{R}^{4} & \alpha(y)(0, i)=(-i, 0) \equiv(0,-1,0,0) \in \mathbb{R}^{4}
\end{array}
$$

ou seja,

$$
\alpha(x) \equiv\left(\begin{array}{cccc}
\cos \theta & -\operatorname{sen} \theta & & \\
\operatorname{sen} \theta & \cos \theta & & \\
& & \cos \theta & \operatorname{sen} \theta \\
& & -\operatorname{sen} \theta & \cos \theta
\end{array}\right) \quad \text { e } \quad \alpha(y) \equiv\left(\begin{array}{cccc} 
& -1 & 0 \\
& 0 & -1 \\
1 & 0 & & \\
0 & 1 & &
\end{array}\right)
$$


Como na Observação 3.4, $x$ representa uma rotação de $\theta=\pi / t$ no sentido anti-horário (resp. horário) no plano-12 (resp. plano-34), tornando-os invariantes. A ação de y é a mesma. Notemos que $x^{k} e_{4}=e_{3}$ se, e somente se, $k=t / 2 \in \mathbb{Z}$, ou seja, $t \equiv 0 \bmod 2$.

A região fundamental $\mathcal{F}_{4 t, 3}$ pode ser vista na Figura 3.4. Posteriormente, escolheremos as células fundamentais de modo a construirmos um complexo de cadeias, como feito na seção anterior. O ângulo entre $J_{1}$ e $K_{1}$ é $\theta=\pi / t$, assim como os comprimentos dos arcos de referência $I_{1}, x I_{1}, \ldots, x^{t-1} I_{1}, x y I_{1}$. Os $\operatorname{arcos} J_{1}, K_{1}, y J_{1}, x y K_{1}$ medem $\pi / 2$, já que ligam (a menos de sentido) $\pm e_{4}$ ao "equador" da $S^{3}$, ou seja, a esfera $S^{2}$ da Figura 3.1.
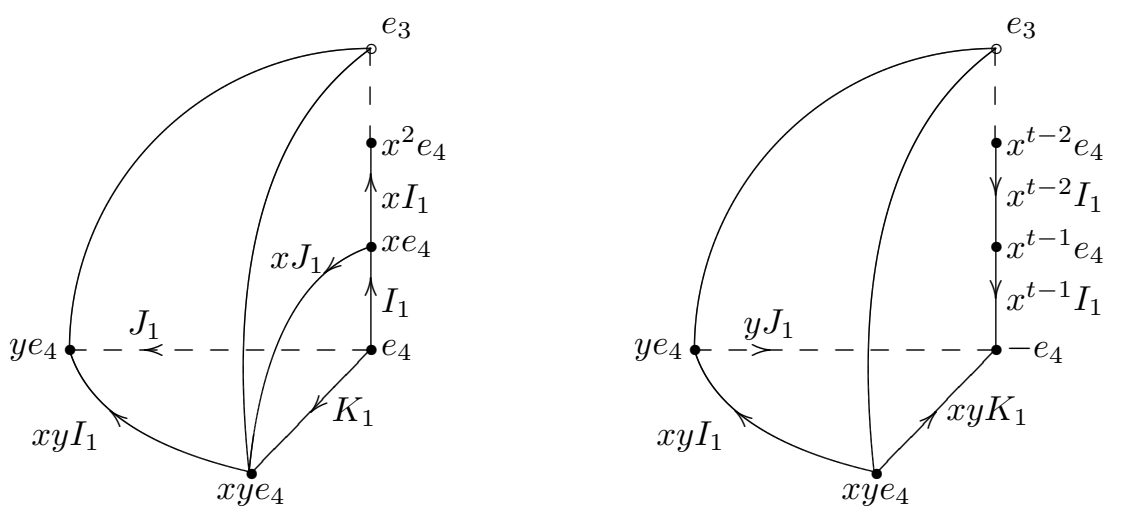

Figura 3.4: Região fundamental $\mathcal{F}_{4 t, 3}$.

Representando o plano-12 e o plano-34 separadamente, a posição dos arcos de referência utilizados na região fundamental pode ser vista na Figura 3.5 (perceba a troca dos planos feita pela ação de $x y)$.

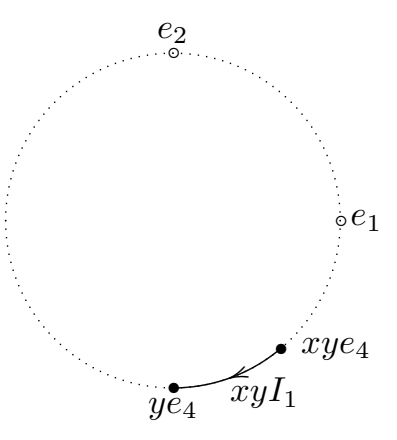

(a) plano-12

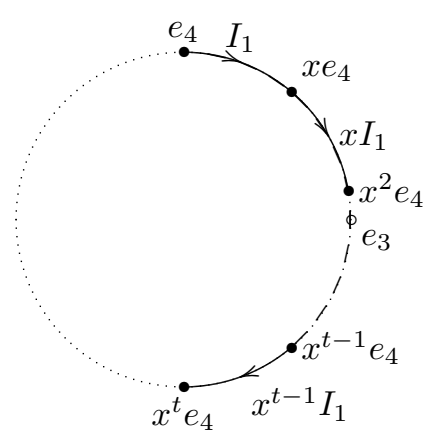

(b) plano-34

Figura 3.5: Arcos de referência da região fundamental $\mathcal{F}_{4 t, 3}$.

Denotando por $L_{x}=1+x+\cdots+x^{2 t-1}$ e $N_{x}=1+x+\cdots+x^{t-1}$, segue que

$$
\mathcal{F}_{4 t, 3}=x y I_{1} \tilde{*} N_{x} I_{1}
$$


Note que não há intersecção entre tal região e sua imagem pela ação de qualquer elemento não trivial do grupo, exceto pelos pontos do bordo, pois um ponto está no interior de uma região se pertence ao interior de um arco cujos bordos são pontos interiores dos arcos de referência de tal região, ou seja, dos arcos utilizados para o 'curved join'.

3.6 OBSERVAÇÃO: Para o grupo $\mathcal{Q}_{16}$, a Figura 3.6 mostra a ação de cada um dos 16 elementos. Pode-se ver o movimento de rotação realizado pelos elementos da forma $x^{k}$ (coluna esquerda) e $x^{k} y$ (coluna direita).

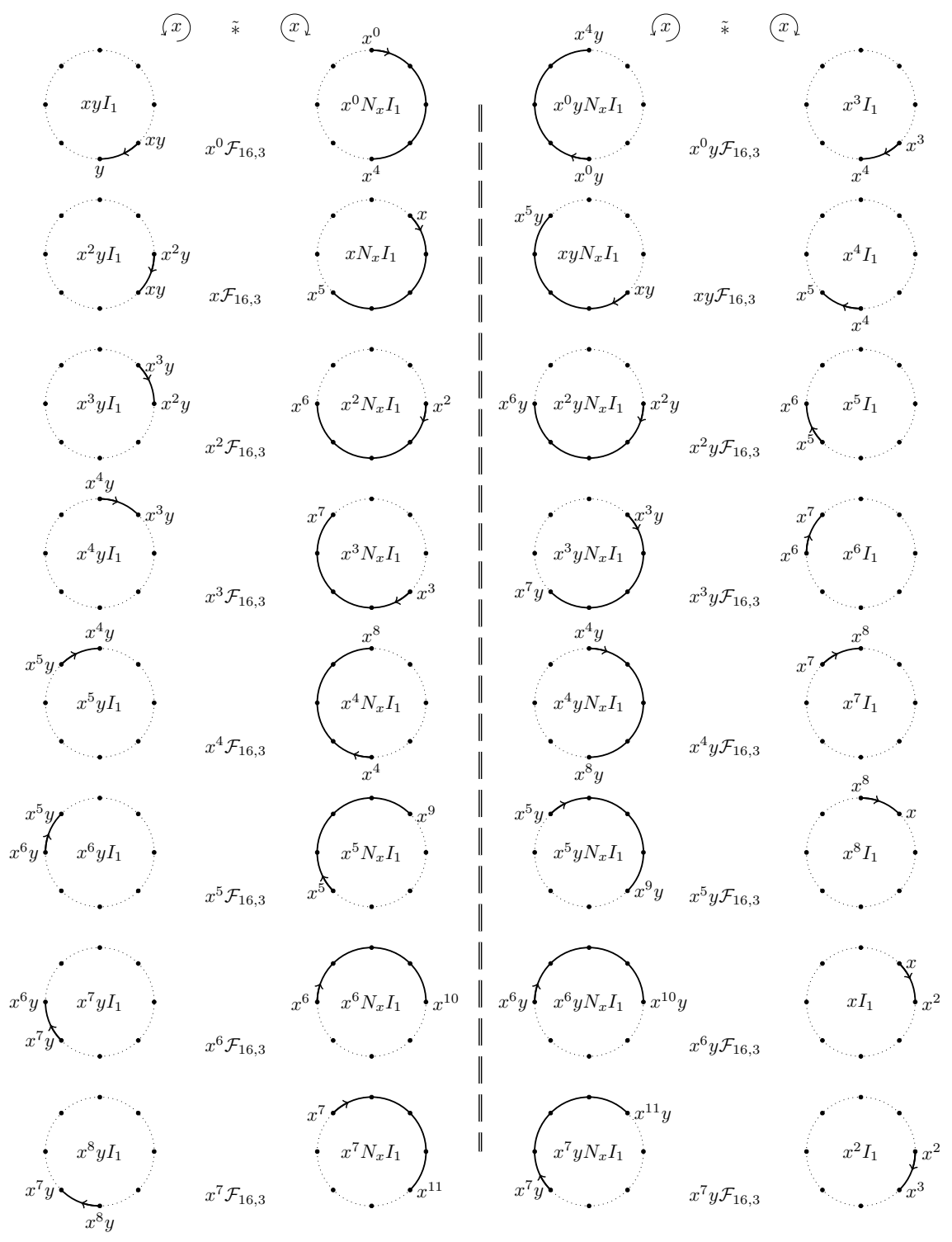

Figura 3.6: Ação de $\mathcal{Q}_{16}$ em $\mathcal{F}_{16,3}$. 
Com a notação da Seção 3.3.2, obtemos o complexo de cadeias semi-exato (cuja prova é análoga à prova de 3.5 , apenas usando a relação $\left.x^{t}=y^{2}=(x y)^{2}\right)$

$$
0 \longrightarrow \mathbb{Z}\left[\mathcal{Q}_{4 t}\right]\langle\gamma\rangle \stackrel{\partial_{3}}{\longrightarrow} \mathbb{Z}\left[\mathcal{Q}_{4 t}\right]\left\langle\sigma_{1}, \sigma_{2}\right\rangle \stackrel{\partial_{2}}{\longrightarrow} \mathbb{Z}\left[\mathcal{Q}_{4 t}\right]\left\langle I_{1}, J_{1}\right\rangle \stackrel{\partial_{1}}{\longrightarrow} \mathbb{Z}\left[\mathcal{Q}_{4 t}\right]\left\langle e_{4}\right\rangle \longrightarrow 0
$$

com

$$
\begin{aligned}
& \partial_{1}\left(I_{1}\right)=(x-1) e_{4}, \quad \partial_{2}\left(\sigma_{1}\right)=-N_{x} I_{1}+(1+y) J_{1}, \quad \partial_{3}(\gamma)=(1-x) \sigma_{1}+(1-x y) \sigma_{2}, \\
& \partial_{1}\left(J_{1}\right)=(y-1) e_{4}, \quad \partial_{2}\left(\sigma_{2}\right)=(1+x y) I_{1}+(x-1) J_{1} .
\end{aligned}
$$

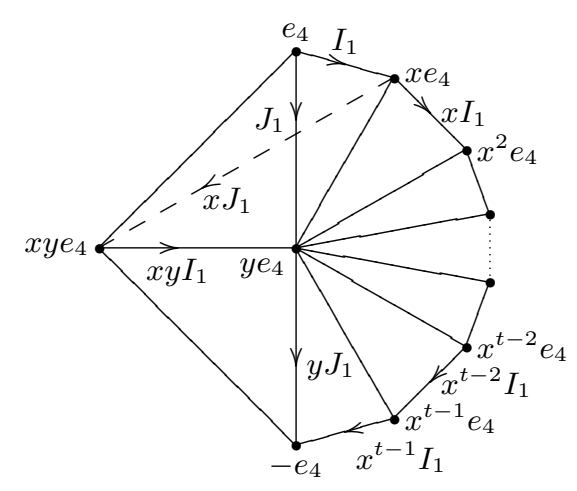

(a) 3-célula $\gamma$

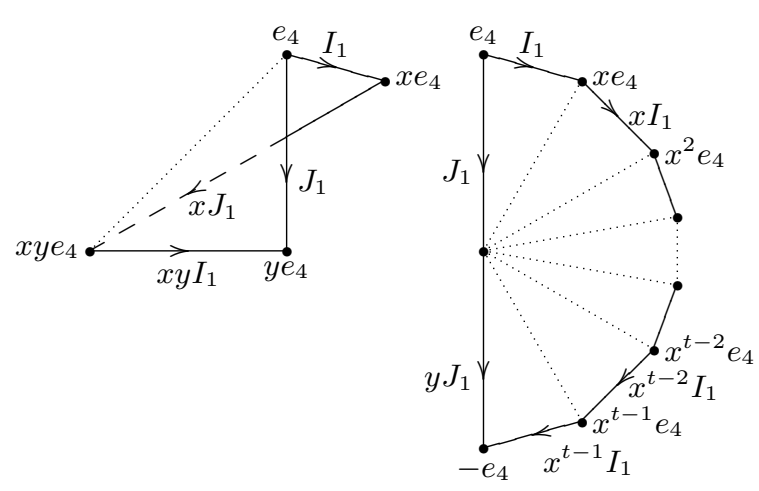

(b) 2-células $\sigma_{2}, \sigma_{1}$

Figura 3.7: Decomposição celular da região fundamental $\mathcal{F}_{4 t, 3}$.

\subsubsection{Ação de $\mathcal{Q}_{16}$ em $S^{7}$}

A fim de construirmos a região fundamental $\mathcal{F}_{4 t, 4 n-1}$, apresentamos aqui mais um caso particular, diferenciado dos anteriores pela dimensão da esfera. O fato de trabalharmos agora em $\mathbb{R}^{8}$ nos leva a perceber uma periodicidade no complexo de cadeias, que será concretizada no caso geral.

Seja $\Sigma_{2}=L_{x} I_{1}$ uma cópia de $S^{1}$ no plano-34 possuindo uma decomposição celular com $2 t$ 0 -células $\left\{e_{4}, x e_{4}, \ldots, x^{2 t-1} e_{4}\right\}$ e com $2 t$-células $\left\{I_{1}, x I_{1}, \ldots, x^{2 t-1} I_{1}\right\}$. Note que $\Sigma_{1}=x y \Sigma_{2}$ está no plano-12 e que $x y \Sigma_{2} \tilde{*} \Sigma_{2}$ é uma 3 -esfera, que pode ser obtida por

$$
x y \Sigma_{2} \tilde{*} \Sigma_{2}=P_{x, y}\left(x y I_{1} \tilde{*} N_{x} I_{1}\right)
$$

onde $P_{x, y}=L_{x}(1+y)$. A região fundamental será

$$
\mathcal{F}_{16,7}=x y \Sigma_{2} \tilde{*} \Sigma_{2} \tilde{*} x y I_{2} \tilde{*} N_{x} I_{2}
$$




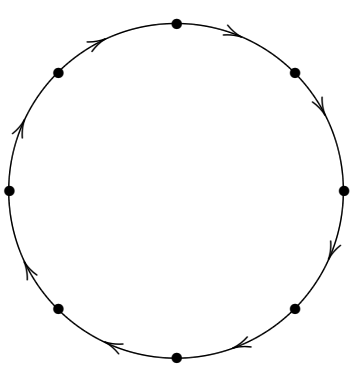

(a) $x y \Sigma_{2}$ no plano-12

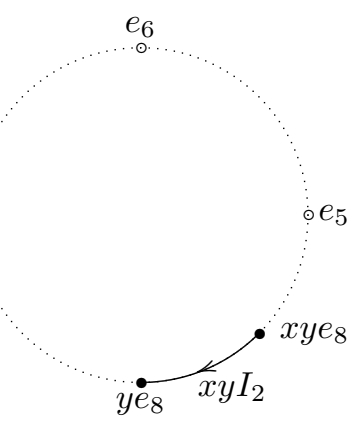

(c) $x y I_{2}$ no plano-56

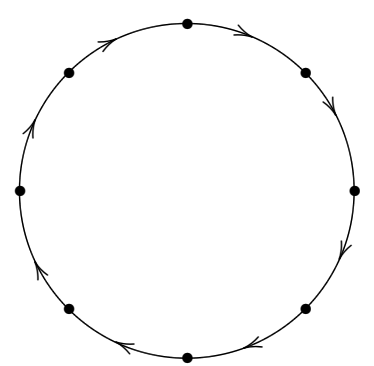

(b) $\Sigma_{2}$ no plano-34

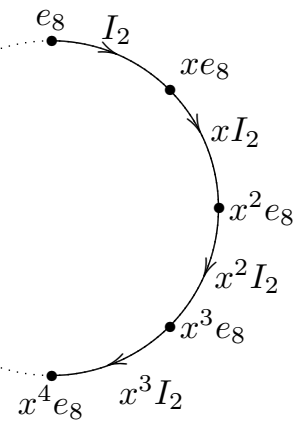

(d) $N_{x} I_{2}$ no plano- 78

Figura 3.8: Arcos de referência da região fundamental $\mathcal{F}_{16,7}$.

onde $\gamma^{\prime}=x y I_{2} \tilde{*} N_{x} I_{2}$ se comporta como $\gamma$ na região $\mathcal{F}_{4 t, 3}$, permitindo o uso das decomposições já obtidas na Seção 3.3.3. A situação torna-se mais clara com o auxílio da Figura 3.8.

As $k$-células $c_{i}^{k}$ são

$$
\begin{array}{rlrl}
c^{7} & =x y \Sigma_{2} \tilde{*} \Sigma_{2} \tilde{*} x y I_{2} \tilde{*} N_{x} I_{2} & \\
c_{1}^{6}=x y \Sigma_{2} \tilde{*} \Sigma_{2} \tilde{*} y e_{8} \tilde{*} N_{x} I_{2} & c_{3}^{6}=x y \Sigma_{2} \tilde{*} \Sigma_{2} \tilde{*} x y I_{2} \tilde{*} x^{t} e_{8} \\
c_{2}^{6}=x y \Sigma_{2} \tilde{*} \Sigma_{2} \tilde{*} x y e_{8} \tilde{*} N_{x} I_{2} & c_{4}^{6}=x y \Sigma_{2} \tilde{*} \Sigma_{2} \tilde{*} x y I_{2} \tilde{*} x^{0} e_{8} \\
c_{1}^{5}=x y \Sigma_{2} \tilde{*} \Sigma_{2} \tilde{*} \emptyset \tilde{*} N_{x} I_{2} & c_{2}^{5}=x y \Sigma_{2} \tilde{*} \Sigma_{2} \tilde{*} x y I_{2} \tilde{*} \emptyset \\
c_{1}^{4}=x y \Sigma_{2} \tilde{*} \Sigma_{2} \tilde{*} \emptyset \tilde{*} x^{t} e_{8} & c_{3}^{4}=x y \Sigma_{2} \tilde{*} \Sigma_{2} \tilde{*} y e_{8} \tilde{*} \emptyset \\
c_{2}^{4}=x y \Sigma_{2} \tilde{*} \Sigma_{2} \tilde{*} \emptyset \tilde{*} x^{0} e_{8} & c_{4}^{4}=x y \Sigma_{2} \tilde{*} \Sigma_{2} \tilde{*} x y e_{8} \tilde{*} \emptyset \\
c^{3}=x y \Sigma_{2} \tilde{*} \Sigma_{2} & \\
c_{1}^{2}=y e_{4} \tilde{*} N_{x} I_{1} & c_{3}^{2}=x y I_{1} \tilde{*} x^{t} e_{4} \\
c_{2}^{2}=x y e_{4} \tilde{*} N_{x} I_{1} & c_{4}^{2}=x y I_{1} \tilde{*} x^{0} e_{4} \\
c_{1}^{1}=N_{x} I_{1} & c_{2}^{1}=x y I_{1} \\
c_{1}^{0}=x^{t} e_{4} & c_{3}^{0}=y e_{4} \\
c_{2}^{0}=x^{0} e_{4} & c_{4}^{0}=x y e_{4}
\end{array}
$$


Escolhemos então as $k$-células fundamentais $\tilde{c}_{i}^{k}$, onde $\sigma_{i}^{\prime}$ se comporta como $\sigma_{i}$.

$$
\begin{array}{ll}
\tilde{c}^{7}=x y \Sigma_{2} \tilde{*} \Sigma_{2} \tilde{*} \gamma^{\prime} & \\
\tilde{c}_{1}^{6}=x y \Sigma_{2} \tilde{*} \Sigma_{2} \tilde{*} \sigma_{1}^{\prime} & \tilde{c}_{2}^{6}=x y \Sigma_{2} \tilde{*} \Sigma_{2} \tilde{*} \sigma_{2}^{\prime} \\
\tilde{c}_{1}^{5}=x y \Sigma_{2} \tilde{*} \Sigma_{2} \tilde{*} I_{2} & \tilde{c}_{2}^{5}=x y \Sigma_{2} \tilde{*} \Sigma_{2} \tilde{*} J_{2} \\
\tilde{c}^{4}=x y \Sigma_{2} \tilde{*} \Sigma_{2} \tilde{*} e_{8} & \\
\tilde{c}^{3}=x y I_{1} \tilde{*} N_{x} I_{1} & \\
\tilde{c}_{1}^{2}=\sigma_{1} & \tilde{c}_{2}^{2}=\sigma_{2} \\
\tilde{c}_{1}^{1}=I_{1} & \tilde{c}_{2}^{1}=J_{1} \\
\tilde{c}^{0}=e_{4} &
\end{array}
$$

Resumindo, para $I_{1}=\left[e_{4}, x e_{4}\right], J_{1}=\left[e_{4}, y e_{4}\right], I_{2}=\left[e_{8}, x e_{8}\right]$ e $J_{2}=\left[e_{8}, y e_{8}\right]$ temos as cadeias

$$
\begin{array}{ll}
C_{7}\left(\mathcal{F}_{4 t, 7}\right)=\left\langle\tilde{c}^{7}\right\rangle & C_{3}\left(\mathcal{F}_{4 t, 7}\right)=\left\langle\tilde{c}^{3}\right\rangle \\
C_{6}\left(\mathcal{F}_{4 t, 7}\right)=\left\langle\tilde{c}_{1}^{6}, \tilde{c}_{2}^{6}\right\rangle & C_{2}\left(\mathcal{F}_{4 t, 7}\right)=\left\langle\tilde{c}_{1}^{2}, \tilde{c}_{2}^{2}\right\rangle \\
C_{5}\left(\mathcal{F}_{4 t, 7}\right)=\left\langle\tilde{c}_{1}^{5}, \tilde{c}_{2}^{5}\right\rangle & C_{1}\left(\mathcal{F}_{4 t, 7}\right)=\left\langle\tilde{c}_{1}^{1}, \tilde{c}_{2}^{1}\right\rangle \\
C_{4}\left(\mathcal{F}_{4 t, 7}\right)=\left\langle\tilde{c}^{4}\right\rangle & C_{0}\left(\mathcal{F}_{4 t, 7}\right)=\left\langle\tilde{c}^{0}\right\rangle
\end{array}
$$

e os operadores bordo são dados por (notando que $\partial_{4}=P_{x, y}$ devido à equação (3.4))

$$
\begin{aligned}
{\left[\partial_{7}\right]=\left[\partial_{3}\right] } & =\left[\begin{array}{cc}
1-x & 1-x y
\end{array}\right] \\
{\left[\partial_{6}\right]=\left[\partial_{2}\right] } & =\left[\begin{array}{cc}
-N_{x} & 1+y \\
1+x y & x-1
\end{array}\right] \\
{\left[\partial_{5}\right]=\left[\partial_{1}\right] } & =\left[\begin{array}{c}
x-1 \\
y-1
\end{array}\right] \\
{\left[\partial_{4}\right] } & =\left[P_{x, y}\right]
\end{aligned}
$$

3.7 ProposiçÃo: Nas condições acima, o complexo de cadeias (3.5) é semi-exato.

$$
\begin{aligned}
& 0 \longrightarrow \mathbb{Z}\left[\mathcal{Q}_{4 t}\right]\left\langle\tilde{c}^{7}\right\rangle \stackrel{\partial_{7}}{\longrightarrow} \mathbb{Z}\left[\mathcal{Q}_{4 t}\right]\left\langle\tilde{c}_{1}^{6}, \tilde{c}_{2}^{6}\right\rangle \stackrel{\partial_{6}}{\longrightarrow} \mathbb{Z}\left[\mathcal{Q}_{4 t}\right]\left\langle\tilde{c}_{1}^{5}, \tilde{c}_{2}^{5}\right\rangle \stackrel{\partial_{5}}{\longrightarrow} \mathbb{Z}\left[\mathcal{Q}_{4 t}\right]\left\langle\tilde{c}^{4}\right\rangle \stackrel{\partial_{4}}{\longrightarrow} \\
& \stackrel{\partial_{4}}{\longrightarrow} \mathbb{Z}\left[\mathcal{Q}_{4 t}\right]\left\langle\tilde{c}^{3}\right\rangle \stackrel{\partial_{3}}{\longrightarrow} \mathbb{Z}\left[\mathcal{Q}_{4 t}\right]\left\langle\tilde{c}_{1}^{2}, \tilde{c}_{2}^{2}\right\rangle \stackrel{\partial_{2}}{\longrightarrow} \mathbb{Z}\left[\mathcal{Q}_{4 t}\right]\left\langle\tilde{c}_{1}^{1}, \tilde{c}_{2}^{1}\right\rangle \stackrel{\partial_{1}}{\longrightarrow} \mathbb{Z}\left[\mathcal{Q}_{4 t}\right]\left\langle\tilde{c}^{0}\right\rangle \stackrel{\longrightarrow}{\longrightarrow} 0
\end{aligned}
$$


PROVA: De modo semelhante à prova de 3.5, temos

$$
\begin{aligned}
{\left[\partial_{6} \partial_{7}\right] } & =\left[\begin{array}{cc}
1-x & 1-x y
\end{array}\right]\left[\begin{array}{cc}
-N_{x} & 1+y \\
1+x y & x-1
\end{array}\right] \\
& =\left[\begin{array}{ll}
-(1-x) N_{x}+(1-x y)(1+x y) & (1-x)(1+y)+(1-x y)(x-1)
\end{array}\right] \\
& =\left[\begin{array}{ll}
x^{t}-1+1-(x y)^{2} & 1-x+y-x y+x-x y x-1+x y
\end{array}\right] \\
& =\left[\begin{array}{ll}
0 & 0
\end{array}\right]=\left[\partial_{2} \partial_{3}\right]
\end{aligned}
$$

pois $x^{t}=y^{2}=(x y)^{2}$ e $x y x=y$. Também,

$$
\begin{aligned}
& {\left[\partial_{5} \partial_{6}\right]=\left[\begin{array}{cc}
-N_{x} & 1+y \\
1+x y & x-1
\end{array}\right]\left[\begin{array}{l}
x-1 \\
y-1
\end{array}\right]} \\
& =\left[\begin{array}{c}
-N_{x}(x-1)+(1+y)(y-1) \\
(1+x y)(x-1)+(x-1)(y-1)
\end{array}\right] \\
& =\left[\begin{array}{c}
-x^{t}+1+y^{2}-1 \\
x-1+x y x-x y+x y-x-y+1
\end{array}\right] \\
& =\left[\begin{array}{l}
0 \\
0
\end{array}\right]=\left[\partial_{1} \partial_{2}\right] \text {, } \\
& {\left[\partial_{4} \partial_{5}\right]=\left[\begin{array}{l}
x-1 \\
y-1
\end{array}\right]\left[P_{x, y}\right]} \\
& =\left[\begin{array}{l}
(x-1) L_{x}(1+y) \\
(y-1) L_{x}(1+y)
\end{array}\right] \\
& =\left[\begin{array}{l}
0 \\
0
\end{array}\right]=\left[\partial_{0} \partial_{1}\right] \text {, } \\
& {\left[\partial_{3} \partial_{4}\right]=\left[P_{x, y}\right]\left[\begin{array}{ll}
1-x & 1-x y
\end{array}\right]} \\
& =\left[\begin{array}{ll}
P_{x, y}(1-x) & P_{x, y}(1-x y)
\end{array}\right] \\
& =\left[\begin{array}{ll}
0 & 0
\end{array}\right] \text {, }
\end{aligned}
$$

pois

$$
\begin{aligned}
P_{x, y}(1-x y) & =\left(1+x+\cdots+x^{2 t-2}+x^{2 t-1}\right)+\underbrace{\left(y+x y+\cdots+x^{2 t-1} y\right)}_{a} \\
& -\underbrace{\left(x y+x^{2} y+\cdots+x^{2 t-1} y+x^{2 t} y\right)}_{a}-\left(y x y+(x y)^{2}+\cdots+x^{2 t-2}(x y)^{2}\right) \\
& =\left(1+x+\cdots+x^{2 t-2}+x^{2 t-1}\right)-\left(y x y+(x y)^{2}+\cdots+x^{2 t-2}(x y)^{2}\right) \\
& =\left(1+x+\cdots+x^{2 t-2}+x^{2 t-1}\right)-\left(x^{t-1}+x^{t}+\cdots+x^{t-2}\right) \\
& =0
\end{aligned}
$$




$$
\begin{aligned}
P_{x, y}(1-x)=(1+x+\cdots+ & \left.x^{2 t-2}+x^{2 t-1}\right)+\left(y+x y+\cdots+x^{2 t-1} y\right) \\
& -\left(x+x^{2}+\cdots+x^{2 t-1}+x^{2 t}\right)-\left(y x+x y x+\cdots+x^{2 t-1} y x\right)=0
\end{aligned}
$$

devido à $y x=x^{-1} y=x^{2 t-1} y$.

\subsubsection{Ação de $\mathcal{Q}_{4 t}$ em $S^{4 n-1}$}

Com as notações da Seção 3.3.1, a ação do grupo $\mathcal{Q}_{4 t}$ em $S^{4 n-1}$ se dá através da representação

$$
\alpha=\alpha_{q_{1}} \oplus \alpha_{q_{2}} \oplus \cdots \oplus \alpha_{q_{n}}: \mathcal{Q}_{4 t} \rightarrow U(2 n, \mathbb{C})
$$

onde $\alpha_{q_{j}}: \mathcal{Q}_{4 t} \rightarrow U(2, \mathbb{C})$ é dada por

$$
\alpha_{q_{j}}(x)=\left(\begin{array}{cc}
\zeta^{q_{j}} & 0 \\
0 & \zeta^{-q_{j}}
\end{array}\right) \quad \text { e } \quad \alpha_{q_{j}}(y)=\left(\begin{array}{cc}
0 & 1 \\
-1 & 0
\end{array}\right) .
$$

Tal representação faz com que a ação de $x$ seja uma rotação de $q_{j} \theta$ no sentido horário (resp. anti-horário) em $\Sigma_{2 j}$ (resp. $\Sigma_{2 j-1}$ ). A ação de $y$ é como descrita anteriormente. Todos os casos particulares feitos anteriormente podem ser obtidos tomando $q_{j}=1$ e conseqüentemente, $r_{j}=1$.

Assim sendo, a região fundamental é

$$
\mathcal{F}_{4 t, 4 n-1}=\Sigma_{1} \tilde{*} \Sigma_{2} \tilde{*} \cdots \tilde{*} \Sigma_{2 n-3} \tilde{*} \Sigma_{2 n-2} \tilde{*} x^{r_{n}} y I_{n} \tilde{*} N_{x^{r_{n}}} I_{n}
$$

cujas células fundamentais são

$$
\begin{gathered}
\tilde{c}^{4 k-1}=\Sigma^{2 k-2} \tilde{*} \gamma^{\prime}, \quad \tilde{c}_{1}^{4 k-2}=\Sigma^{2 k-2} \tilde{*} \sigma_{1}^{\prime}, \quad \tilde{c}_{1}^{4 k-3}=\Sigma^{2 k-2} \tilde{*} I_{k}, \quad \tilde{c}^{4 k-4}=\Sigma^{2 k-2} \tilde{*} v_{k}, \\
\tilde{c}_{2}^{4 k-2}=\Sigma^{2 k-2} \tilde{*} \sigma_{2}^{\prime}, \quad \tilde{c}_{2}^{4 k-3}=\Sigma^{2 k-2} \tilde{*} J_{k},
\end{gathered}
$$

o complexo de cadeias sobre $\mathbb{Z}\left[\mathcal{Q}_{4 t}\right]$ é

$$
\begin{aligned}
\cdots \stackrel{Z}{\longrightarrow}\left[\mathcal{Q}_{4 t}\right]\left\langle\tilde{c}^{4 k-1}\right\rangle \stackrel{\partial_{4 k-1}}{\longrightarrow} \mathbb{Z}\left[\mathcal{Q}_{4 t}\right]\left\langle\tilde{c}_{1}^{4 k-2}, \tilde{c}_{2}^{4 k-2}\right\rangle \stackrel{\partial_{4 k-2}}{\longrightarrow} \\
\stackrel{\partial_{4 k-2}}{\longrightarrow} \mathbb{Z}\left[\mathcal{Q}_{4 t}\right]\left\langle\tilde{c}_{1}^{4 k-3}, \tilde{c}_{2}^{4 k-3}\right\rangle \stackrel{\partial_{4 k-3}}{\longrightarrow} \mathbb{Z}\left[\mathcal{Q}_{4 t}\right]\left\langle\tilde{c}^{4 k-4}\right\rangle \stackrel{\partial_{4 k-4}}{\longrightarrow} \cdots
\end{aligned}
$$

com operadores bordo dados por

$$
\begin{aligned}
& {\left[\begin{array}{ll}
\partial_{4 k-1}
\end{array}\right]=\left[\begin{array}{ll}
1-x^{r_{k}} & 1-x^{r_{k}} y
\end{array}\right], \quad\left[\partial_{4 k-2}\right]=\left[\begin{array}{cc}
-N_{x^{r_{k}}} & 1+y \\
1+x^{r_{k}} y & x^{r_{k}}-1
\end{array}\right],} \\
& {\left[\partial_{4 k-3}\right]=\left[\begin{array}{c}
x^{r_{k}}-1 \\
y-1
\end{array}\right], \quad\left[\partial_{4 k-4}\right]=\left[P_{x^{r_{k}, y}}\right],}
\end{aligned}
$$

para $1 \leq k \leq n$ (exceto $\partial_{0}=0$ ). O uso de $x^{r_{k}}$ é necessário pois o vértice adjacente (no sentido horário) a $v_{k} \in \Sigma_{2 k}$ é exatamente $x^{r_{k}} v_{k}$, já que $r_{k} q_{k} \equiv 1 \bmod 2 t$.

Observe que a prova de 3.5 se aplica ao complexo (3.7), apenas trocando $x$ por $x^{r_{k}}$, mostrando então que tal complexo é semi-exato. Podemos então introduzir o espaço quociente. 
3.8 Definição (Forma espacial esférica quaterniônica): Seja $\mathcal{Q}_{4 t}$ o grupo dos quatérnios generalizado de ordem $4 t$. Sejam $q_{1}, \ldots, q_{n}$ inteiros (não necessariamente distintos) primos com $2 t$. Com a ação de $\mathcal{Q}_{4 t}$ em $S^{4 n-1}$ descrita acima, formamos o espaço quociente

$$
\mathcal{Q}\left(2 t ; q_{1}, \ldots, q_{n}\right)=S^{4 n-1} / \alpha\left(\mathcal{Q}_{4 t}\right)
$$

chamado de forma espacial esférica quaterniônica.

3.9 LEMA: Qualquer forma espacial esférica quaterniônica é homeomorfa a uma forma como em (3.8), $\operatorname{com} 1 \leq q_{1} \leq q_{2} \leq \ldots \leq q_{n}<t$.

Prova: Primeiramente, $\mathcal{Q}\left(2 t ; q_{1}, \ldots, q_{l}, \ldots, q_{n}\right) \approx \mathcal{Q}\left(2 t ; q_{1}, \ldots,-q_{l}, \ldots, q_{n}\right)$. De fato, considerando $f: S^{4 n-1} \rightarrow S^{4 n-1}$ o homeomorfismo definido por $f\left(z_{1}, \ldots, z_{2 n}\right)=\left(\bar{z}_{1}, \ldots, \bar{z}_{2 n}\right)$, vemos que $f$ é compatível com as ações $\alpha_{1}$ e $\alpha_{2}$ (a ação de $y$ é a mesma nos dois casos) dadas por

$$
\begin{aligned}
& \left(\alpha_{1}(x)\right)\left(\ldots, z_{2 l-1}, z_{2 l}, \ldots\right)=\left(\ldots, \zeta^{q_{l}} z_{2 l-1}, \zeta^{-q_{l}} z_{2 l}, \ldots\right), \\
& \left(\alpha_{2}(x)\right)\left(\ldots, z_{2 l-1}, z_{2 l}, \ldots\right)=\left(\ldots, \zeta^{-q_{l}} z_{2 l-1}, \zeta^{q_{l}} z_{2 l}, \ldots\right),
\end{aligned}
$$

definindo assim os espaços $\mathcal{Q}\left(2 t ; \ldots, q_{l}, \ldots, q_{s}, \ldots\right)$ e $\mathcal{Q}\left(2 t ; \ldots, q_{s}, \ldots, q_{l}, \ldots\right)$. Tomando o quociente, temos o homeomorfismo desejado.

Por fim, são homeomorfos $\mathcal{Q}\left(2 t ; \ldots, q_{l}, \ldots, q_{s}, \ldots\right) \approx \mathcal{Q}\left(2 t ; \ldots, q_{s}, \ldots, q_{l}, \ldots\right)$. De fato, seja $h: S^{4 n-1} \rightarrow S^{4 n-1}$ o homeomorfismo dado por

$$
h\left(\ldots, z_{2 l-1}, z_{2 l}, \ldots, z_{2 s-1}, z_{2 s}, \ldots\right)=\left(\ldots, z_{2 s-1}, z_{2 s}, \ldots, z_{2 l-1}, z_{2 l}, \ldots\right)
$$

Como acima, $h$ também é compatível com as ações

$$
\begin{aligned}
& \left(\alpha_{1}(x)\right)\left(\ldots, z_{2 l-1}, z_{2 l}, \ldots, z_{2 s-1}, z_{2 s}, \ldots\right)=\left(\ldots, \zeta^{q_{l}} z_{2 l-1}, \zeta^{-q_{l}} z_{2 l}, \ldots, \zeta^{q_{s}} z_{2 s-1}, \zeta^{-q_{s}} z_{2 s}, \ldots\right) \\
& \left(\alpha_{2}(x)\right)\left(\ldots, z_{2 l-1}, z_{2 l}, \ldots, z_{2 s-1}, z_{2 s}, \ldots\right)=\left(\ldots, \zeta^{q_{s}} z_{2 l-1}, \zeta^{-q_{s}} z_{2 l}, \ldots, \zeta^{q_{l}} z_{2 s-1}, \zeta^{-q_{l}} z_{2 s}, \ldots\right),
\end{aligned}
$$

e portanto, segue o resultado.

Os grupos de homologia: Aproveitamos o complexo (3.7) acima e calculamos os grupos de homologia de $\mathcal{Q}=\mathcal{Q}\left(2 t ; q_{1}, \ldots, q_{n}\right)$, com representações $\rho: \mathcal{Q}_{4 t} \rightarrow \mathbb{Z}_{2}$ (em particular, com coeficientes em $\mathbb{Z}$ ). As possíveis representações para $\mathcal{Q}_{4 t}$ devem preservar as relações do grupo, ou seja, devem satisfazer às equações

$$
\begin{aligned}
& \rho(x)^{t}=\rho\left(x^{t}\right)=\rho\left(y^{2}\right)=\rho(y)^{2}, \\
& \rho(x y x)=\rho(x) \rho(y) \rho(x)=\rho(y) .
\end{aligned}
$$


Seja $\rho_{0}:(x, y) \mapsto(1,1)$ a representação trivial. Os operadores bordo são dados por

$$
\begin{array}{lll}
{\left[\partial_{4 k-1}\right]=\left[\begin{array}{ll}
0 & 0
\end{array}\right],} & {\left[\partial_{4 k-2}\right]=\left[\begin{array}{cc}
-t & 2 \\
2 & 0
\end{array}\right],} \\
{\left[\partial_{4 k-3}\right]=\left[\begin{array}{l}
0 \\
0
\end{array}\right],} & {\left[\partial_{4 k-4}\right]=[4 t],}
\end{array}
$$

exceto por $\partial_{0}=0$, de onde segue que ker $\partial_{0} \simeq \mathbb{Z}$. Assim, obtemos os núcleos e imagens, dados por

\begin{tabular}{c|cccc} 
& $\partial_{4 k-1}$ & $\partial_{4 k-2}$ & $\partial_{4 k-3}$ & $\partial_{4 k-4 \neq 0}$ \\
\hline $\operatorname{ker}$ & $\mathbb{Z}$ & 0 & $\mathbb{Z} \oplus \mathbb{Z}$ & 0 \\
\hline $\operatorname{im}$ & 0 & $\begin{array}{r}2 \mathbb{Z} \oplus 2 \mathbb{Z} \\
\mathbb{Z} \oplus 4 \mathbb{Z}\end{array} \quad t$ ímpar & 0 & $4 t \mathbb{Z}$
\end{tabular}

O cálculo de $\operatorname{im}\left(\partial_{4 k-2}\right)$ merece atenção, pois necessitamos de algumas mudanças nos geradores, dependendo da paridade de $t$. Suponhamos $t$ par. Como

$$
\partial_{4 k-2}\left(\tilde{c}_{1}^{4 k-2}\right)=-t \tilde{c}_{1}^{4 k-3}+2 \tilde{c}_{2}^{4 k-3} \text { e } \partial_{4 k-2}\left(\tilde{c}_{2}^{4 k-2}\right)=2 \tilde{c}_{1}^{4 k-3}
$$

mudamos, por uma matriz de determinante 1 , o conjunto de geradores

$$
\left\{\tilde{c}_{1}^{4 k-2}, \tilde{c}_{2}^{4 k-2}\right\} \longrightarrow\left\{\tilde{c}_{1}^{4 k-2}+t / 2 \tilde{c}_{2}^{4 k-2}, \tilde{c}_{2}^{4 k-2}\right\}
$$

obtendo $2 \tilde{c}_{1}^{4 k-3}, 2 \tilde{c}_{2}^{4 k-3} \in \operatorname{im}\left(\partial_{4 k-2}\right)$. No entanto, $\tilde{c}_{1}^{4 k-3}, \tilde{c}_{2}^{4 k-3} \notin \operatorname{im}\left(\partial_{4 k-2}\right)$, pois para isto teríamos que obter solução em $\mathbb{Z}$ para os sistemas

$$
\left\{\begin{array} { r } 
{ - t x + 2 y = 0 } \\
{ 2 x = 1 }
\end{array} \text { e } \left\{\begin{array}{r}
-t x+2 y=1 \\
2 x=0
\end{array},\right.\right.
$$

o que não ocorre. Logo, $\operatorname{im}\left(\partial_{4 k-2}\right) \simeq 2 \mathbb{Z} \oplus 2 \mathbb{Z}$.

Suponhamos $t=2 k+1$ ímpar, para algum $k \in \mathbb{N}$. Mudamos então os geradores em dimensão $4 k-3$, ou seja,

$$
\left\{\tilde{c}_{1}^{4 k-3}, \tilde{c}_{2}^{4 k-3}\right\} \longrightarrow\left\{3 \tilde{c}_{1}^{4 k-3}+2 \tilde{c}_{2}^{4 k-3}, 4 \tilde{c}_{1}^{4 k-3}+3 \tilde{c}_{2}^{4 k-3}\right\}
$$

obtendo

$$
\begin{gathered}
\partial_{4 k-2}\left(\tilde{c}_{1}^{4 k-2}+(k+2) \tilde{c}_{2}^{4 k-2}\right)=3 \tilde{c}_{1}^{4 k-3}+2 \tilde{c}_{2}^{4 k-3}, \\
\partial_{4 k-2}\left(6 \tilde{c}_{1}^{4 k-2}+(8+3 t) \tilde{c}_{2}^{4 k-2}\right)=4\left(4 \tilde{c}_{1}^{4 k-3}+3 \tilde{c}_{2}^{4 k-3}\right) .
\end{gathered}
$$

Porém,

$$
4 \tilde{c}_{1}^{4 k-3}+3 \tilde{c}_{2}^{4 k-3}, 2\left(4 \tilde{c}_{1}^{4 k-3}+3 \tilde{c}_{2}^{4 k-3}\right), 3\left(4 \tilde{c}_{1}^{4 k-3}+3 \tilde{c}_{2}^{4 k-3}\right) \notin \operatorname{im}\left(\partial_{4 k-2}\right),
$$

por motivos análogos ao do caso anterior. Logo, $\operatorname{im}\left(\partial_{4 k-2}\right) \simeq \mathbb{Z} \oplus 4 \mathbb{Z}$.

Obtemos então os grupos de homologia com coeficientes em $\mathbb{Z}$,

$$
\begin{aligned}
H_{4 n-1}(\mathcal{Q} ; \mathbb{Z}) \simeq H_{0}(\mathcal{Q} ; \mathbb{Z}) \simeq \mathbb{Z}, & H_{4 k-1}(\mathcal{Q} ; \mathbb{Z}) \simeq \mathbb{Z}_{4 t}, \\
H_{4 k-2}(\mathcal{Q} ; \mathbb{Z})=H_{4 k-4}(\mathcal{Q} ; \mathbb{Z})=0, & H_{4 k-3}(\mathcal{Q} ; \mathbb{Z}) \simeq\left\{\begin{array}{cl}
\mathbb{Z}_{2} \oplus \mathbb{Z}_{2} & t \text { par } \\
\mathbb{Z}_{4} & t \text { ímpar }
\end{array} .\right.
\end{aligned}
$$


Seja $\rho_{x}:(x, y) \mapsto(-1,1)$ uma representação não trivial. Por $(3.9)$, apenas o caso $t$ par é permitido e os operadores bordo são

$$
\begin{array}{lll}
{\left[\partial_{4 k-1}\right]=\left[\begin{array}{cc}
2 & 2
\end{array}\right],} & {\left[\partial_{4 k-2}\right]=\left[\begin{array}{cc}
0 & 2 \\
0 & -2
\end{array}\right],} \\
{\left[\begin{array}{c}
\partial_{4 k-3} \\
0
\end{array}=\left[\begin{array}{c}
-2 \\
0
\end{array}\right],\right.} & {\left[\partial_{4 k-4}\right]=[0],}
\end{array}
$$

com geradores para $\operatorname{ker}\left(\partial_{i}\right)$ e $\operatorname{im}\left(\partial_{i}\right)$ dados por

\begin{tabular}{c|cccc} 
& $\partial_{4 k-1}$ & $\partial_{4 k-2}$ & $\partial_{4 k-3}$ & $\partial_{4 k-4}$ \\
\hline $\operatorname{ker}$ & 0 & $\tilde{c}_{1}^{4 k-2}+\tilde{c}_{2}^{4 k-2}$ & $\tilde{c}_{2}^{4 k-3}$ & $\tilde{c}^{4 k-4}$ \\
\hline $\operatorname{im}$ & $2\left(\tilde{c}_{1}^{4 k-2}+\tilde{c}_{2}^{4 k-2}\right)$ & $2 \tilde{c}_{2}^{4 k-3}$ & $-2 \tilde{c}^{4 k-4}$ & 0
\end{tabular}

Portanto,

$$
H_{4 k-1}\left(\mathcal{Q} ; \mathbb{Z}_{\rho_{x}}\right)=0, \quad H_{i}\left(\mathcal{Q} ; \mathbb{Z}_{\rho_{x}}\right) \simeq \mathbb{Z}_{2}, \quad i \neq 4 k-1
$$

Seja $\rho_{y}:(x, y) \mapsto(1,-1)$ uma representação não trivial. Aqui podemos ter $t$ par ou ímpar. Em ambos os casos, os grupos de homologia coincidem, ou seja, independem da paridade de $t$. O processo de cálculo é o mesmo, resultando em

$$
H_{4 k-1}\left(\mathcal{Q} ; \mathbb{Z}_{\rho_{y}}\right)=0, \quad H_{4 k-2}\left(\mathcal{Q} ; \mathbb{Z}_{\rho_{y}}\right) \simeq H_{4 k-4}\left(\mathcal{Q} ; \mathbb{Z}_{\rho_{y}}\right) \simeq \mathbb{Z}_{2}, \quad H_{4 k-3}\left(\mathcal{Q} ; \mathbb{Z}_{\rho_{y}}\right)=\mathbb{Z}_{t}
$$

Para a representação $\rho_{x y}:(x, y) \mapsto(-1,-1)$, o resultado é o mesmo que o obtido para $\rho_{x}$. 
Neste capítulo, faremos o cálculo da RS torção das esferas, dos espaços lenticulares, das formas espaciais esféricas quaterniônicas (para estas, também a R torção) e do cone sobre as esferas (em particular, dos discos). Para as três primeiras variedades, o Teorema de CheegerMüller [7, 22] nos fornece a torção analítica destes espaços. Finalizamos com o cálculo da torção analítica do disco, por meio do uso de uma fórmula que a relaciona com a RS torção.

\subsection{Torções das esferas}

Weng e You deram em [30] uma fórmula para a torção analítica de uma esfera com a métrica Euclidiana. Tal resultado foi obtido pelo cálculo direto aplicando a definição da torção analítica (Definição 1.22) e é baseado no conhecimento do espectro do Laplaciano sobre as formas. Provamos nesta seção uma fórmula diferente para a torção analítica de uma esfera e mostramos que esta nova fórmula é equivalente àquela dada em [30]. Nossa prova é baseada em fatos puramente geométricos e topológicos, mais precisamente, no cálculo da RS torção (Definição 1.20) e na aplicação do Teorema de Cheeger-Müller. As principais motivações, apesar de uma diferente prova, são: (1) a abordagem topológica é natural e mais simples; (2) nossa fórmula fornece uma boa interpretação geométrica do resultado.

4.1 TEOREMA (RS TORÇão DA ESFERA): Seja $S_{l}^{n}$ a esfera de raio $l>0$ em $\mathbb{R}^{n+1}(n>0)$ com a métrica Riemanniana $g_{l}$ induzida pelo mergulho. Seja $\rho_{0}$ a representação ortogonal trivial de $\pi_{1}\left(S_{l}^{n}\right)$. Então

$$
\tau_{\mathrm{RS}}\left(\left(S_{l}^{n}, g_{l}\right) ; \rho_{0}\right)=\left\{\begin{array}{cl}
1 & \text { se } n \text { é par } \\
\left(\operatorname{Vol}_{g_{l}}\left(S_{l}^{n}\right)\right)^{\mathrm{rk}\left(\rho_{0}\right)} & \text { se } n \text { é ímpar. }
\end{array}\right.
$$

PROVA: Lembramos que $S_{l}^{n}$ pode ser parametrizada em coordenadas polares por

$$
S_{l}^{n}=\left\{\begin{aligned}
x_{1} & =l \operatorname{sen} \theta_{n} \operatorname{sen} \theta_{n-1} \cdots \operatorname{sen} \theta_{3} \operatorname{sen} \theta_{2} \cos \theta_{1} \\
x_{2} & =l \operatorname{sen} \theta_{n} \operatorname{sen} \theta_{n-1} \cdots \operatorname{sen} \theta_{3} \operatorname{sen} \theta_{2} \operatorname{sen} \theta_{1} \\
& \vdots \\
x_{n} & =l \operatorname{sen} \theta_{n} \cos \theta_{n-1} \\
x_{n+1} & =l \cos \theta_{n}
\end{aligned}\right.
$$


com $0 \leq \theta_{1} \leq 2 \pi, 0 \leq \theta_{2}, \ldots, \theta_{n} \leq \pi$. A métrica Riemanniana induzida é

$$
g_{l}=l^{2}\left(\left(d \theta_{n}\right)^{2}+\left(\operatorname{sen} \theta_{n}\right)^{2}\left(d \theta_{n-1}\right)^{2}+\cdots+\prod_{i=2}^{n}\left(\operatorname{sen} \theta_{i}\right)^{2}\left(d \theta_{1}\right)^{2}\right),
$$

com

$$
\begin{aligned}
\left|g_{l}\right| & =l^{2 n}\left(\operatorname{sen} \theta_{n}\right)^{2(n-1)}\left(\operatorname{sen} \theta_{n-1}\right)^{2(n-2)} \cdots\left(\operatorname{sen} \theta_{3}\right)^{4}\left(\operatorname{sen} \theta_{2}\right)^{2}, \\
\sqrt{\left|g_{l}\right|} & =l^{n}\left(\operatorname{sen} \theta_{n}\right)^{n-1}\left(\operatorname{sen} \theta_{n-1}\right)^{n-2} \cdots\left(\operatorname{sen} \theta_{3}\right)^{2}\left(\operatorname{sen} \theta_{2}\right),
\end{aligned}
$$

isto é, $g_{l}=l^{2} g_{1}$ e $\sqrt{\left|g_{l}\right|}=l^{n} \sqrt{\left|g_{1}\right|}$.

Consideremos a decomposição celular canônica de $S_{l}^{n}$, com uma $n$-célula e uma 0-célula. Seja $\rho_{0}$ a representação trivial quando $n=1$, de 'rank' $m$. Então o complexo relevante é

$$
C: \quad 0 \longrightarrow \mathbb{R}\left\langle c_{n}\right\rangle \longrightarrow 0 \longrightarrow \cdots \longrightarrow 0 \longrightarrow \mathbb{R}\left\langle c_{0}\right\rangle \longrightarrow 0
$$

com bases preferenciais $c_{n}=\left\{c_{1}^{n}\right\}$ e $c_{0}=\left\{c_{1}^{0}\right\}$. Para fixarmos a base para a homologia, precisamos de uma base ortonormal graduada $a$ para as formas harmônicas. Sejam $a_{0}=\left\{a_{1}^{0}\right\}=\left\{\frac{1}{\sqrt{\operatorname{Vol}_{g_{l}}\left(S_{l}^{n}\right)}}\right\}$ e $a_{n}=\left\{a_{1}^{n}\right\}=\left\{\frac{1}{\sqrt{\operatorname{Vol}_{g_{l}}\left(S_{l}^{n}\right)}} \sqrt{\left|g_{l}\right|} d \theta_{1} \wedge \cdots \wedge d \theta_{n}\right\}$. Aplicando a fórmula da equação (1.9) obtemos $h_{0}=\left\{h_{1}^{0}\right\}, h_{n}=\left\{h_{1}^{n}\right\}$, com

$$
\begin{aligned}
& h_{1}^{0}=\mathcal{A}_{0}\left(a_{1}^{0}\right)=\frac{1}{\sqrt{\operatorname{Vol}_{g_{l}}\left(S_{l}^{n}\right)}} \int_{S_{l}^{n}} \sqrt{\left|g_{l}\right|} d \theta_{1} \wedge \cdots \wedge d \theta_{n} c_{1}^{0}=\sqrt{\operatorname{Vol}_{g_{l}}\left(S_{l}^{n}\right)} c_{1}^{0}, \\
& h_{1}^{n}=\mathcal{A}_{n}\left(a_{1}^{n}\right)=\frac{1}{\sqrt{\operatorname{Vol}_{g_{l}}\left(S_{l}^{n}\right)}} \int_{\mathrm{pt}} \star \sqrt{\left|g_{l}\right|} d \theta_{1} \wedge \cdots \wedge d \theta_{n} c_{1}^{n}=\frac{1}{\sqrt{\operatorname{Vol}_{g_{l}}\left(S_{l}^{n}\right)}} c_{1}^{n} .
\end{aligned}
$$

As únicas mudanças de base relevantes são em dimensão 0 e $n$. Como $b_{q}=\emptyset$, para todo $q$, segue que

$$
\left|\operatorname{det}\left(h_{0} / c_{0}\right)\right|=\left(\sqrt{\operatorname{Vol}_{g_{l}}\left(S_{l}^{n}\right)}\right)^{m}, \quad\left|\operatorname{det}\left(h_{n} / c_{n}\right)\right|=\frac{1}{\left(\sqrt{\operatorname{Vol}_{g_{l}}\left(S_{l}^{n}\right)}\right)^{m}} .
$$

Aplicando a definição na equação (1.10), obtemos

$$
\tau_{\mathrm{RS}}\left(\left(S_{l}^{n}, g_{l}\right) ; \rho_{0}\right)=\left(\sqrt{\operatorname{Vol}_{g_{l}}\left(S_{l}^{n}\right)}\right)^{m}\left(\frac{1}{\sqrt{\operatorname{Vol}_{g_{l}}\left(S_{l}^{n}\right)}}\right)^{(-1)^{n} m}
$$

completando a prova do teorema.

4.2 OBSERVAÇÃO: Lembramos que

$$
\Gamma\left(\frac{n}{2}+1\right)=\left\{\begin{array}{cl}
\left(\frac{n}{2}\right) ! & \text { se } n \text { é par, } \\
\sqrt{\pi} \frac{n ! !}{2^{\frac{n+1}{2}}} & \text { se } n \text { é ímpar. }
\end{array}\right.
$$


O volume da esfera $S^{n-1}$ é

$$
\operatorname{Vol}\left(S^{n-1}\right)=\frac{2 \pi^{\frac{n}{2}}}{\Gamma\left(\frac{n}{2}\right)} .
$$

Logo, se queremos as esferas de dimensão ímpar, tomamos $n=2 m$ de modo que

$$
\operatorname{Vol}\left(S^{2 m-1}\right)=\frac{2 \pi^{\frac{2 m}{2}}}{\Gamma\left(\frac{2 m}{2}\right)}=\frac{2 \pi^{m}}{\Gamma(m)}=\frac{2 \pi^{m}}{(m-1) !} .
$$

Portanto, a expressão para a torção analítica calculada por Weng e You [30] coincide com o volume das esferas de dimensão ímpar.

4.3 ОBSERVAÇÃO: Considerando o produto $S_{a}^{n} \times S_{b}^{k}$ das esferas unitárias, tendo 4 células $c_{1}^{0}$, $c_{1}^{n}, c_{1}^{k}$, and $c_{1}^{n+k}$, obtemos

$$
\tau_{\mathrm{RS}}\left(S_{a}^{n} \times S_{b}^{k}\right)=\left\{\begin{array}{cl}
\operatorname{Vol}\left(S_{b}^{k}\right)^{\chi\left(S_{a}^{n}\right)} & n \text { par, } k \text { ímpar } \\
\operatorname{Vol}\left(S_{a}^{n}\right)^{\chi\left(S_{b}^{k}\right)} & k \text { par, } n \text { ímpar } \\
1 & n, k \text { pares ou } n, k \text { ímpares }
\end{array}\right.
$$

em concordância com uma das principais propriedades da torção [17].

\subsection{Torções dos espaços lenticulares}

Os espaços lenticulares são formas espaciais esféricas, obtidas pela ação do grupo cíclico $\mathcal{C}_{n}$ nas esferas. Pela Proposição 2.6, faz sentido considerarmos apenas as esferas de dimensão ímpar. No Apêndice A, apresentamos uma construção mais familiar de tais espaços, com a notação tradicionalmente utilizada. Porém, nesta seção, aplicamos os resultados para o grupo cíclico de modo a calcularmos a RS torção dos espaços lenticulares.

Seja $L=S^{2 s-1} /\left(\hat{\pi}_{q_{1}} \oplus \cdots \oplus \hat{\pi}_{q_{s}}\right)\left(\mathcal{C}_{n}\right)$ o espaço lenticular de dimensão $2 s-1$, como na Observação 2.18. Seja $r_{k}$ o inverso multiplicativo de $q_{k}$ módulo $n$, ou seja, $q_{k} r_{k} \equiv 1 \bmod n$. Sabemos que, ao tomarmos uma representação em $\mathbb{C}$ para $\mathcal{C}_{n}$ que aplica o gerador $x$ em uma raíz primitiva da unidade, o complexo de cadeias

$$
\cdots \longrightarrow \mathbb{Z}\left[\mathcal{C}_{n}\right]\left\langle c^{2 k}\right\rangle \stackrel{L_{x^{r_{k}}}}{\longrightarrow} \mathbb{Z}\left[\mathcal{C}_{n}\right]\left\langle c^{2 k-1}\right\rangle \stackrel{x^{r_{k}-1}}{\longrightarrow} \mathbb{Z}\left[\mathcal{C}_{n}\right]\left\langle c^{2 k-2}\right\rangle \longrightarrow \cdots
$$

torna-se acíclico, já que $\operatorname{mdc}\left(r_{k}, n\right)=1$. Este é o processo utilizado para o cálculo da $\mathrm{R}$ torção dos espaços lenticulares. Para o cálculo da RS torção, usamos uma representação que mantém a homologia, ao menos nas extremidades do complexo. Mais precisamente, seja $\rho_{0}$ a representação trivial em $\mathbb{R}$, aplicando o gerador $x$ em 1. Obtemos então o complexo de cadeias com bases preferenciais $c_{k}=\left\{c_{1}^{k}\right\}$, possuindo um único gerador em cada dimensão,

$$
\cdots \longrightarrow \mathbb{R}\left\langle c_{1}^{2 k+1}\right\rangle \stackrel{0}{\longrightarrow} \mathbb{R}\left\langle c_{1}^{2 k}\right\rangle \stackrel{\times n}{\longrightarrow} \mathbb{R}\left\langle c_{1}^{2 k-1}\right\rangle \longrightarrow \cdots
$$


com ker $\partial_{2 s-1}=\left\langle c_{1}^{2 s-1}\right\rangle$ e ker $\partial_{0}=\left\langle c_{1}^{0}\right\rangle$, resultando na existência de homologia.

Como $\partial_{2 k+1} \equiv 0$ é claro que $b_{2 k}=\emptyset$ e portanto $\tilde{b}_{2 k}=\emptyset$. Em dimensões ímpares, tomamos $b_{2 s-1}=\emptyset$ e $b_{2 k-1}=\left\{n c_{1}^{2 k-1}\right\}$, para $k \neq s$, e portanto $\tilde{b}_{2 s-1}=\emptyset$ e $\tilde{b}_{2 k-1}=\left\{c_{1}^{2 k}\right\}$, para $k \neq s$. Por fim, para a homologia obtemos $h_{0}=\left\{h_{1}^{0}\right\}=\left\{\sqrt{\operatorname{Vol}(L)} c_{1}^{0}\right\}$ e $h_{2 s-1}=\left\{h_{1}^{2 s-1}\right\}=\left\{\frac{1}{\sqrt{\operatorname{Vol}(L)}} c_{1}^{2 s-1}\right\}$.

Fazendo a mudança da base preferencial $c_{k}$ para a base $b_{k} h_{k} \tilde{b}_{k-1}$, obtemos

$$
\begin{aligned}
\left|\operatorname{det}\left(b_{0} h_{0} \tilde{b}_{-1} / c_{0}\right)\right| & =\left|\operatorname{det}\left(h_{0} / c_{0}\right)\right|=\sqrt{\operatorname{Vol}(L)}, \\
\left|\operatorname{det}\left(b_{2 k-1} h_{2 k-1} \tilde{b}_{2 k-2} / c_{2 k-1}\right)\right| & =\left|\operatorname{det}\left(b_{2 k-1} / c_{2 k-1}\right)\right|=n, \\
\left|\operatorname{det}\left(b_{2 k} h_{2 k} \tilde{b}_{2 k-1} / c_{2 k}\right)\right| & =\left|\operatorname{det}\left(\tilde{b}_{2 k-1} / c_{2 k}\right)\right|=1, \\
\left|\operatorname{det}\left(b_{2 s-1} h_{2 s-1} \tilde{b}_{2 s-2} / c_{2 s-1}\right)\right| & =\left|\operatorname{det}\left(h_{2 s-1} / c_{2 s-1}\right)\right|=\frac{1}{\sqrt{\operatorname{Vol}(L)}} .
\end{aligned}
$$

Portanto

$$
\tau_{\mathrm{RS}}\left(L ; \rho_{0}\right)=\frac{1}{n^{s-1}} \sqrt{\operatorname{Vol}(L)}\left(\frac{1}{\sqrt{\operatorname{Vol}(L)}}\right)^{-1}=\frac{1}{n^{s-1}} \operatorname{Vol}(L) .
$$

\subsection{Torções das formas espaciais esféricas quaterniônicas}

Quando definimos as formas espaciais esféricas quaterniônicas, fizemos uso da representação complexa $\alpha: \mathcal{Q}_{4 t} \rightarrow U(2 n, \mathbb{C})$, definida em (3.6). Como $\pi_{1}\left(\mathcal{Q}\left(2 t ; q_{1}, \ldots, q_{n}\right)\right) \simeq \mathcal{Q}_{4 t}$, faremos o cálculo da $\mathrm{R}$ torção utilizando uma representação em $U(2, \mathbb{C})$, obtida pela composição

$$
\rho: \mathcal{Q}_{4 t} \longrightarrow \mathbb{H} \stackrel{A}{\longrightarrow} M(2, \mathbb{C}),
$$

onde $A$ é definida em (1.1).

Mais precisamente, $\operatorname{sejam} \theta=\pi / t$ e $\zeta=\cos \theta+i \operatorname{sen} \theta \in \mathbb{H}_{1}$ raíz $2 t$-ésima da unidade. Definimos então

$$
\rho(x)=A(\zeta)=\left(\begin{array}{cc}
\zeta & 0 \\
0 & \bar{\zeta}
\end{array}\right) \quad \text { e } \quad \rho(y)=A(j)=\left(\begin{array}{cc}
0 & 1 \\
-1 & 0
\end{array}\right) .
$$

Portanto, o complexo de $\mathbb{Z}\left[\mathcal{Q}_{4 t}\right]$-módulos (3.7) passa a ser um complexo de $\mathbb{Z}[\mathbb{H}]$-módulos (ou seja, H-módulos) e além disto deverá ser acíclico. Provemos isto.

4.4 Proposição: O complexo de cadeias de $\mathbb{Z}[\mathbb{H}]$-módulos

$$
\begin{aligned}
\cdots \longrightarrow & \mathbb{Z}[\mathbb{H}]\left\langle\tilde{c}^{4 k-1}\right\rangle \stackrel{\partial_{4 k-1}}{\longrightarrow} \mathbb{Z}[\mathbb{H}]\left\langle\tilde{c}_{1}^{4 k-2}, \tilde{c}_{2}^{4 k-2}\right\rangle \stackrel{\partial_{4 k-2}}{\longrightarrow} \\
\stackrel{\partial_{4 k-2}}{\longrightarrow} & \mathbb{Z}[\mathbb{H}]\left\langle\tilde{c}_{1}^{4 k-3}, \tilde{c}_{2}^{4 k-3}\right\rangle \stackrel{\partial_{4 k-3}}{\longrightarrow} \mathbb{Z}[\mathbb{H}]\left\langle\tilde{c}^{4 k-4}\right\rangle \stackrel{\partial_{4 k-4}}{\longrightarrow} \cdots
\end{aligned}
$$


com operadores bordo dados por

$$
\begin{aligned}
& {\left[\partial_{4 k-1}\right]=\left[\begin{array}{ll}
1-\zeta^{r_{k}} & 1-\zeta^{r_{k}} j
\end{array}\right], \quad\left[\partial_{4 k-2}\right]=\left[\begin{array}{cc}
-N_{\zeta^{r_{k}}} & 1+j \\
1+\zeta^{r_{k}} j & \zeta^{r_{k}}-1
\end{array}\right],} \\
& {\left[\partial_{4 k-3}\right]=\left[\begin{array}{c}
\zeta^{r_{k}}-1 \\
j-1
\end{array}\right], \quad\left[\partial_{4 k-4}\right]=\left[P_{\zeta^{r_{k}, j}}\right],}
\end{aligned}
$$

é acíclico.

Prova: Já temos que o complexo (4.7) é semi-exato, como observado na Seção 3.3.5. Notemos também que $\partial_{4 k-4}=0$, pois $P_{\zeta^{r} k, j}=\left(1+\zeta^{r_{k}}+\cdots+\zeta^{r_{k}(2 t-1)}\right)(1+j)=0$. Concluindo, temos:

1. $\operatorname{ker} \partial_{4 k-1}=\operatorname{im} \partial_{4(k+1)-4}=0$ pois se $\partial_{4 k-1}\left(a \tilde{c}^{4 k-1}\right)=a\left(1-\zeta^{r_{k}}\right) \tilde{c}_{1}^{4 k-2}+a\left(1-\zeta^{r_{k}} j\right) \tilde{c}_{2}^{4 k-2}=0$ então $a\left(1-\zeta^{r_{k}}\right)=0$ e $a\left(1-\zeta^{r_{k}} j\right)=0$. Logo, $a=0$, já que $1-\zeta^{r_{k}}, 1-\zeta^{r_{k}} j$ são não nulos. Também, im $\partial_{4 k-1}=\left\langle\left(1-\zeta^{r_{k}}\right) \tilde{c}_{1}^{4 k-2}+\left(1-\zeta^{r_{k}} j\right) \tilde{c}_{2}^{4 k-2}\right\rangle$.

2. $\operatorname{ker} \partial_{4 k-2}=\operatorname{im} \partial_{4 k-1}$ pois

$$
\begin{aligned}
& \partial_{4 k-2}\left(a \tilde{c}_{1}^{4 k-2}+b \tilde{c}_{2}^{4 k-2}\right)=\left(-a N_{\zeta^{r} k}+b\left(1+\zeta^{r_{k}} j\right)\right) \tilde{c}_{1}^{4 k-3}+\left(a(1+j)+b\left(\zeta^{r_{k}}-1\right)\right) \tilde{c}_{2}^{4 k-3}=0 \\
& \Leftrightarrow\left\{\begin{aligned}
-a N_{\zeta^{r_{k}}}+b\left(1+\zeta^{r_{k}} j\right) & =0 \\
a(1+j)+b\left(\zeta^{r_{k}}-1\right) & =0
\end{aligned}\right. \\
& \Leftrightarrow\left\{\begin{array}{r}
-a\left(1-\zeta^{r_{k}}\right)^{-1}\left(1-\zeta^{r_{k}}\right) N_{\zeta^{r_{k}}}+b\left(1-\zeta^{r_{k}} j\right)^{-1}\left(1-\zeta^{r_{k}} j\right)\left(1+\zeta^{r_{k}} j\right)=0 \\
a\left(1-\zeta^{r_{k}}\right)^{-1}\left(1-\zeta^{r_{k}}\right)(1+j)+b\left(1-\zeta^{r_{k}} j\right)^{-1}\left(1-\zeta^{r_{k}} j\right)\left(\zeta^{r_{k}}-1\right)=0
\end{array}\right. \\
& \Leftrightarrow\left\{\begin{array}{r}
a\left(1-\zeta^{r_{k}}\right)^{-1}\left(\zeta^{r_{k}(t-1)}\right)+b\left(1-\zeta^{r_{k}} j\right)^{-1}\left(1-\left(\zeta^{r_{k}} j\right)^{2}\right)=0 \\
a\left(1-\zeta^{r_{k}}\right)^{-1}\left(1-\zeta^{r_{k}}+j-\zeta^{r_{k}} j\right)+b\left(1-\zeta^{r_{k}} j\right)^{-1}\left(\zeta^{r_{k}}-\zeta^{r_{k}} j \zeta^{r_{k}}-1+\zeta^{r_{k}} j\right)=0
\end{array}\right. \\
& \Leftrightarrow\left\{\begin{aligned}
\left(a\left(1-\zeta^{r_{k}}\right)^{-1}-b\left(1-\zeta^{r_{k}} j\right)^{-1}\right)\left(\zeta^{r_{k}(t-1)}\right) & =0 \\
\left(a\left(1-\zeta^{r_{k}}\right)^{-1}-b\left(1-\zeta^{r_{k}} j\right)^{-1}\right)\left(1-\zeta^{r_{k}}+j-\zeta^{r_{k}} j\right) & =0
\end{aligned}\right. \\
& \Leftrightarrow \quad a\left(1-\zeta^{r_{k}}\right)^{-1}=b\left(1-\zeta^{r_{k}} j\right)^{-1}
\end{aligned}
$$

Também,

$$
\begin{aligned}
& \operatorname{im} \partial_{4 k-2}=\left\langle-N_{\zeta^{r_{k}}} \tilde{c}_{1}^{4 k-3}+(1+j) \tilde{c}_{2}^{4 k-3},\left(1+\zeta^{r_{k}} j\right) \tilde{c}_{1}^{4 k-3}+\left(\zeta^{r_{k}}-1\right) \tilde{c}_{2}^{4 k-3}\right\rangle \\
&=\left\langle-N_{\zeta^{r_{k}}}\left(\zeta^{r_{k}}-1\right)\left(\zeta^{r_{k}}-1\right)^{-1} \tilde{c}_{1}^{4 k-3}+(1+j)(j-1)(j-1)^{-1} \tilde{c}_{2}^{4 k-3},\right. \\
&\left.\left(1+\zeta^{r_{k}} j\right)\left(\zeta^{r_{k}}-1\right)\left(\zeta^{r_{k}}-1\right)^{-1} \tilde{c}_{1}^{4 k-3}+\left(\zeta^{r_{k}}-1\right)(j-1)(j-1)^{-1} \tilde{c}_{2}^{4 k-3}\right\rangle \\
&=\left\langle\left(1-\zeta^{r_{k}}\right)\left[\left(\zeta^{r_{k}}-1\right)^{-1} \tilde{c}_{1}^{4 k-3}-(j-1)^{-1} \tilde{c}_{2}^{4 k-3}\right],\right. \\
&\left.\left(\zeta^{r_{k}}-1+j-\zeta^{r_{k}} j\right)\left[\left(\zeta^{r_{k}}-1\right)^{-1} \tilde{c}_{1}^{4 k-3}-(j-1)^{-1} \tilde{c}_{2}^{4 k-3}\right]\right\rangle \\
&=\left\langle\left(\zeta^{r_{k}}-1\right)^{-1} \tilde{c}_{1}^{4 k-3}-(j-1)^{-1} \tilde{c}_{2}^{4 k-3}\right\rangle .
\end{aligned}
$$


3. $\operatorname{ker} \partial_{4 k-3}=\operatorname{im} \partial_{4 k-2}$ pois

$$
\begin{aligned}
\partial_{4 k-3}\left(a \tilde{c}_{1}^{4 k-3}+b \tilde{c}_{2}^{4 k-3}\right) & =\left(a\left(\zeta^{r_{k}}-1\right)+b(j-1)\right) \tilde{c}^{4 k-4}=0 \\
& \Leftrightarrow a\left(\zeta^{r_{k}}-1\right)+b(j-1)=0 .
\end{aligned}
$$

Claramente, $\operatorname{im} \partial_{4 k-3}=\left\langle\tilde{c}^{4 k-4}\right\rangle$.

4. $\operatorname{ker} \partial_{4 k-4}=\operatorname{im} \partial_{4 k-3}$, já que $\partial_{4 k-4}=0$. Obviamente im $\partial_{4 k-4}=0$.

4.5 OBSERVAÇÃO: O resultado acima ainda é válido se tomarmos uma representação $\rho_{a, b}$ mais geral, ou seja, dados $a, b$ inteiros, $\operatorname{mdc}(a, 2 t)=1$ e $\operatorname{mdc}(b, 4)=1$, seja

$$
\rho_{a, b}(x)=A\left(\zeta^{a}\right)=\left(\begin{array}{cc}
\zeta^{a} & 0 \\
0 & \zeta^{-a}
\end{array}\right) \quad \text { e } \quad \rho_{a, b}(y)=A\left(j^{b}\right)=\left(\begin{array}{cc}
0 & \pm 1 \\
\mp 1 & 0
\end{array}\right) .
$$

No entanto, facilmente vemos que são equivalentes $\rho_{a, 1}$ e $\rho_{a, 3}$ e portanto, podemos omitir o índice $b$ e escrever apenas $\rho_{a}$.

Podemos agora calcular a $\mathrm{R}$ torção $\tau_{\mathrm{R}}\left(\mathcal{Q}\left(2 t ; q_{1}, \ldots, q_{n}\right) ; \rho_{a}\right)$. Antes porém, ressalto a mudança na notação: por simplicidade escreveremos apenas o representante para a classe lateral em $\mathbb{H}^{\times} /\left[\mathbb{H}^{\times}, \mathbb{H}^{\times}\right]$, ou seja, $q\left[\mathbb{H}^{\times}, \mathbb{H}^{\times}\right]$será denotado apenas por $q$.

Escolhemos as bases da seguinte maneira:

$$
\begin{aligned}
b_{4 k-1} & =\emptyset, & b_{4 k-2} & =\left\{\left(1-\zeta^{a r_{k}}\right) \tilde{c}_{1}^{4 k-2}+\left(1-\zeta^{a r_{k}} j\right) \tilde{c}_{2}^{4 k-2}\right\}, \\
b_{4 k-3} & =\left\{-N_{\zeta^{a r_{k}}} \tilde{c}_{1}^{4 k-3}+(1+j) \tilde{c}_{2}^{4 k-3}\right\}, & b_{4 k-4} & =\left\{\tilde{c}^{4 k-4}\right\}, \\
\tilde{b}_{4 k-1} & =\emptyset, & \tilde{b}_{4 k-2} & =\left\{\tilde{c}^{4 k-1}\right\}, \\
\tilde{b}_{4 k-3} & =\left\{\tilde{c}_{1}^{4 k-2}\right\}, & \tilde{b}_{4 k-4} & =\left\{\left(\zeta^{a r_{k}}-1\right)^{-1} \tilde{c}_{1}^{4 k-3}\right\} .
\end{aligned}
$$

Assim, as matrizes relevantes de mudança de base (distintas da matriz identidade) ocorrem nas dimensões $4 k-2$ e $4 k-3$, mais precisamente,

$$
\begin{aligned}
\left(b_{4 k-2} \tilde{b}_{4 k-3} / c_{4 k-2}\right) & =\left(\begin{array}{cc}
1-\zeta^{a r_{k}} & 1-\zeta^{a r_{k}} j \\
1 & 0
\end{array}\right), \\
\left(b_{4 k-3} \tilde{b}_{4 k-4} / c_{4 k-3}\right) & =\left(\begin{array}{cc}
-N_{\zeta^{a r_{k}}} & 1+j \\
\left(\zeta^{a r_{k}}-1\right)^{-1} & 0
\end{array}\right),
\end{aligned}
$$

cujos determinantes não comutativos são, por (1.5) e (1.6),

$$
\begin{aligned}
\operatorname{det}\left(b_{4 k-2} \tilde{b}_{4 k-3} / c_{4 k-2}\right) & =-\left(1-\zeta^{a r_{k}} j\right), \\
\operatorname{det}\left(b_{4 k-3} \tilde{b}_{4 k-4} / c_{4 k-3}\right) & =-\left(-N_{\zeta^{a r_{k}}}\right)\left(\zeta^{a r_{k}}-1\right)^{-1}\left(-N_{\zeta^{a r_{k}}}\right)^{-1}(1+j) \\
& =-\left(\zeta^{a r_{k}}-1\right)^{-1}(1+j) .
\end{aligned}
$$


Então a $\mathrm{R}$ torção é (lembrando que para todo quatérnio não nulo $q^{-1}=\bar{q} /|q|^{2}$ )

$$
\begin{aligned}
\tau_{\mathrm{R}}\left(\mathcal{Q}\left(2 t ; q_{1}, \ldots, q_{n}\right) ; \rho_{a}\right) & =\prod_{k=1}^{n}\left(1-\zeta^{a r_{k}} j\right)(1+j)^{-1}\left(\zeta^{a r_{k}}-1\right) \\
& =\prod_{k=1}^{n} \frac{1}{2}\left(1-\zeta^{a r_{k}} j\right)(1-j)\left(\zeta^{a r_{k}}-1\right),
\end{aligned}
$$

ou seja, é um elemento em $\mathbb{H}^{\times} /\left[\mathbb{H}^{\times}, \mathbb{H}^{\times}\right] \simeq \mathbb{R}^{+}$. Observamos que, para cada $k$, o quatérnio $\left(1-\zeta^{a r_{k}} j\right)(1+j)^{-1}\left(\zeta^{a r_{k}}-1\right)$ possui norma $2(1-\cos \theta)$.

Provamos então o seguinte teorema.

4.6 TEOREMA (R TORÇão): Dado o grupo dos quatérnios generalizado $\mathcal{Q}_{4 t}$, para cada inteiro $a$ primo com $2 t$, seja $\rho_{a}: \mathcal{Q}_{4 t} \rightarrow U(2, \mathbb{C})$ uma representação. Então a torção de Reidemeister da forma espacial esférica quaterniônica, com relação à representação $\rho_{a}$ é

$$
\tau_{\mathrm{R}}\left(\mathcal{Q}\left(2 t ; q_{1}, \ldots, q_{n}\right) ; \rho_{a}\right)=\prod_{k=1}^{n} \frac{1}{2}\left(1-\zeta^{a r_{k}} j\right)(1-j)\left(\zeta^{a r_{k}}-1\right) \in \mathbb{H}^{\times} /\left[\mathbb{H}^{\times}, \mathbb{H}^{\times}\right]
$$

Assim como foi feito para as esferas e os espaços lenticulares, podemos tomar o complexo (3.7), usar a representação trivial para torná-lo um complexo de R-módulos e então calcularmos a RS torção, em função do volume do espaço. Pelo cálculo da homologia feito na página 46, podemos ver que apenas $H_{0}$ e $H_{4 n-1}$ são livres de torção. Após uma escolha natural das bases, temos os determinantes

$$
\operatorname{det}\left(b_{k} \tilde{b}_{k-1} h_{k} / c_{k}\right)= \begin{cases}\sqrt{\operatorname{Vol}(\mathcal{Q})}, & k=0 \\ 1, & k \equiv 1 \bmod 4,0<k<4 n-1 \\ 1 / 4, & k \equiv 2 \bmod 4,0<k<4 n-1 \\ 1, & k \equiv 3 \bmod 4,0<k<4 n-1 \\ 1 / 4 t, & k \equiv 0 \bmod 4,0<k<4 n-1 \\ 1 / \sqrt{\operatorname{Vol}(\mathcal{Q})}, & k=4 n-1\end{cases}
$$

Portanto, temos:

4.7 TEOREMA (RS TORÇÃo): Dado o grupo dos quatérnios generalizado $\mathcal{Q}_{4 t}$, seja $\rho_{0}: \mathcal{Q}_{4 t} \rightarrow \mathbb{R}$ a representação trivial. Então a RS torção da forma espacial esférica quaterniônica, com relação à representação $\rho_{0}$ é

$$
\tau_{\mathrm{RS}}\left(\mathcal{Q}\left(2 t ; q_{1}, \ldots, q_{n}\right) ; \rho_{0}\right)=\frac{1}{4^{n}} \frac{1}{(4 t)^{n-1}} \operatorname{Vol}(\mathcal{Q}) \in \mathbb{R}^{+}
$$




\subsection{RS torção do cone geométrico sobre a esfera}

Nesta seção calculamos a RS torção do disco $m$-dimensional de raio $l>0, D_{l}^{m}$, por meio do caso mais geral de um cone. Mais precisamente, consideraremos o cone $C S^{n}$ construído em $\mathbb{R}^{n+2}$ sobre a esfera $S^{n}, m=n+1$. Claro que $C S^{n}$ não é uma variedade Riemanniana suave, mas é um espaço com singularidade cônica como definida por Cheeger em [8]. Obviamente isto não faz diferença no que diz respeito à parte topológica/algébrica do problema e portanto podemos definir a W torção e a R torção. Quanto à RS torção, precisamos de algum cuidado, desde que não sabemos como se estende a teoria de de Rham, ou seja, nem sempre é possível definir a aplicação de de Rham $\mathcal{A}^{q}$ para espaços com singularidades. No entanto, como veremos a seguir, podemos definir tais aplicações no caso particular de $C S^{n}$ e portanto definimos a RS torção de acordo. Em particular, a construção engloba o caso do disco, enunciado no Corolário 4.10.

Seja $S_{b}^{n}$ a esfera de raio $b>0$ em $\mathbb{R}^{n+1}(n>0), S_{b}^{n}=\left\{x \in \mathbb{R}^{n+1} ;|x|=b\right\}$ (escreveremos apenas $S^{n}$ quando $b=1$ ). Seja $C_{\alpha} S_{l \operatorname{sen} \alpha}^{n}$ o cone de ângulo $\alpha$ sobre $S_{l \operatorname{sen} \alpha}^{n}$ em $\mathbb{R}^{n+2}$. Note que $D_{l}^{n+1}=C_{\frac{\pi}{2}} S_{l}^{n}$. Parametrizamos $C_{\alpha} S_{l \operatorname{sen} \alpha}^{n}$ por

$$
C_{\alpha} S_{l \operatorname{sen} \alpha}^{n}=\left\{\begin{aligned}
x_{1} & =r \operatorname{sen} \alpha \operatorname{sen} \theta_{n} \operatorname{sen} \theta_{n-1} \cdots \operatorname{sen} \theta_{3} \operatorname{sen} \theta_{2} \cos \theta_{1} \\
x_{2} & =r \operatorname{sen} \alpha \operatorname{sen} \theta_{n} \operatorname{sen} \theta_{n-1} \cdots \operatorname{sen} \theta_{3} \operatorname{sen} \theta_{2} \operatorname{sen} \theta_{1} \\
& \vdots \\
x_{n+1}= & r \operatorname{sen} \alpha \cos \theta_{n} \\
x_{n+2} & =r \cos \alpha
\end{aligned}\right.
$$

com $0 \leq r \leq l, 0 \leq \theta_{1} \leq 2 \pi, 0 \leq \theta_{2}, \ldots, \theta_{n} \leq \pi, \alpha$ real positivo fixado e $0<a=\frac{1}{\nu}=\operatorname{sen} \alpha \leq 1$.

A métrica induzida é

$$
\begin{aligned}
g_{E} & =d r \otimes d r+r^{2} a^{2} g_{S^{n}} \\
& =d r \otimes d r+r^{2} a^{2}\left(\sum_{i=1}^{n-1}\left(\prod_{j=i+1}^{n}\left(\operatorname{sen} \theta_{j}\right)\right) d \theta_{i} \otimes d \theta_{i}+d \theta_{n} \otimes d \theta_{n}\right)
\end{aligned}
$$

e $\sqrt{\left|g_{E}\right|}=r^{n} a^{n}\left(\operatorname{sen} \theta_{n}\right)^{n-1}\left(\operatorname{sen} \theta_{n-1}\right)^{n-2} \cdots\left(\operatorname{sen} \theta_{3}\right)^{2}\left(\operatorname{sen} \theta_{2}\right)$.

Seja $K$ a decomposição celular de $C_{\alpha} S_{l \operatorname{sen} \alpha}^{n}$, com uma célula de dimensão máxima, uma $n$ célula e uma 0-célula, $K=c_{1}^{n+1} \cup c_{1}^{n} \cup c_{1}^{0}$. Seja $L$ sub-complexo de $K, L$ sendo a decomposição celular de $S_{l \text { sen } \alpha}^{n}, L=c_{1}^{n} \cup c_{1}^{0}$.

4.8 ProposiçÃo (RS torção (ABSoluta) do cone): Para condições de contorno absolutas temos

$$
\tau_{\mathrm{RS}}\left(\left(C_{\alpha} S_{l \operatorname{sen} \alpha}^{n}, g_{E}\right) ; \rho\right)=\left(\sqrt{\operatorname{Vol}_{g_{E}}\left(C_{\alpha} S_{l \operatorname{sen} \alpha}^{n}\right)}\right)^{\mathrm{rk}(\rho)}
$$


onde $\rho$ é uma representação ortogonal do grupo fundamental.

PROVA: Pela equação (1.11), o complexo relevante é

$$
C_{\mathrm{abs}}: \quad 0 \longrightarrow \mathbb{R}\left\langle c_{1}^{n+1}\right\rangle \longrightarrow \mathbb{R}\left\langle c_{1}^{n}\right\rangle \longrightarrow 0 \longrightarrow \cdots \longrightarrow 0 \longrightarrow \mathbb{R}\left\langle c_{1}^{0}\right\rangle \longrightarrow 0
$$

com bases preferenciais $c_{n+1}=\left\{c_{1}^{n+1}\right\}, c_{n}=\left\{c_{1}^{n}\right\}$ e $c_{0}=\left\{c_{1}^{0}\right\}$. Portanto, $H_{p}(K)=0$, para $p>1$, e $H_{0}(K)=\mathbb{R}\left\langle c_{1}^{0}\right\rangle$. Como a forma constante 1 é uma base para $\Omega^{0}\left(C_{\alpha} S_{l \operatorname{sen} \alpha}^{n}\right)$, temos $a_{0}=\left\{a_{1}^{0}\right\}=\left\{\frac{1}{\sqrt{\operatorname{Vol}_{g_{E}}\left(C_{\alpha} S_{l \operatorname{sen} \alpha}^{n}\right)}}\right\}$. Aplicando a fórmula na equação (1.9) para a aplicação de de Rham, obtemos $h_{0}=\left\{h_{1}^{0}\right\}$, com

$$
\begin{aligned}
h_{1}^{0}=\mathcal{A}_{0}^{\mathrm{abs}}\left(a_{1}^{0}\right) & =\frac{1}{\sqrt{\operatorname{Vol}_{g_{E}}\left(C_{\alpha} S_{l \operatorname{sen} \alpha}^{n}\right)}} \int_{C_{\alpha} S_{l \operatorname{sen} \alpha}^{n}} \star 1 c_{1}^{0} \\
& =\sqrt{\operatorname{Vol}_{g_{E}}\left(C_{\alpha} S_{l \operatorname{sen} \alpha}^{n}\right)} c_{1}^{0} .
\end{aligned}
$$

Como $b_{n}=\left\{c_{1}^{n}\right\}$ e $b_{q}=\emptyset$, para $q \neq n$, então $\tilde{b}_{n}=\left\{c_{1}^{n+1}\right\}$ e $\tilde{b}_{q}=\emptyset$, para $q \neq n$. Assim,

$$
\left|\operatorname{det}\left(\tilde{b}_{n} / c_{n+1}\right)\right|=1, \quad\left|\operatorname{det}\left(b_{n} / c_{n}\right)\right|=1, \quad\left|\operatorname{det}\left(h_{0} / c_{0}\right)\right|=\sqrt{\operatorname{Vol}_{g_{E}}\left(C_{\alpha} S_{l \operatorname{sen} \alpha}^{n}\right)} .
$$

Aplicando a definição na equação (1.10), segue o resultado.

4.9 ProposiçÃo (RS torção (Relativa) do cone): Para condições de contorno relativas te$\operatorname{mos}$

$$
\tau_{\mathrm{RS}}\left(\left(C_{\alpha} S_{l \operatorname{sen} \alpha}^{n}, S_{l \operatorname{sen} \alpha}^{n}, g_{E}\right) ; \rho\right)=\left(\sqrt{\operatorname{Vol}_{g_{E}}\left(C_{\alpha} S_{l \operatorname{sen} \alpha}^{n}\right)}\right)^{(-1)^{n} \operatorname{rk}(\rho)}
$$

onde $\rho$ é uma representação ortogonal do grupo fundamental.

PROVA: O complexo de espaços vetoriais reais em (1.11) é

$$
C_{\mathrm{rel}}: \quad 0 \longrightarrow \mathbb{R}\left\langle c_{1}^{n+1}\right\rangle \longrightarrow 0 \longrightarrow \cdots \longrightarrow 0
$$

com base preferencial $c_{n+1}=\left\{c_{1}^{n+1}\right\}$. Para escolhermos a base para a homologia, precisamos de uma base ortonormal graduada a para as formas harmônicas. Como uma base para $\Omega^{n+1}\left(C_{\alpha} S_{l \operatorname{sen} \alpha}^{n}\right)$ é $\left\{\sqrt{\left|g_{E}\right|} d r \wedge d \theta_{1} \wedge \cdots \wedge d \theta_{n}\right\}$ temos $a_{n+1}=\left\{a_{1}^{n+1}\right\}=\left\{\frac{\sqrt{\left|g_{E}\right|}}{\sqrt{\operatorname{Vol}_{g_{E}}\left(C_{\alpha} S_{l \operatorname{sen} \alpha}^{n}\right)}} d r \wedge d \theta_{1} \wedge \cdots \wedge d \theta_{n}\right\}$. Aplicando a fórmula na equação (1.9) para a aplicação de de Rham, obtemos $h_{n+1}=\left\{h_{1}^{n+1}\right\}$, com

$$
\begin{aligned}
h_{1}^{n+1}=\mathcal{A}_{n+1}^{\mathrm{rel}}\left(a_{1}^{n+1}\right) & =\frac{1}{\sqrt{\operatorname{Vol}_{g_{E}}\left(C_{\alpha} S_{l \text { sen } \alpha}^{n}\right)}} \int_{\mathrm{pt}} \star \sqrt{\left|g_{E}\right|} d r \wedge d \theta_{1} \wedge \cdots \wedge d \theta_{n} c_{1}^{n+1} \\
& =\frac{1}{\sqrt{\operatorname{Vol}_{g_{E}}\left(C_{\alpha} S_{l \text { sen } \alpha}^{n}\right)}} c_{1}^{n+1} .
\end{aligned}
$$


Note que, para todo $q, b_{q}=\emptyset$ e portanto $\tilde{b}_{q}=\emptyset$. Assim, apenas para dimensão $n+1$,

$$
\left|\operatorname{det}\left(h_{n+1} / c_{n+1}\right)\right|=\frac{1}{\sqrt{\operatorname{Vol}_{g_{E}}\left(C_{\alpha} S_{l \operatorname{sen} \alpha}^{n}\right)}} .
$$

Aplicando a definição na equação (1.10), segue o resultado.

4.10 COROLÁRIO (RS torção do disco): A RS torção do disco $D_{l}^{m}$ de raio $l>0$ em $\mathbb{R}^{m} \operatorname{com}$ a métrica Euclidiana $g_{E}$ induzida pelo mergulho no espaço Euclidiano e com uma representação ortogonal $\rho$ do grupo fundamental é

$$
\tau_{\mathrm{RS}}\left(\left(D_{l}^{m}, g_{E}\right) ; \rho\right)=\left(\sqrt{\operatorname{Vol}_{g_{E}}\left(D_{l}^{m}\right)}\right)^{\mathrm{rk}(\rho)} .
$$

Na mesma situação, a RS torção do par $\left(D_{l}^{m}, S_{l}^{m-1}\right)$ é

$$
\tau_{\mathrm{RS}}\left(\left(D_{l}^{m}, S^{m-1}, g_{E}\right) ; \rho\right)=\left(\sqrt{\operatorname{Vol}_{g_{E}}\left(D_{l}^{m}\right)}\right)^{(-1)^{m-1} \operatorname{rk}(\rho)} .
$$

PROVA: Seguem das Proposições 4.8 e 4.9, tomando $\alpha=\frac{\pi}{2}$.

\subsection{Torção analítica do disco}

O objetivo desta seção é calcular (de modo indireto) a torção analítica do disco. Faremos uma breve descrição do processo, apresentando os fatos que nos levam à conclusão do teorema. Algumas provas serão simplificadas e outras omitidas. Para os detalhes, ver [11].

Precisamos de uma fórmula para a razão entre a torção analítica e a torção de Reidemeister. Observamos que, pelo resultado de Cheeger [7], esta razão depende somente de alguns termos geométricos provenientes da geometria da variedade próxima ao bordo. Desde que a singularidade na ponta do cone não afeta a geometria próxima ao bordo, podemos fazer nosso cálculo para o caso geral do cone $C_{\alpha} S_{l \text { sen } \alpha}^{n}$. No entanto, surgiriam algumas dificuldades técnicas que estão além do objetivo deste trabalho. Como observado na Introdução, existem duas fórmulas distintas para tal razão, uma no Teorema 1 de [10] e a outra no Teorema 1 de [5], que será a utilizada aqui.

Basicamente, temos duas etapas para a prova do Teorema 4.13. Primeiro, obtemos do Lema 4.11 uma fórmula para a razão em termos de alguns invariantes geométricos. Isso segue do Teorema 1 de [5] e nos dá, no caso de dimensão ímpar, a razão em termos da característica de Euler do bordo. O caso de dimensão par é bem mais complicado e necessita de algumas notações e ferramentas apresentadas em $[3,5]$. Segundo, o Lema 4.12 fornece o cálculo da integral que aparece na segunda equação do Lema 4.11, concluindo assim o caso de dimensão par.

Considerando a parametrização dada em (4.8) e as métricas

$$
g_{1}=g_{E}=d r \otimes d r+a^{2} r^{2} g_{S^{n}}, \quad g_{0}=d r \otimes d r+a^{2} l^{2} g_{S^{n}},
$$


enunciamos o lema abaixo, onde $\int^{B}$ denota a integral de Berezin e $\mathcal{S}_{1}^{2 p-1}$ é uma forma definida em (1.8) e (1.15) de [5].

4.11 LEMA: A razão entre a torção analítica e a torção de Reidemeister do disco $D_{l}^{m}$, de dimensão ímpar $m=2 p-1$ e de dimensão par $m=2 p(p>0)$, com condições de contorno absolutas são, respectivamente,

$$
\begin{aligned}
\log \frac{T_{\text {abs }}\left(\left(D_{l}^{2 p-1}, g_{1}\right) ; \rho\right)}{\tau_{\mathrm{RS}}\left(\left(D_{l}^{2 p-1}, g_{1}\right) ; \rho\right)}=\frac{1}{4} \operatorname{rk}(\rho) \chi\left(S_{l}^{2 p-2}, g_{E}\right)\left(\log 2+\frac{1}{2} \sum_{n=1}^{p-1} \frac{1}{n}\right), \\
\log \frac{T_{\mathrm{abs}}\left(\left(D_{l}^{2 p}, g_{1}\right) ; \rho\right)}{\tau_{\mathrm{RS}}\left(\left(D_{l}^{2 p}, g_{1}\right) ; \rho\right)}=\frac{1}{2} \operatorname{rk}(\rho) \frac{2^{p-1}}{\sqrt{\pi}(2 p-1) ! !} \sum_{j=1}^{p} \frac{1}{2 j-1} \int_{S_{l}^{2 p-1}} \int^{B} \mathcal{S}_{1}^{2 p-1} .
\end{aligned}
$$

Prova: Temos da equação (0.6) e da Seção 4.1 de [5],

$$
\log \frac{T_{\mathrm{abs}}\left(\left(D_{l}^{m}, g_{1}\right) ; \rho\right)}{T_{\mathrm{abs}}\left(\left(D_{l}^{m}, g_{0}\right) ; \rho\right)}=\frac{1}{2} \operatorname{rk}(\rho) \int_{S_{l}^{m-1}}\left(B\left(\nabla_{1}^{T D_{l}^{m}}\right)-B\left(\nabla_{0}^{T D_{l}^{m}}\right)\right)
$$

onde as formas $B\left(\nabla_{j}^{T X}\right)$ estão definidas na equação (1.17) de [5]. Desde que $g_{0}$ é um produto próximo ao bordo, pelo resultado de [19] temos

$$
\log \frac{T_{\mathrm{abs}}\left(\left(D_{l}^{m}, g_{0}\right) ; \rho\right)}{\tau_{\mathrm{RS}}\left(\left(D_{l}^{m}, g_{1}\right) ; \rho\right)}=\frac{1}{4} \operatorname{rk}(\rho) \chi\left(S^{m-1}, g_{1}\right) \log 2,
$$

ou seja,

$$
\log T_{\mathrm{abs}}\left(\left(D_{l}^{m}, g_{0}\right) ; \rho\right)=\log \tau_{\mathrm{RS}}\left(\left(D_{l}^{m}, g_{1}\right) ; \rho\right)+\frac{1}{4} \operatorname{rk}(\rho) \chi\left(S^{m-1}, g_{1}\right) \log 2 .
$$

Substituindo em (4.11), obtemos

$$
\log \frac{T_{\mathrm{abs}}\left(\left(D_{l}^{m}, g_{1}\right) ; \rho\right)}{\tau_{\mathrm{RS}}\left(\left(D_{l}^{m}, g_{1}\right) ; \rho\right)}=\frac{1}{4} \operatorname{rk}(\rho) \chi\left(S^{m-1}, g_{1}\right) \log 2+\frac{1}{2} \operatorname{rk}(\rho) \int_{S_{l}^{m-1}}\left(B\left(\nabla_{1}^{T D_{l}^{m}}\right)-B\left(\nabla_{0}^{T D_{l}^{m}}\right)\right) .
$$

Resta-nos calcularmos o segundo termo da soma acima.

Se $m=2 p+1(p \geq 0)$ então

$$
\frac{1}{2} \operatorname{rk}(\rho) \int_{S_{l}^{m-1}}\left(B\left(\nabla_{1}^{T D_{l}^{m}}\right)-B\left(\nabla_{0}^{T D_{l}^{m}}\right)\right)=\frac{1}{8} \operatorname{rk}(\rho) \sum_{n=1}^{p} \frac{1}{n} \chi\left(S^{2 p}, g_{1}\right),
$$

provando (4.9).

Se $m=2 p(p \geq 1)$, afirmamos que (detalhes em [11])

$$
\begin{aligned}
B\left(\nabla_{1}^{T D_{l}^{2 p}}\right) & =\frac{1}{2 \Gamma\left(p+\frac{1}{2}\right)} \sum_{j=0}^{p-1} \frac{1}{2 p-2 j-1} \int^{B} \mathcal{S}_{1}^{2 p-1} \\
& =\frac{2^{p}}{2 \sqrt{\pi}(2 p-1) ! !} \sum_{j=0}^{p-1} \frac{1}{2(p-j)-1} \int^{B} \mathcal{S}_{1}^{2 p-1} \\
& =\frac{2^{p-1}}{\sqrt{\pi}(2 p-1) ! !} \sum_{j=1}^{p} \frac{1}{2 j-1} \int^{B} \mathcal{S}_{1}^{2 p-1},
\end{aligned}
$$


e como $B\left(\nabla_{0}^{T D_{l}^{m}}\right)$ se anula (em ambos os casos, na verdade), então

$$
\frac{1}{2} \operatorname{rk}(\rho) \int_{S_{l}^{m-1}}\left(B\left(\nabla_{1}^{T D_{l}^{m}}\right)-B\left(\nabla_{0}^{T D_{l}^{m}}\right)\right)=\frac{1}{2} \operatorname{rk}(\rho) \frac{2^{p-1}}{\sqrt{\pi}(2 p-1) ! !} \sum_{j=1}^{p} \frac{1}{2 j-1} \int_{S_{l}^{m-1}} \int^{B} \mathcal{S}_{1}^{2 p-1},
$$

provando (4.10).

A equação (4.10) do Lema 4.11 pode ser simplificada pelo lema abaixo, cuja prova será omitida por ser extensa e muito técnica, saindo assim dos objetivos em questão, mas que pode ser encontrada em [11, Seção 4, Lema 2].

4.12 LEMA: Na equação (4.12), pode-se mostrar que

$$
\frac{2^{p-1}}{\sqrt{\pi}(2 p-1) ! !} \int_{S_{l}^{2 p-1}} \int^{B} \mathcal{S}_{1}^{2 p-1}=1 .
$$

Com isto, apresentamos:

4.13 TEOREMA (Torção analítica do disco): A torção analítica do disco $D_{l}^{m}$ de raio $l>0$ em $\mathbb{R}^{m}$, com a métrica Euclidiana $g_{E}$ induzida pelo mergulho, com condições de contorno absolutas no bordo e com representação ortogonal $\rho$ para o grupo fundamental é $(p>0)$

$$
\begin{aligned}
\log T_{\mathrm{abs}}\left(\left(D_{l}^{2 p-1}, g_{E}\right) ; \rho\right) & =\frac{1}{2} \operatorname{rk}(\rho) \log \operatorname{Vol}_{g_{E}}\left(D_{l}^{2 p-1}\right)+\frac{1}{2} \operatorname{rk}(\rho) \log 2+\frac{1}{4} \operatorname{rk}(\rho) \sum_{n=1}^{p-1} \frac{1}{n} \\
\log T_{\mathrm{abs}}\left(\left(D_{l}^{2 p}, g_{E}\right) ; \rho\right) & =\frac{1}{2} \operatorname{rk}(\rho) \log \operatorname{Vol}_{g_{E}}\left(D_{l}^{2 p}\right)+\frac{1}{2} \operatorname{rk}(\rho) \sum_{n=1}^{p} \frac{1}{2 n-1}
\end{aligned}
$$

Prova: Pela Proposição 4.8, temos

$$
\log \tau_{\mathrm{RS}}\left(\left(D_{l}^{m}, g_{E}\right) ; \rho\right)=\frac{1}{2} \mathrm{rk}(\rho) \log \operatorname{Vol}_{g_{E}}\left(D_{l}^{m}\right) .
$$

Como a característica de Euler de uma esfera de dimensão par é 2, por (4.9) temos a prova para $m$ ímpar. O caso $m$ par segue por simples substituição da igualdade acima em (4.10), juntamente com o Lema 4.12. 


\section{APÊNDICE A}

\section{ESPAÇOS LENTICULARES}

Ilustramos neste Apêndice o uso da $\mathrm{R}$ torção na classificação de espaços. Definições e resultados são apresentados, como por exemplo, a estrutura celular e o cálculo da homologia de tais espaços, e por fim faz-se a classificação por homotopia e homotopia simples. O leitor perceberá grande semelhança com a construção realizada no Capítulo 3, bem como certa familiaridade com a notação aqui utilizada. Para maiores detalhes, consultar [9].

Seja $p \geq 2$ um inteiro (não necessariamente primo) e sejam $q_{1}, q_{2}, \ldots, q_{n}$ inteiros relativamente primos com $p$, isto é, $\operatorname{mdc}\left(q_{j}, p\right)=1$. Então, o espaço lenticular $L\left(p ; q_{1}, \ldots, q_{n}\right)$ é uma variedade $(2 n-1)$-dimensional, a qual definiremos como $\Sigma^{2 n-1} / G$, para apropriados $\Sigma^{2 n-1}$ e $G$.

Se $p>2$, seja $\Sigma^{1}$ o polígono regular (1-esfera simplicial) em $\mathbb{R}^{2}$ com vértices $v_{q}=e^{2 \pi i q / p}$, $q=0,1,2, \ldots, p-1$. Seja $\Sigma^{2 n-1}$ o poliedro em $\mathbb{R}^{2 n}=\mathbb{R}^{2} \times \cdots \times \mathbb{R}^{2}$ obtido fazendo o 'join' iterado

$$
\Sigma^{2 n-1}=\Sigma_{1} * \Sigma_{2} * \cdots * \Sigma_{n}=\left\{\lambda_{1} z_{1}+\cdots+\lambda_{n} z_{n} ; \sum_{j} \lambda_{j}=1, \lambda_{j} \geq 0, z_{j} \in \Sigma_{j}\right\}
$$

onde $\Sigma_{j}$ é a cópia de $\Sigma^{1}$ na $j$-ésima cópia de $\mathbb{R}^{2}$ em $\mathbb{R}^{2 n}$ e cada $z \in \Sigma^{2 n-1}$ é unicamente expresso como uma tal soma. $\Sigma^{2 n-1}$ é um complexo simplicial e, como 'join' de circunferências, é uma $(2 n-1)$-esfera.

Quando $p=2$, devemos alterar o procedimento acima (já que dois pontos não determinam uma circunferência). Seja $\Sigma^{1}$ o polígono regular com vértices $v_{0}=1, A=e^{\pi i / 2}, v_{1}=e^{\pi i}$ e $B=e^{3 \pi i / 2}$. $\Sigma^{2 n-1}$ é então descrita como acima.

Para construirmos um grupo $G$ que atue em $\Sigma^{2 n-1}$, seja $R_{j}$ a rotação de $\Sigma_{j}$ por $q_{j} \theta$, onde $\theta=2 \pi / p$ radianos. Seja $g=R_{1} * R_{2} * \cdots * R_{n}: \Sigma^{2 n-1} \rightarrow \Sigma^{2 n-1}$, isto é,

$$
g\left(\sum_{j} \lambda_{j} z_{j}\right)=\sum_{j} \lambda_{j} R_{j}\left(z_{j}\right)
$$

Sendo um 'join' de isomorfismos simpliciais, $g$ é um isomorfismo simplicial. Claramente $g^{p}=1$, mas se $1 \leq k \leq p-1, g^{k}$ não fixa pontos de $\Sigma^{2 n-1}$, pois se $z=\sum_{j=1}^{n} \lambda_{j} z_{j}$, com $\lambda_{j_{0}} \neq 0$, então

$$
\operatorname{mdc}\left(q_{j_{0}}, p\right)=1 \Rightarrow R_{j_{0}}^{k}\left(z_{j_{0}}\right) \neq z_{j_{0}} \Rightarrow g^{k}(z)=\sum_{j} \lambda_{j} R_{j}^{k}\left(z_{j}\right) \neq \sum_{j} \lambda_{j} z_{j}=z
$$

Portanto, $G=\left\{1, g, g^{2}, \ldots, g^{p-1}\right\}$ é um grupo de ordem $p$ de homeomorfismos que atua livremente em $\Sigma^{2 n-1}$ e com isto definimos

$$
L\left(p ; q_{1}, \ldots, q_{n}\right)=\Sigma^{2 n-1} / G .
$$


Claramente, $L=L\left(p ; q_{1}, \ldots, q_{n}\right)$ é uma variedade e $\pi: \Sigma^{2 n-1} \rightarrow L$ é uma cobertura com $G$ como grupo das transformações cobertura.

A.1 ObservaçÃo: $L\left(p ; q_{1}, \ldots, q_{n}\right)$ pode também naturalmente ser definido como uma variedade diferenciável tratando-o como um quociente da esfera $S^{2 n-1}$. Seja $\bar{g}: \mathbb{R}^{2 n} \rightarrow \mathbb{R}^{2 n}$ dada por $\bar{g}\left(z_{1}, \ldots, z_{n}\right)=\left(R_{1}\left(z_{1}\right), \ldots, R_{n}\left(z_{n}\right)\right)$, onde $R_{j}$ é a rotação de $\mathbb{R}^{2}$ por $2 \pi q_{j} / p$ radianos. Então $\bar{g}$ é uma transformação ortogonal tal que $\bar{g}, \bar{g}^{2}, \ldots, \bar{g}^{p-1}$ não têm pontos fixos além de 0 . Portanto $\bar{G}=\left\{\bar{g}_{\mid S^{2 n-1}}^{k} ; 0 \leq k \leq p-1\right\}$ é um grupo de difeomorfismos de $S^{2 n-1}$ e $S^{2 n-1} / \bar{G}$ é uma variedade diferenciável.

A conexão com $\Sigma^{2 n-1} / G$ é feita notando que $g=\bar{g}_{\mid \Sigma^{2 n-1}}$ e que, se $T: \Sigma^{2 n-1} \rightarrow S^{2 n-1}$ é dada por $T(z)=z /|z|$, o seguinte diagrama comuta.

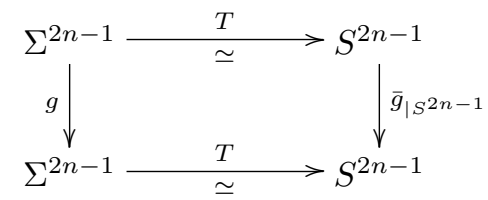

\section{A.1 O espaço tridimensional $L_{p, q}$}

Seja $D^{3}$ a bola unitária fechada em $\mathbb{R}^{3}$ e sejam $D_{+}^{2}$ e $D_{-}^{2}$ os hemisférios fechados superiores e inferiores do bordo $\partial D^{3}$. Sejam $p, q$ inteiros $\operatorname{com} p \geq 2$ e $\operatorname{mdc}(p, q)=1$. Seja $R$ a rotação de $\mathbb{R}^{2}$ por $2 \pi q / p$ radianos e definimos $h: D_{-}^{2} \rightarrow D_{+}^{2}$ por $h(x, y, z)=(R(x, y),-z)$. Deste modo, o espaço lenticular tridimensional $L_{p, q}$ é geralmente definido como o espaço quociente pela relação de equivalência gerada por $h$.

Tentaremos intuir porque $L_{p, q}$ é, a menos de homeomorfismo, o espaço que chamamos de $L(p ; q, 1)$ na seção anterior.

Considere a esfera $S^{3}$ como a compactificação de $\mathbb{R}^{3}$, isto é, $S^{3}=\mathbb{R}^{3} \cup\{\infty\}$. Sejam $\Sigma_{1}$ a circunferência unitária em $\mathbb{R}^{2} \times\{0\}$ e $\Sigma_{2}=($ eixo- $z) \cup\{\infty\}$. Então $S^{3}$ pode ser vista como $\Sigma_{1} * \Sigma_{2}$, como união de uma família de "cones curvados" $v * \Sigma_{1}$, para $v \in \Sigma_{2}$. Por exemplo,

$$
\begin{array}{lll}
v=(0,0,0) & \Rightarrow & v * \Sigma_{1}=B^{2} \times\{0\}, \\
v=(0,0,1) & \Rightarrow & v * \Sigma_{1}=D_{+}^{2}, \\
v=(0,0,-1) & \Rightarrow & v * \Sigma_{1}=D_{-}^{2}, \\
v=\infty & \Rightarrow & v * \Sigma_{1}=\left\{(x, y, 0) ; x^{2}+y^{2} \geq 1\right\} .
\end{array}
$$

Cada um destes "cones" é obtido rotacionando um arco de $v$ a $(0,1,0)$, que está no plano-yz, ao redor do eixo- $z$. 
Seja $R_{1}: \Sigma_{1} \rightarrow \Sigma_{1}$ a rotação de $2 \pi q / p$ radianos. Decompomos $\Sigma_{2}$ em $p$ segmentos, um deles sendo o segmento finito de $(0,0,-1)$ a $(0,0,1)$ e um outro sendo o segmento infinito que tem $\infty$ como ponto interior. Seja $R_{2}: \Sigma_{2} \rightarrow \Sigma_{2}$ o isomorfismo simplicial que levanta cada vértice ao próximo acima, exceto pelo mais alto vértice que se tornará o mais baixo. Desde que todo ponto de $S^{3}-\left(\Sigma_{1} \cup \Sigma_{2}\right)$ está em um único arco de $\Sigma_{1}$ a $\Sigma_{2}$, podemos definir $g=R_{1} * R_{2}$ : $S^{3} \rightarrow S^{3}$ por $g\left[z_{1}, z_{2}, t\right]=\left[R_{1}\left(z_{1}\right), R_{2}\left(z_{2}\right), t\right]$, onde $[a, b, t]$ denota o ponto que está a $t \ell_{a b}$ unidades de comprimento ao longo do arco de $a$ a $b, \ell_{a b}$ sendo o comprimento deste arco. Se tomarmos $G=\left\{1, g, g^{2}, \ldots, g^{p-1}\right\}$ então $S^{3} / G \simeq L(p ; q, 1)$ como definido na seção anterior. Por outro lado, se $\pi: S^{3} \rightarrow S^{3} / G$ é a aplicação quociente, segue de $\pi g^{k}=\pi$ e $S^{3}=\cup_{k} g^{k}\left(B^{3}\right)$ que $\pi\left(B^{3}\right)=S^{3} / G$. Assim $S^{3} / G$ é homeomorfo ao espaço quociente de $D^{3}$ pelas identificações induzidas por $\pi_{\mid D^{3}}$. Mas tal espaço quociente é precisamente $D^{3} / h$ desde que $g_{\mid D_{-}^{2}}=h_{\mid D_{-}^{2}}, g\left(D^{3}\right) \cap D^{3}=D_{+}^{2}$ e $g^{k}\left(D^{3}\right) \cap D^{3}=\emptyset$ se $k \not \equiv \pm 1 \bmod p$. Portanto

$$
L(p ; q, 1) \simeq S^{3} / G \simeq D^{3} / h=L_{p, q} .
$$

\section{A.2 Estrutura celular e grupos de homologia}

Quando $p>2$ denotamos os vértices de $\Sigma^{1}$ por $v_{j}=e^{2 \pi i j / p}$ e os 1-simplexos por $I_{j}=\left[v_{j}, v_{j+1}\right]$, $0 \leq j \leq p-1$. Quando $p=2$ os vértices são $v_{0}, A, v_{1}, B$ e definimos $I_{0}=\left[v_{0}, A\right] \cup\left[A, v_{1}\right] \mathrm{e}$ $I_{1}=\left[v_{1}, B\right] \cup\left[B, v_{0}\right]$, para $A, B$ como anteriormente. Para $0 \leq i \leq n-1$, os seguintes subcomplexos simpliciais de $\Sigma^{2 n-1}$ são importantes (onde $\Sigma^{-1}=\emptyset, E_{j}^{0}=v_{j}$ e $E_{j}^{1}=I_{j}$ ):

$$
\begin{aligned}
\Sigma^{2 i-1} & =\Sigma_{1} * \Sigma_{2} * \cdots * \Sigma_{i} \subset \mathbb{R}^{2 i}, \\
E_{j}^{2 i} & =\Sigma^{2 i-1} * v_{j} \quad\left(v_{j} \in \Sigma_{i+1}\right), \\
E_{j}^{2 i+1} & =\Sigma^{2 i-1} * I_{j}<\Sigma^{2 i+1} .
\end{aligned}
$$

Por exemplo, $D^{3}, D_{-}^{2}, D_{+}^{2}$ correspondem a $E_{j}^{3}, E_{j}^{2}, E_{j+1}^{2}$ onde $v_{j}=(0,0,-1)$ e $v_{j+1}=(0,0,1)$.

Os $E_{j}^{k}$ são bolas fechadas que fornecem uma decomposição CW para $\Sigma^{2 n-1}$ cujas células são $e_{j}^{k}=\operatorname{Int}\left(E_{j}^{k}\right)(0 \leq j \leq p-1,0 \leq k \leq 2 n-1)$. Segue, de propriedades elementares do 'join', que

$$
\begin{aligned}
& \partial E_{j}^{2 i}=\Sigma^{2 i-1}, \quad \partial E_{j}^{2 i+1}=E_{j}^{2 i} \cup E_{j+1}^{2 i}, \quad E_{j}^{2 i} \cap E_{k}^{2 i}=\Sigma^{2 i-1} \quad(j \neq k), \\
& E_{j}^{2 i+1} \cap E_{k}^{2 i+1}= \begin{cases}\Sigma^{2 i-1}, & j-k \neq \equiv 0, \pm 1 \bmod p \\
E_{j+1}^{2 i}, & k \equiv j+1 \bmod p, p \neq 2 \\
E_{0}^{2 i} \cup E_{1}^{2 i}, & j=0, k=1, p=2 .\end{cases}
\end{aligned}
$$


Orientamos $E_{j}^{k}$ (tratando-os por um momento como cadeias simpliciais) estipulando que $E_{j}^{0}=v_{j}$ é positivamente orientado e, indutivamente, que $E_{j}^{2 i+1}$ é orientado $(i \geq 0)$ tal que $\partial E_{j}^{2 i+1}=$ $E_{j+1}^{2 i}-E_{j}^{2 i}, \Sigma^{2 i+1}$ é orientado tal que $E_{j}^{2 i+1} \hookrightarrow \Sigma^{2 i+1}$ preserva orientação e $E_{j}^{2 i+2}$ é orientado tal que $\partial E_{j}^{2 i+2}=\Sigma^{2 i+1}=E_{0}^{2 i+1}+E_{1}^{2 i+1}+\cdots+E_{p-1}^{2 i+1}$. As orientações de $E_{j}^{k}$ naturalmente determinam elementos base para o complexo de cadeias celular $C_{k}\left(\Sigma^{2 n-1}\right)$ determinado por esta estrutura CW e usaremos $e_{j}^{k}$ para denotarmos tais elementos.

Considerando $\Sigma^{2 n-1}$ como a cobertura universal de $L\left(p ; q_{1}, \ldots, q_{n}\right)=\Sigma^{2 n-1} / G$, torna-se natural (como veremos abaixo) denotarmos $e_{0}^{k}=\tilde{e}^{k}(0 \leq k \leq 2 n-1)$. Se $g=R_{1} * R_{2} * \cdots * R_{n}$ como antes, notemos que $g_{\mid \Sigma^{2 i-1}}^{t}: \Sigma^{2 i-1} \rightarrow \Sigma^{2 i-1}$ é um isomorfismo simplicial que preserva orientação (desde que é homotópico à identidade) de modo que $g_{\mid E_{0}^{2 i}}^{t}=\left(g_{\mid \Sigma^{2 i-1}}^{t}\right) *\left(g_{\mid v_{0}}^{t}\right)$ e $g_{\mid E_{0}^{2 i+1}}^{t}=\left(g_{\mid \Sigma^{2 i-1}}^{t}\right) *\left(g_{\mid I_{0}}^{t}\right)$. Assim, $g^{t}$ aplica células orientadas isomorficamente em células orientadas, preservando orientação, e as cadeias celulares básicas satisfazem

$$
\begin{array}{rlr}
e_{j}^{k} & =g^{t} \tilde{e}^{k}, & t q^{j} \equiv j \bmod p \\
\partial \tilde{e}^{2 i+1} & =g^{r_{i+1}} \tilde{e}^{2 i}-\tilde{e}^{2 i}, & r_{i+1} q_{i+1} \equiv 1 \bmod p \\
\partial \tilde{e}^{2 i} & =\tilde{e}^{2 i-1}+g \tilde{e}^{2 i-1}+\cdots+g^{p-1} \tilde{e}^{2 i-1} & \\
\partial g & =g \partial: C_{k}\left(\Sigma^{2 n-1}\right) \rightarrow C_{k}\left(\Sigma^{2 n-1}\right) &
\end{array}
$$

$L\left(p ; q_{1}, \ldots, q_{n}\right)$ obtém uma estrutura CW natural, da estrutura celular de $\Sigma^{2 n-1}$, via projeção $\pi: \Sigma^{2 n-1} \rightarrow L\left(p ; q_{1}, \ldots, q_{n}\right)$, com exatamente uma célula em cada dimensão. As células são

$$
e^{k}=\pi\left(\tilde{e}^{k}\right)=\pi\left(e_{j}^{k}\right), \quad(0 \leq j \leq p-1,0 \leq k \leq 2 n-1),
$$

com aplicações características $\pi_{\mid E_{0}^{k}}: E_{0}^{k} \rightarrow L\left(p ; q_{1}, \ldots, q_{n}\right)$. A orientação que escolhemos para $\tilde{e}^{k}$ induz uma orientação para $e^{k}$. Mais precisamente, a aplicação cadeia induzida pela aplicação celular $\pi$ aplica um elemento básico $\tilde{e}^{k}$ de $C_{k}\left(\Sigma^{2 n-1}\right)$ em um elemento básico $e^{k}$ de $C_{k}\left(L\left(p ; q_{1}, \ldots, q_{n}\right)\right)$.

Para calcularmos $H_{*}\left(L\left(p ; q_{1}, \ldots, q_{n}\right)\right)$, notemos que

$$
\begin{aligned}
\partial e^{2 i} & =\partial \pi\left(\tilde{e}^{2 i}\right)=\pi \partial\left(\tilde{e}^{2 i}\right) \\
& =\pi\left(\tilde{e}^{2 i-1}+g \tilde{e}^{2 i-1}+\cdots+g^{p-1} \tilde{e}^{2 i-1}\right)=p e^{2 i-1}, \\
\partial e^{2 i+1} & =\pi \partial\left(\tilde{e}^{2 i+1}\right)=\pi\left(g^{r_{i+1}} \tilde{e}^{2 i}-\tilde{e}^{2 i}\right)=0 .
\end{aligned}
$$

Assim, o complexo celular é

$$
0 \longrightarrow C_{2 n-1} \stackrel{0}{\longrightarrow} C_{2 n-2} \stackrel{\times p}{\longrightarrow} C_{2 n-3} \stackrel{0}{\longrightarrow} \cdots \stackrel{\times p}{\longrightarrow} C_{1} \stackrel{0}{\longrightarrow} C_{0} \longrightarrow 0 .
$$

Portanto, os grupos de homologia de $L\left(p ; q_{1}, \ldots, q_{n}\right)$ com coeficientes inteiros são

$$
H_{2 n-1} \simeq \mathbb{Z}, \quad H_{2 i-1} \simeq \mathbb{Z}_{p} \quad(1 \leq i<n), \quad H_{2 i}=0 \quad(i>0), \quad H_{0} \simeq \mathbb{Z} .
$$


Desde que a esfera é a cobertura universal dos espaços lenticulares e $G$ é o grupo das transformações cobertura, $\pi_{1}\left(L\left(p ; q_{1}, \ldots, q_{n}\right)\right) \simeq \mathbb{Z}_{p}$ e $\pi_{i}\left(L\left(p ; q_{1}, \ldots, q_{n}\right)\right) \simeq \pi_{i}\left(S^{2 n-1}\right)$, para $i \neq 1$.

A.2 OBservação: A discussão acima mostra que os diferentes $(2 n-1)$-espaços lenticulares determinados por um $p$ fixado têm os mesmos grupos de homologia e homotopia. No entanto, mostraremos rapidamente que nem todos têm o mesmo tipo de homotopia.

\section{A.3 Classificação homotópica}

Colocaremos aqui os principais resultados que nos darão a classificação homotópica dos espaços lenticulares, ilustrando assim a Observação A.2. Omitiremos as provas, por serem extensas e não tão ilustrativas e relevantes quanto os resultados a que se referem. Para maiores detalhes, ver [9, $\S 29]$.

Suponhamos $p \geq 2$ e $\left(q_{1}, \ldots, q_{n}\right),\left(q_{1}^{\prime}, \ldots, q_{n}^{\prime}\right) n$-uplas de inteiros relativamente primos com $p$. Sejam $R_{j}$ e $R_{j}^{\prime}$ rotações de $\Sigma_{j}$ de $q_{j} \theta$ e $q_{j}^{\prime} \theta, \theta=2 \pi / p$, respectivamente. Sejam $g=R_{1} * \cdots * R_{n}$, $g^{\prime}=R_{1}^{\prime} * \cdots * R_{n}^{\prime}$ e sejam $G, G^{\prime}$ os grupos cíclicos (de ordem $p$ ) gerados por $g, g^{\prime}$. Denotemos por $L=L\left(p ; q_{1}, \ldots, q_{n}\right)=\Sigma^{2 n-1} / G$ e $L^{\prime}=L\left(p ; q_{1}^{\prime}, \ldots, q_{n}^{\prime}\right)=\Sigma^{2 n-1} / G^{\prime}$, com aplicações quociente $\pi$ e $\pi^{\prime}$. Sejam também $e^{k}=\pi\left(\tilde{e}^{k}\right)$ e $e^{\prime k}=\pi^{\prime}\left(\tilde{e}^{k}\right)$, onde $\tilde{e}^{k}$ foi definida na página 64 .

A.3 DefiniçÃo: Se $f: \Sigma^{2 n-1} \rightarrow \Sigma^{2 n-1}$ é uma aplicação e se $g \in G, h \in G^{\prime}$ então $f$ é $(g, h)$-equivariante se, e somente se, $f g=h f$. Duas aplicações $f_{0}$ e $f_{1}(g, h)$-equivariantes são equivariantemente homotópicas se existe uma homotopia $f_{t}: \Sigma^{2 n-1} \rightarrow \Sigma^{2 n-1}$ tal que $f_{t}$ é $(g, h)$ equivariante, para todo $t$.

Seguem alguns resultados sobre aplicações $(g, h)$-equivariantes de $\Sigma^{2 n-1}$.

A.4 LEMA: Se $f_{0}$ e $f_{1}$ são aplicações $(g, h)$-equivariantes de $\Sigma^{2 n-1} \operatorname{então} \operatorname{deg}\left(f_{0}\right) \equiv \operatorname{deg}\left(f_{1}\right) \bmod p$. Se $\operatorname{deg}\left(f_{0}\right)=\operatorname{deg}\left(f_{1}\right)$ então $f_{0}$ e $f_{1}$ são equivariantemente homotópicas.

A questão de quais classes de resíduos $\bmod p$ são determinadas pelo grau de uma aplicação equivariante e quais números na classe de resíduos podem ser realizados, é respondida por:

A.5 Proposição: Se $d, a \in \mathbb{Z}$ então existe uma aplicação $\left(g, g^{\prime a}\right)$-equivariante $f: \Sigma^{2 n-1} \rightarrow$ $\Sigma^{2 n-1}$ de grau $d$ se, e somente se, $d \equiv a^{n} r_{1} \cdots r_{n} q_{1}^{\prime} \cdots q_{n}^{\prime} \bmod p, \operatorname{com} r_{j} q_{j} \equiv 1 \bmod p$.

Podemos considerar os resultados acima para aplicações entre espaços lenticulares. 
A.6 Proposição: Suponhamos que $L=L\left(p ; q_{1}, \ldots, q_{n}\right)$ e $L^{\prime}=L\left(p ; q_{1}^{\prime}, \ldots, q_{n}^{\prime}\right)$ são orientados pela escolha de $e^{2 n-1}$ e $e^{2 n-1}$ como geradores de $H_{2 n-1}$. Então:

1. Dadas $f_{0}, f_{1}: L \rightarrow L^{\prime}$

$$
f_{0} \sim f_{1} \Leftrightarrow \operatorname{deg}\left(f_{0}\right)=\operatorname{deg}\left(f_{1}\right), f_{0 \#}=f_{1 \#}: G \rightarrow G^{\prime} .
$$

2. Sejam $d, a \in \mathbb{Z}$. Existe uma aplicação $f: L \rightarrow L^{\prime} \operatorname{com} \operatorname{deg}(f)=d$ e $f_{\#}(g)=g^{a}$ se, e somente se, $d \equiv a^{n} r_{1} \cdots r_{n} q_{1}^{\prime} \cdots q_{n}^{\prime} \bmod p, \operatorname{com} r_{j} q_{j} \equiv 1 \bmod p$.

3. Dada $f: L \rightarrow L^{\prime}, f$ é equivalência de homotopia se, e somente se, $\operatorname{deg}(f)= \pm 1$.

Note que, quando $p=2, L=L^{\prime}=L(2 ; 1, \ldots, 1)=\mathbb{R} P^{2 n-1}$ é o espaço projetivo real. A proposição anterior nos diz que existem exatamente duas auto-equivalências de homotopia de $\mathbb{R} P^{2 n-1}$, uma de grau 1 e outra de grau -1 . Todos os outros casos são dados pela seguinte classificação de equivalências de homotopia.

A.7 TeOremA: Sejam $L=L\left(p ; q_{1}, \ldots, q_{n}\right)$ e $L^{\prime}=L\left(p ; q_{1}^{\prime}, \ldots, q_{n}^{\prime}\right)$, com $p>2, r_{j} q_{j} \equiv 1 \bmod p$ e $r_{j}^{\prime} q_{j}^{\prime} \equiv 1 \bmod p$, para todo $j$. Denotemos por $\mathcal{E}\left[L, L^{\prime}\right]$ o conjunto das classes de equivalência por homotopia de equivalências de homotopia $f: L \rightarrow L^{\prime}$. Então existe uma bijeção

$$
\phi: \mathcal{E}\left[L, L^{\prime}\right] \rightarrow\left\{a ; 0<a<p, a^{n} \equiv \pm q_{1} \cdots q_{n} r_{1}^{\prime} \cdots r_{n}^{\prime} \bmod p\right\}
$$

dada por $\phi([f])=a$ se $f_{\#}(g)=g^{\prime a}$. Além disto, se $\phi([f])=a$ então $\operatorname{deg}(f)= \pm 1$, onde o sinal coincide com o acima.

A.8 Corolário: $L_{p, q}$ e $L_{p, q^{\prime}}$ têm o mesmo tipo de homotopia se, e somente se, existe um inteiro $b$ tal que $q q^{\prime} \equiv \pm b^{2} \bmod p$. Assim, temos os exemplos

$$
L_{5,1} \nsim L_{5,2} \quad \text { e } \quad L_{7,1} \sim L_{7,2},
$$

onde " " denota equivalência de homotopia. No entanto, $L_{7,1}$ e $L_{7,2}$ não têm o mesmo tipo de homotopia simples devido ao Teorema A.9 abaixo. De fato, se $f: L_{7,1} \rightarrow L_{7,2}$ é uma equivalência de homotopia tal que $f_{\#}(g)=g^{\prime a} \operatorname{com} a^{2} \equiv 2 \bmod 7$ ou $a^{2} \equiv 5 \bmod 7$, e se $\zeta=e^{2 \pi i / 7}$, então

$$
\left|(\zeta-1)^{2}\right| \neq\left|\left(\zeta^{a}-1\right)\left(\zeta^{4 a}-1\right)\right|
$$

A.9 TeOremA: Sejam $L=L\left(p ; q_{1}, \ldots, q_{n}\right)$ e $L^{\prime}=L\left(p ; q_{1}^{\prime}, \ldots, q_{n}^{\prime}\right)$ e suponha que $f: L \rightarrow L^{\prime}$ é uma equivalência de homotopia simples. Se $f_{\#}(g)=g^{\prime a}$ então existem números $\epsilon_{i}= \pm 1$ tais que $\left(q_{1}, \ldots, q_{n}\right)$ é igual (módulo $\left.p\right)$ a alguma permutação de $\left(\epsilon_{1} a q_{1}^{\prime}, \ldots, \epsilon_{n} a q_{n}^{\prime}\right)$. 


\section{GEOMETRIA}

Finalizamos este trabalho, apresentando ao leitor os conceitos básicos de Geometria utilizados anteriormente, de modo que o leitor possa consultar este Apêndice com facilidade. Devido à grande quantidade de definições e resultados, omitimos a maioria das provas, que podem ser encontradas na referência [24].

\section{B.1 Tensores e formas diferenciais}

Nota: Desde o início, alertamos o leitor para o uso da "soma de Einstein": se o mesmo índice aparece duas vezes, sobrescrito e subscrito, então o índice é somado sobre todos os valores possíveis. Por exemplo, se $\mu$ varia de 1 a $m$, temos

$$
A^{\mu} B_{\mu}=\sum_{\mu=1}^{m} A^{\mu} B_{\mu}
$$

Um vetor dual é um objeto linear que aplica um vetor a um escalar. Isto pode ser generalizado para objetos multilineares, chamados tensores, que aplicam vários vetores e vetores duais a um escalar. Um tensor $T$ do tipo $(p, q)$ é uma aplicação multilinear que aplica $p$ vetores duais e $q$ vetores a um número real, ou seja,

$$
T: \underbrace{V^{*} \otimes \cdots \otimes V^{*}}_{p \text { vezes }} \otimes \underbrace{V \otimes \cdots \otimes V}_{q \text { vezes }} \rightarrow \mathbb{R} .
$$

Por exemplo, um tensor do tipo $(0,1)$ aplica um vetor a um número real e está identificado com um vetor dual. Analogamente, um tensor do tipo $(1,0)$ é um vetor. Se $\omega$ aplica um vetor dual e dois vetores a um escalar, então $\omega: V^{*} \times V \times V \rightarrow \mathbb{R}$ é do tipo $(1,2)$.

O conjunto de todos os tensores do tipo $(p, q)$ é chamado o espaço tensor do tipo $(p, q)$ e denotado por $\mathcal{T}_{q}^{p}$. O produto tensorial $\tau=\mu \otimes \nu \in \mathcal{T}_{q}^{p} \otimes \mathcal{T}_{q^{\prime}}^{p^{\prime}}$ é um elemento de $\mathcal{T}_{q+q^{\prime}}^{p+p^{\prime}}$ definido por

$$
\begin{aligned}
& \tau\left(\omega_{1}, \ldots, \omega_{p}, \xi_{1}, \ldots, \xi_{p^{\prime}} ; u_{1}, \ldots, u_{q}, v_{1}, \ldots, v_{q^{\prime}}\right)= \\
& \quad=\mu\left(\omega_{1}, \ldots, \omega_{p} ; u_{1}, \ldots, u_{q}\right) \nu\left(\xi_{1}, \ldots, \xi_{p^{\prime}} ; v_{1}, \ldots, v_{q^{\prime}}\right) .
\end{aligned}
$$

B.1 DEFINIÇÃo: Uma forma diferencial de ordem $r$, ou uma $r$-forma, é um tensor totalmente anti-simétrico do tipo $(0, r)$. 
Definimos o produto 'wedge' $\wedge$ de $r$ 1-formas pelo produto tensorial totalmente anti-simétrico

$$
d x^{\mu_{1}} \wedge d x^{\mu_{2}} \wedge \cdots \wedge d x^{\mu_{r}}=\sum_{\sigma \in S_{r}} \operatorname{sgn}(\sigma) d x^{\mu_{\sigma(1)}} \otimes d x^{\mu_{\sigma(2)}} \otimes \cdots \otimes d x^{\mu_{\sigma(r)}} .
$$

Por exemplo,

$$
\begin{aligned}
d x^{\mu} \wedge d x^{\nu} & =\quad d x^{\mu} \otimes d x^{\nu}-d x^{\nu} \otimes d x^{\mu} \\
d x^{\lambda} \wedge d x^{\mu} \wedge d x^{\nu}= & d x^{\lambda} \otimes d x^{\mu} \otimes d x^{\nu}+d x^{\nu} \otimes d x^{\lambda} \otimes d x^{\mu}+d x^{\mu} \otimes d x^{\nu} \otimes d x^{\lambda} \\
& -d x^{\lambda} \otimes d x^{\nu} \otimes d x^{\mu}-d x^{\nu} \otimes d x^{\mu} \otimes d x^{\lambda}-d x^{\mu} \otimes d x^{\lambda} \otimes d x^{\nu} .
\end{aligned}
$$

Rapidamente se verifica que o produto 'wedge' satisfaz:

1. $d x^{\mu_{1}} \wedge \cdots \wedge d x^{\mu_{r}}=0$, se algum índice $\mu_{i}$ aparece pelo menos duas vezes.

2. $d x^{\mu_{1}} \wedge \cdots \wedge d x^{\mu_{r}}=\operatorname{sgn}(\sigma) d x^{\mu_{\sigma(1)}} \wedge \cdots \wedge d x^{\mu_{\sigma(r)}}$.

3. $d x^{\mu_{1}} \wedge \cdots \wedge d x^{\mu_{r}}$ é linear em cada $d x^{\mu_{i}}$.

Se denotarmos o espaço vetorial das $r$-formas em $p \in M^{m}$, por $\Omega_{p}^{r}(M)$, o conjunto das $r$-formas (B.1) forma uma base para $\Omega_{p}^{r}(M)$ e um elemento $\omega \in \Omega_{p}^{r}(M)$ é escrito como

$$
\omega=\frac{1}{r !} \omega_{\mu_{1} \mu_{2} \ldots \mu_{r}} d x^{\mu_{1}} \wedge d x^{\mu_{2}} \wedge \cdots \wedge d x^{\mu_{r}}
$$

onde $\omega_{\mu_{1} \mu_{2} \ldots \mu_{r}}$ são totalmente anti-simétricos, refletindo a anti-simetria da base.

Desde que existem $\left(\begin{array}{c}m \\ r\end{array}\right)$ escolhas do conjunto $\left(\mu_{1}, \mu_{2}, \ldots, \mu_{r}\right)$, a dimensão do espaço vetorial $\Omega_{p}^{r}(M)$ é

$$
\left(\begin{array}{c}
m \\
r
\end{array}\right)=\frac{m !}{r !(m-r) !}
$$

Por conveniência, definimos $\Omega_{p}^{0}(M)=\mathbb{R}$. Claramente $\Omega_{p}^{1}(M)=T_{p}^{*}(M)$. Se $r$ em (B.1) excede $m$, o espaço se anula desde que algum índice aparece no mínimo duas vezes no somatório. A igualdade $\left(\begin{array}{c}m \\ r\end{array}\right)=\left(\begin{array}{c}m \\ m-r\end{array}\right)$ implica $\operatorname{dim} \Omega_{p}^{r}(M)=\operatorname{dim} \Omega_{p}^{m-r}(M)$. Desde que $\Omega_{p}^{r}(M)$ é um espaço vetorial, $\Omega_{p}^{r}(M)$ é isomorfo a $\Omega_{p}^{m-r}(M)$.

Definimos o produto exterior de uma $q$-forma e uma $r$-forma, $\wedge: \Omega_{p}^{q}(M) \times \Omega_{p}^{r}(M) \rightarrow \Omega_{p}^{q+r}(M)$, por uma extensão trivial. Sejam $\omega \in \Omega_{p}^{q}(M)$ e $\xi \in \Omega_{p}^{r}(M)$. A ação da $(q+r)$-forma $\omega \wedge \xi$ em $q+r$ vetores é definida por

$$
(\omega \wedge \xi)\left(v_{1}, \ldots, v_{q+r}\right)=\frac{1}{q ! r !} \sum_{\sigma \in S_{q+r}} \operatorname{sgn}(\sigma) \omega\left(v_{\sigma(1)}, \ldots, v_{\sigma(q)}\right) \xi\left(v_{\sigma(q+1)}, \ldots, v_{\sigma(q+r)}\right)
$$

onde $v_{i} \in T_{p}(M)$. Se $q+r>m, \omega \wedge \xi$ é identicamente nula. Com este produto definimos uma álgebra

$$
\Omega_{p}^{*}(M)=\Omega_{p}^{0}(M) \otimes \Omega_{p}^{1}(M) \otimes \cdots \otimes \Omega_{p}^{m}(M)
$$


$\Omega_{p}^{*}(M)$ é o espaço de todas as formas diferenciais em $p$ e é fechado pelo produto exterior.

Podemos associar suavemente uma $r$-forma a cada ponto de uma variedade $M$. Denotamos o espaço das $r$-formas suaves em $M$ por $\Omega^{r}(M)$. Também definimos $\Omega^{0}(M)$ sendo a álgebra das funções suaves, $C^{\infty}(M)$. Em resumo, temos a seguinte tabela:

\begin{tabular}{lcc}
\hline$r$-formas & Base & Dimensão \\
\hline$\Omega^{0}(M)=C^{\infty}(M)$ & $\{1\}$ & 1 \\
$\Omega^{1}(M)=T^{*}(M)$ & $\left\{d x^{\mu}\right\}$ & $m$ \\
$\Omega^{2}(M)$ & $\left\{d x^{\mu_{1}} \wedge d x^{\mu_{2}}\right\}$ & $m(m-1) / 2$ \\
\multicolumn{1}{c}{$\vdots$} & $\vdots$ & $\vdots$ \\
$\Omega^{m}(M)$ & $\left\{d x^{1} \wedge d x^{2} \wedge \cdots \wedge d x^{m}\right\}$ & 1 \\
\hline
\end{tabular}

B.2 DEFINIÇÃo: A derivada exterior $d_{r}$ é uma aplicação $d_{r}: \Omega^{r}(M) \rightarrow \Omega^{r+1}(M)$ cuja ação em uma $r$-forma

$$
\omega=\frac{1}{r !} \omega_{\mu_{1} \mu_{2} \ldots \mu_{r}} d x^{\mu_{1}} \wedge d x^{\mu_{2}} \wedge \cdots \wedge d x^{\mu_{r}}
$$

é definida por

$$
d_{r} \omega=\frac{1}{r !}\left(\frac{\partial}{\partial x^{\nu}} \omega_{\mu_{1} \ldots \mu_{r}}\right) d x^{\nu} \wedge d x^{\mu_{1}} \wedge d x^{\mu_{2}} \wedge \cdots \wedge d x^{\mu_{r}} .
$$

B.3 EXEMPLO: As $r$-formas em um espaço tridimensional são

$$
\begin{aligned}
& \omega_{0}=f(x, y, z) \\
& \omega_{1}=\omega_{x}(x, y, z) d x+\omega_{y}(x, y, z) d y+\omega_{z}(x, y, z) d z, \\
& \omega_{2}=\omega_{x y}(x, y, z) d x \wedge d y+\omega_{y z}(x, y, z) d y \wedge d z,+\omega_{z x}(x, y, z) d z \wedge d x \\
& \omega_{3}=\omega_{x y z}(x, y, z) d x \wedge d y \wedge d z .
\end{aligned}
$$

A ação de $d_{r}$ nestas formas é

$$
\begin{aligned}
d_{0} \omega_{0}= & \partial_{x} f(x, y, z) d x+\partial_{y} f(x, y, z) d y+\partial_{z} f(x, y, z) d z \\
d_{1} \omega_{1}= & \left(\partial_{x} \omega_{y}(x, y, z)-\partial_{y} \omega_{x}(x, y, z)\right) d x \wedge d y \\
& +\left(\partial_{y} \omega_{z}(x, y, z)-\partial_{z} \omega_{y}(x, y, z)\right) d y \wedge d z \\
& +\left(\partial_{z} \omega_{x}(x, y, z)-\partial_{x} \omega_{z}(x, y, z)\right) d z \wedge d x, \\
d_{2} \omega_{2}= & \left(\partial_{x} \omega_{y z}(x, y, z)+\partial_{y} \omega_{z x}(x, y, z)+\partial_{z} \omega_{x y}(x, y, z)\right) d x \wedge d y \wedge d z, \\
d_{3} \omega_{3}= & 0 .
\end{aligned}
$$

Portanto, a ação de $d$ em $\omega_{0}$ é identificada com 'grad', em $\omega_{1}$ com 'rot' e em $\omega_{2}$ com 'div', como no Cálculo Diferencial e Integral. 
B.4 Proposição: $d^{2}=0\left(\right.$ ou $\left.d_{r+1} d_{r}=0\right)$.

Prova: Seja $\omega=\frac{1}{r !} \omega_{\mu_{1} \mu_{2} \ldots \mu_{r}} d x^{\mu_{1}} \wedge d x^{\mu_{2}} \wedge \cdots \wedge d x^{\mu_{r}} \in \Omega^{r}(M)$. A ação de $d^{2}$ em $\omega$ é

$$
d^{2} \omega=\frac{1}{r !}\left(\frac{\partial^{2} \omega_{\mu_{1} \mu_{2} \ldots \mu_{r}}}{\partial x^{\lambda} \partial x^{\nu}}\right) d x^{\lambda} \wedge d x^{\nu} \wedge d x^{\mu_{1}} \wedge d x^{\mu_{2}} \wedge \cdots \wedge d x^{\mu_{r}} .
$$

Assim, $d^{2} \omega$ é identicamente nulo, desde que $\partial^{2} \omega_{\mu_{1} \mu_{2} \ldots \mu_{r}} / \partial x^{\lambda} \partial x^{\nu}$ é simétrico com relação a $\lambda$ e $\nu$ enquanto que $d x^{\lambda} \wedge d x^{\nu}$ é anti-simétrico.

A derivada exterior $d_{r}$ induz a seqüência

$$
0 \longrightarrow \Omega^{0}(M) \stackrel{d_{0}}{\longrightarrow} \Omega^{1}(M) \stackrel{d_{1}}{\longrightarrow} \cdots \stackrel{d_{m-2}}{\longrightarrow} \Omega^{m-1}(M) \stackrel{d_{m-1}}{\longrightarrow} \Omega^{m}(M) \stackrel{d_{m}}{\longrightarrow} 0 .
$$

Esta seqüência é chamada de complexo de de Rham. Desde que $d^{2}=0$, temos im $d_{r} \subseteq \operatorname{ker} d_{r+1}$. Um elemento de ker $d_{r}$ é chamado $r$-forma fechada, enquanto um elemento de im $d_{r-1}$ é chamado $r$-forma exata. O espaço quociente $\operatorname{ker} d_{r} / \mathrm{im} d_{r-1}$ é chamado de $r$-ésimo grupo de cohomologia de de Rham.

\section{B.2 Geometria Riemanniana}

Uma variedade é um espaço topológico que é localmente homeomorfo ao $\mathbb{R}^{n}$, para algum $n$. O cálculo em uma variedade é garantido pela existência de um sistema de coordenadas suaves. Uma variedade pode carregar uma outra estrutura se estiver equipada com um tensor métrico, que é uma generalização natural do produto interno entre dois vetores do $\mathbb{R}^{n}$, para uma variedade arbitrária.

$\mathrm{Na}$ geometria elementar, o produto interno entre dois vetores $u, v$ é definido por $\langle u, v\rangle=$ $\sum_{i=1}^{m} u_{i} v_{i}$, onde $u_{i}, v_{i}$ são as componentes dos vetores em $\mathbb{R}^{m}$. Em uma variedade, um produto interno é definido em cada espaço tangente $T_{p}(M)$.

B.5 DEFINIÇÃo: Seja $M$ uma variedade diferenciável. Uma métrica Riemanniana $g$ em $M$ é um tensor do tipo $(0,2)$ em $M$ que satisfaz o seguinte, para cada ponto $p \in M$ :

1. $g_{p}(u, v)=g_{p}(v, u)$.

2. $g_{p}(u, u) \geq 0$, onde a igualdade vale somente quando $u=0$.

Aqui, $u, v \in T_{p}(M)$ e $g_{p}=g_{\mid p}$. Em resumo, $g_{p}$ é uma forma bilinear simétrica positiva-definida. $\mathrm{O}$ par $(M, g)$ é chamado de variedade Riemanniana. 
Temos definido o produto interno entre um vetor $v \in T_{p}(M)$ e um vetor dual $\omega \in T_{p}^{*}(M)$ como sendo um aplicação $\langle\rangle:, T_{p}^{*}(M) \times T_{p}(M) \rightarrow \mathbb{R}$. Se existe uma métrica $g$, definimos um produto interno entre dois vetores $u, v \in T_{p}(M)$ por $g_{p}(u, v)$. Desde que $g_{p}$ é uma aplicação $T_{p}(M) \otimes T_{p}(M) \rightarrow \mathbb{R}$, podemos definir uma aplicação linear $g_{p}(u):, T_{p}(M) \rightarrow \mathbb{R}$ por $v \mapsto g_{p}(u, v)$. Então $g_{p}(u$,$) é identificada com uma 1-forma \omega_{u} \in T_{p}^{*}(M)$. Analogamente, $\omega \in T_{p}^{*}(M)$ induz $v_{\omega} \in T_{p}(M)$ por $\langle\omega, u\rangle=g_{p}\left(v_{\omega}, u\right)$. Assim, a métrica $g_{p}$ dá origem a um isomorfismo entre $T_{p}(M)$ e $T_{p}^{*}(M)$.

Sejam $(U, \phi)$ uma carta em $M$ e $\left\{x^{\mu}\right\}$ as coordenadas. Desde que $g \in \mathcal{T}_{2}^{0}(M), g$ é expandida em termos de $d x^{\mu} \otimes d x^{\nu}$ como

$$
g_{p}=g_{\mu \nu}(p) d x^{\mu} \otimes d x^{\nu}
$$

Facilmente verifica-se que

$$
g_{\mu \nu}(p)=g_{p}\left(\partial / \partial x^{\mu}, \partial / \partial x^{\nu}\right)=g_{\nu \mu}(p)
$$

Geralmente omitimos $p$ em $g_{\mu \nu}$, a menos que possa causar confusão. É comum tratar $\left(g_{\mu \nu}\right)$ como uma matriz cuja entrada $(\mu, \nu)$ é $g_{\mu \nu}$. Desde que $\left(g_{\mu \nu}\right)$ tem 'rank' maximal, $\left(g_{\mu \nu}\right)$ tem uma inversa denotada por $\left(g^{\mu \nu}\right)$, satisfazendo $g_{\mu \nu} g^{\nu \lambda}=g^{\lambda \nu} g_{\nu \mu}=\delta_{\mu}^{\lambda}$. O determinante $\operatorname{det}\left(g_{\mu \nu}\right)$ é denotado por $g \operatorname{edet}\left(g^{\mu \nu}\right)=g^{-1}$. O isomorfismo entre $T_{p}(M)$ e $T_{p}^{*}(M)$ pode agora ser expresso como

$$
\omega_{\mu}=g_{\mu \nu} u^{\nu} \quad u^{\mu}=g^{\mu \nu} \omega_{\nu}
$$

De (B.2) e (B.3) reescrevemos a definição de uma métrica como o quadrado de uma distância infinitesimal

$$
d s^{2}=g\left(\partial / \partial x^{\mu} d x^{\mu}, \partial / \partial x^{\nu} d x^{\nu}\right)=g\left(\partial / \partial x^{\mu}, \partial / \partial x^{\nu}\right) d x^{\mu} d x^{\nu}=g_{\mu \nu} d x^{\mu} d x^{\nu}
$$

Também chamamos a quantidade $d s^{2}=g_{\mu \nu} d x^{\mu} d x^{\nu}$ de métrica, embora em um sentido mais estrito ela seja um tensor $g=g_{\mu \nu} d x^{\mu} \otimes d x^{\nu}$. Desde que $\left(g_{\mu \nu}\right)$ é uma matriz real e simétrica, seus autovalores são todos reais e positivos. ${ }^{1}$

Seja $M$ uma subvariedade de dimensão $m$ de uma variedade Riemanniana $N$ de dimensão $n$, com uma métrica $g_{N}$. Se $f: M \rightarrow N$ é um mergulho que induz a estrutura de subvariedade de $M$, o 'pullback' $f^{*}$ induz a métrica natural $g_{M}=f^{*} g_{N}$ em $M$. As componentes de $g_{M}$ são dadas por

$$
g_{M \mu \nu}(x)=g_{N \alpha \beta}(f(x)) \frac{\partial f^{\alpha}}{\partial x^{\mu}} \frac{\partial f^{\beta}}{\partial x^{\nu}}
$$

onde $f^{\alpha}$ denota as coordenadas de $f(x)$. Por exemplo, considere a métrica da esfera unitária mergulhada em $\left(\mathbb{R}^{3}, g_{E}\right)$. Seja $(\theta, \phi)$ a coordenada polar de $S^{2}$ e defina $f$ pela inclusão usual

$$
f:(\theta, \phi) \mapsto(\operatorname{sen} \theta \cos \phi, \operatorname{sen} \theta \operatorname{sen} \phi, \cos \theta),
$$

\footnotetext{
${ }^{1}$ Para uma métrica pseudo-Riemanniana podem haver autovalores negativos.
} 
da qual obtemos a métrica induzida

$$
\begin{aligned}
g_{\mu \nu} d x^{\mu} \otimes d x^{\nu} & =g_{E \alpha \beta} \frac{\partial f^{\alpha}}{\partial x^{\mu}} \frac{\partial f^{\beta}}{\partial x^{\nu}} d x^{\mu} \otimes d x^{\nu} \\
& =d \theta \otimes d \theta+(\operatorname{sen} \theta)^{2} d \phi \otimes d \phi .
\end{aligned}
$$

\section{B.3 Teoria de Hodge}

Se $M$ é uma variedade diferenciável orientável de dimensão $m$, existe uma $m$-forma $\omega$ que não se anula em ponto algum. Esta $m$-forma é chamada elemento volume (ou forma de volume) e faz o papel de uma medida quando integramos uma função $f \in C^{\infty}(M)$ sobre $M$. Se $M$ possui uma métrica $g$, existe um elemento volume natural que é invariante por transformações coordenadas. Definimos o elemento volume invariante por

$$
\Omega_{M}=\sqrt{|g|} d x^{1} \wedge d x^{2} \wedge \cdots \wedge d x^{m}
$$

onde $g=\operatorname{det}\left(g_{\mu \nu}\right)$ e $\left\{x^{\mu}\right\}$ são as coordenadas da carta $(U, \phi)$. A $m$-forma $\Omega_{M}$ é, de fato, invariante por uma mudança de coordenada. Sejam $\left\{y^{\lambda}\right\}$ as coordenadas da carta $(V, \psi)$, com $U \cap V \neq \emptyset$. Em termos da coordenada $y$, o elemento volume invariante é

$$
\left|\operatorname{det}\left(\frac{\partial x^{\mu}}{\partial y^{\kappa}} \frac{\partial x^{\nu}}{\partial y^{\lambda}} g_{\mu \nu}\right)\right|^{1 / 2} d y^{1} \wedge \cdots \wedge d y^{m} .
$$

Notando que $d y^{\lambda}=\partial y^{\lambda} / \partial x^{\mu} d x^{\mu}$, isto torna-se

$$
\left|\operatorname{det}\left(\partial x^{\mu} / \partial y^{\kappa}\right)\right| \sqrt{|g|} \operatorname{det}\left(\partial y^{\lambda} / \partial x^{\nu}\right) d x^{1} \wedge \cdots \wedge d x^{m}= \pm \sqrt{|g|} d x^{1} \wedge \cdots \wedge d x^{m} .
$$

Se $x$ e $y$ definem a mesma orientação, $\operatorname{det}\left(\partial x^{\mu} / \partial y^{\kappa}\right)$ é estritamente positivo em $U \cap V$ e $\Omega_{M}$ é invariante pela mudança de coordenada.

Como notado anteriormente, $\Omega^{r}(M)$ é isomorfo a $\Omega^{m-r}(M)$, para uma variedade $M$ de dimensão $m$. Se $M$ possui uma métrica $g$, podemos definir um isomorfismo natural entre $\Omega^{r}(M)$ e $\Omega^{m-r}(M)$, chamado $\star$ de Hodge. Para isto, definimos o tensor totalmente anti-simétrico $\varepsilon$ por

$$
\varepsilon_{\mu_{1} \mu_{2} \ldots \mu_{m}}=\left\{\begin{array}{cl}
+1 & \text { se }\left(\mu_{1} \mu_{2} \ldots \mu_{m}\right) \text { é uma permutação par de }(12 \ldots m), \\
-1 & \text { se }\left(\mu_{1} \mu_{2} \ldots \mu_{m}\right) \text { é uma permutação ímpar de }(12 \ldots m), \\
0 & \text { caso contrário. }
\end{array}\right.
$$

Note que $\varepsilon^{\mu_{1} \mu_{2} \ldots \mu_{m}}=g^{\mu_{1} \nu_{1}} g^{\mu_{2} \nu_{2}} \cdots g^{\mu_{m} \nu_{m}} \varepsilon_{\nu_{1} \nu_{2} \ldots \nu_{m}}=g^{-1} \varepsilon_{\mu_{1} \mu_{2} \ldots \mu_{m}}$.

$\mathrm{A} \star$ de Hodge é uma aplicação linear $\star: \Omega^{r}(M) \rightarrow \Omega^{m-r}(M)$ cuja ação em um vetor da base de $\Omega^{r}(M)$ é definida por

$$
\star\left(d x^{\mu_{1}} \wedge \cdots \wedge d x^{\mu_{r}}\right)=\frac{\sqrt{|g|}}{(m-r) !} \varepsilon_{\nu_{r+1} \ldots \nu_{m}}^{\mu_{1} \ldots \mu_{r}} d x^{\nu_{r+1}} \wedge \cdots \wedge d x^{\nu_{m}} .
$$


Notemos que $\star 1$ é o elemento volume invariante

$$
\star 1=\frac{\sqrt{|g|}}{m !} \varepsilon_{\mu_{1} \ldots \mu_{m}} d x^{\mu_{1}} \wedge \cdots \wedge d x^{\mu_{m}}=\sqrt{|g|} d x^{\mu_{1}} \wedge \cdots \wedge d x^{\mu_{m}} .
$$

Para

$$
\omega=\frac{1}{r !} \omega_{\mu_{1} \mu_{2} \ldots \mu_{r}} d x^{\mu_{1}} \wedge d x^{\mu_{2}} \wedge \cdots \wedge d x^{\mu_{r}} \in \Omega^{r}(M)
$$

temos

$$
\star \omega=\frac{\sqrt{|g|}}{r !(m-r) !} \omega_{\mu_{1} \ldots \mu_{r}} \varepsilon^{\mu_{1} \ldots \mu_{r}}{ }_{\nu_{r+1} \ldots \nu_{m}} d x^{\nu_{r+1}} \wedge \cdots \wedge d x^{\nu_{m}} .
$$

B.6 TEOREMA: Sejam $(M, g)$ uma variedade Riemanniana e $\omega \in \Omega^{r}(M)$. Então

$$
\star \star \omega=(-1)^{r(m-r)} \omega .
$$

Assim, encontramos que $(-1)^{r(m-r)} \star \star$ é a identidade em $\Omega^{r}(M)$. Definimos a inversa de $\star$ por

$$
\star^{-1}=(-1)^{r(m-r)} \star .
$$

Sejam $\omega, \eta \in \Omega^{r}(M)$ dadas por

$$
\begin{aligned}
& \omega=\frac{1}{r !} \omega_{\mu_{1} \mu_{2} \ldots \mu_{r}} d x^{\mu_{1}} \wedge d x^{\mu_{2}} \wedge \cdots \wedge d x^{\mu_{r}}, \\
& \eta=\frac{1}{r !} \eta_{\mu_{1} \mu_{2} \ldots \mu_{r}} d x^{\mu_{1}} \wedge d x^{\mu_{2}} \wedge \cdots \wedge d x^{\mu_{r}} .
\end{aligned}
$$

O produto exterior $\omega \wedge \star \eta$ é uma $m$-forma,

$$
\begin{aligned}
\omega \wedge \star \eta & =\frac{1}{(r !)^{2}} \omega_{\mu_{1} \ldots \mu_{r}} \eta_{\nu_{1} \ldots \nu_{r}} \frac{\sqrt{|g|}}{(m-r) !} \varepsilon^{\nu_{1} \ldots \nu_{r}}{ }_{\mu_{r+1} \ldots \mu_{m}} d x^{\mu_{1}} \wedge \cdots \wedge d x^{\mu_{r}} \wedge d x^{\mu_{r+1}} \wedge \cdots \wedge d x^{\mu_{m}} \\
& =\frac{1}{r !} \sum_{\mu, \nu} \omega_{\mu_{1} \ldots \mu_{r}} \eta^{\nu_{1} \ldots \nu_{r}} \frac{1}{r !(m-r) !} \varepsilon_{\nu_{1} \ldots \nu_{r} \mu_{r+1} \ldots \mu_{m}} \varepsilon_{\mu_{1} \ldots \mu_{r} \mu_{r+1} \ldots \mu_{m}} \sqrt{|g|} d x^{1} \wedge \cdots \wedge d x^{m} \\
& =\frac{1}{r !} \omega_{\mu_{1} \ldots \mu_{r}} \eta^{\mu_{1} \ldots \mu_{r}} \sqrt{|g|} d x^{1} \wedge \cdots \wedge d x^{m}
\end{aligned}
$$

Esta expressão mostra que o produto é simétrico, ou seja, $\omega \wedge \star \eta=\eta \wedge \star \omega$. Desde que $\omega \wedge \star \eta$ é uma $m$-forma, sua integral sobre $M$ está bem definida. Assim, definimos o produto interno $(\omega, \eta)$ de duas $r$-formas por

$$
(\omega, \eta)=\int \omega \wedge \star \eta=\frac{1}{r !} \int_{M} \omega_{\mu_{1} \ldots \mu_{r}} \eta^{\mu_{1} \ldots \mu_{r}} \sqrt{|g|} d x^{1} \wedge \ldots \wedge d x^{m} .
$$

Desde que $\omega \wedge \star \eta=\eta \wedge \star \omega$, o produto interno é simétrico, $(\omega, \eta)=(\eta, \omega)$. Se $(M, g)$ é Riemanniana, o produto interno é positivo definido, isto é, $(\omega, \omega) \geq 0$, onde a igualdade é válida somente se $\omega=0$. 
B.7 DEFINIÇÃo: Seja $(M, g)$ uma variedade Riemanniana de dimensão $m$, com derivada exterior $d: \Omega^{r-1}(M) \rightarrow \Omega^{r}(M)$. O operador derivada exterior adjunto $d^{\dagger}: \Omega^{r}(M) \rightarrow \Omega^{r-1}(M)$ é definido por

$$
d^{\dagger}=(-1)^{m(r+1)+1} \star d \star .
$$

Um elemento de ker $d^{\dagger}$ é chamado de $r$-forma cofechada e um elemento de im $d^{\dagger}$ é chamado de $r$-forma coexata.

Em resumo, temos o seguinte diagrama (a menos de $\left.(-1)^{m(r+1)+1}\right)$ :

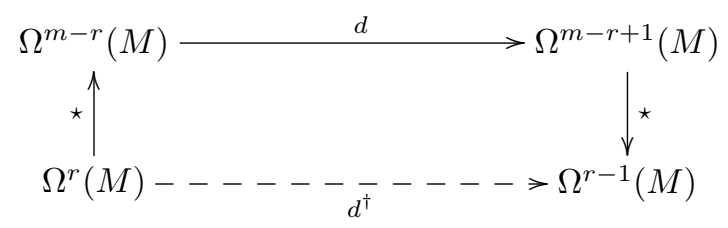

O operador $d^{\dagger}$ é nilpotente pois $\left(d^{\dagger}\right)^{2}=\star d \star \star d \star= \pm \star d^{2} \star=0$.

Seja $\Omega^{r}(M)$ o conjunto das $r$-formas diferenciais sobre a variedade $M$. Existe um complexo de cadeias e um operador bordo $d$, bem como um complexo de cocadeias e um operador cobordo $d^{\dagger}$, isto é,

$$
\cdots \leftrightarrows \Omega^{3}(M) \stackrel{d_{2}}{\underset{d_{3}^{\dagger}}{\leftrightarrows}} \Omega^{2}(M) \stackrel{d_{1}}{\stackrel{d_{2}^{\dagger}}{\leftrightarrows}} \Omega^{1}(M) \stackrel{d_{0}}{\stackrel{d_{1}^{\dagger}}{\leftrightarrows}} \Omega^{0}(M) \leftrightarrows 0
$$

e definimos o Laplaciano

$$
\Delta_{r}=d_{r+1}^{\dagger} d_{r}+d_{r-1} d_{r}^{\dagger}: \Omega^{r}(M) \rightarrow \Omega^{r}(M)
$$

Note que o Laplaciano comuta com o operador (co)bordo, isto é, o diagrama abaixo comuta,

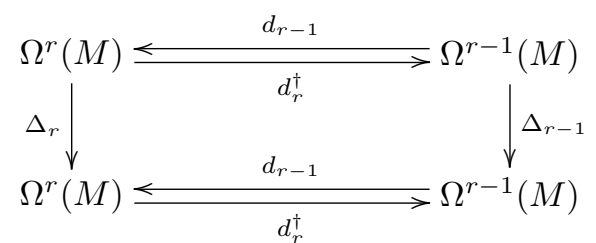

pois, como $d^{2}=0\left(\left(d^{\dagger}\right)^{2}=0\right)$ temos

$$
\begin{aligned}
d_{r-1} \Delta_{r-1}=d_{r-1}\left(d_{r}^{\dagger} d_{r-1}+\right. & \left.d_{r-2} d_{r-1}^{\dagger}\right)=d_{r-1} d_{r}^{\dagger} d_{r-1}= \\
& =d_{r+1}^{\dagger} d_{r} d_{r-1}+d_{r-1} d_{r}^{\dagger} d_{r-1}=\left(d_{r+1}^{\dagger} d_{r}+d_{r-1} d_{r}^{\dagger}\right) d_{r-1}=\Delta_{r} d_{r-1}
\end{aligned}
$$

Analogamente para $\Delta_{r-1} d_{r}^{\dagger}=d_{r}^{\dagger} \Delta_{r}$.

Assim, se $\omega \in \Omega^{r}(M)$ é autoforma de $\Delta_{r}$, isto é, $\Delta_{r}(\omega)=\lambda \omega$ para algum $\lambda \in \mathbb{R}$, então $d_{r} \omega$ é autoforma de $\Delta_{r+1}$, pois

$$
\Delta_{r+1}\left(d_{r} \omega\right)=d_{r} \Delta_{r}(\omega)=d_{r} \lambda \omega=\lambda d_{r} \omega
$$


Seja $(M, g)$ uma variedade Riemanniana compacta. O Laplaciano $\Delta$ é um operador positivo em $M$ no sentido de que

$$
(\omega, \Delta \omega)=\left(\omega,\left(d d^{\dagger}+d^{\dagger} d\right) \omega\right)=(d \omega, d \omega)+\left(d^{\dagger} \omega, d^{\dagger} \omega\right) \geq 0
$$

Uma forma $\omega$ é chamada harmônica se $\Delta \omega=0$. Denotamos o conjunto das $r$-formas harmônicas em $M$ por $\mathcal{H}^{r}(M)$. Como conseqüência imediata de (B.4), temos:

B.8 ProposiçÃo: Uma forma $\omega$ sobre uma variedade compacta é harmônica se, e somente se, $\omega$ é fechada e cofechada.

B.9 TEOREMA (Decomposição de Hodge): Seja $(M, g)$ uma variedade Riemanniana orientável compacta sem bordo. Então $\Omega^{r}(M)$ é unicamente decomposto como

$$
\Omega^{r}(M)=\mathcal{H}^{r}(M) \oplus d_{r-1} \Omega^{r-1}(M) \oplus d_{r+1}^{\dagger} \Omega^{r+1}(M),
$$

ou seja, qualquer $r$-forma $\omega_{r}$ é escrita globalmente como

$$
\omega_{r}=\gamma_{r}+d_{r-1} \alpha_{r-1}+d_{r+1}^{\dagger} \beta_{r+1}
$$

$\operatorname{com} \alpha_{r-1} \in \Omega^{r-1}(M), \beta_{r+1} \in \Omega^{r+1}(M)$ e $\gamma_{r} \in \mathcal{H}^{r}(M)$

O operador $\Delta_{r}$ deixa tal decomposição invariante. De fato, se $d_{r-1} \omega \in d_{r-1} \Omega^{r-1}(M)$ então

$$
\Delta_{r}\left(d_{r-1} \omega\right)=d_{r+1}^{\dagger} d_{r} d_{r-1} \omega+d_{r-1} d_{r}^{\dagger} d_{r-1} \omega=d_{r-1}\left(d_{r}^{\dagger} d_{r-1} \omega\right)=d_{r-1} \zeta
$$

onde $\zeta=d_{r}^{\dagger} d_{r-1} \omega \in \Omega^{r-1}(M)$. Analogamente para $d_{r+1}^{\dagger} \Omega^{r+1}(M)$. 



\section{LISTA DE TABELAS E FIGURAS}

\section{TABElAS}

2.1 Grupos livres de ponto fixo dos Tipos I e II . . . . . . . . . . . . . . . . . 27

2.2 Ação dos automorfismos de $G$ em $\mathfrak{F}_{\mathbb{C}}(G) \ldots \ldots \ldots \ldots$. . . . . . . . . . . 28

\section{FIGURAS}

1.1 Complexos $K, K^{\prime}=\operatorname{sd}(K)$ e $\hat{K}=\operatorname{dual}(K) \ldots \ldots \ldots \ldots \ldots$

3.1 Representação tridimensional para a esfera $S^{3}$. . . . . . . . . . . . . . . 35

3.2 Região fundamental $\mathcal{F}_{8,3}$. . . . . . . . . . . . . . . . . . . . . . . . 36

3.3 Decomposição celular da região fundamental $\mathcal{F}_{8,3}$. . . . . . . . . . . . . . . . . . 38

3.4 Região fundamental $\mathcal{F}_{4 t, 3} \ldots \ldots \ldots$. . . . . . . . . . . . . . . . . . 39

3.5 Arcos de referência da região fundamental $\mathcal{F}_{4 t, 3}$. . . . . . . . . . . . . . . . . . 39

3.6 Ação de $\mathcal{Q}_{16}$ em $\mathcal{F}_{16,3} \ldots \ldots \ldots$. . . . . . . . . . . . . . . . . . . . . . . 40

3.7 Decomposição celular da região fundamental $\mathcal{F}_{4 t, 3}$. . . . . . . . . . . . . . . . . . 41

3.8 Arcos de referência da região fundamental $\mathcal{F}_{16,7} \ldots \ldots \ldots$. . . . . . . . . . . . 42 



\section{LisTA DE SÍMBOLOS}

$(c / b)$

$\left(m_{i j}\right)$

$(\omega, \eta)$

$(v, w)$

$(K, L)$

$(W, g)$

$[c / b]$

$*$
$\tilde{*}$

$\star$

$\sim$

$\simeq$

$\approx$

$\equiv$

$\wedge$

$\mathfrak{A}_{\mathbb{C}}(G)$

$\mathfrak{F}_{\mathbb{C}}(G)$

$\theta$

$\zeta$

$\bar{\pi}$

$\pi^{*}$

$\pi_{k}$

$\pi_{k, l}$

$\hat{\pi}_{k, l}$

$\pi_{k}^{\mathcal{Q}_{4 t}}$

$\phi(n)$

$\sigma_{\mathbb{C}}$
Matriz da mudança da base $b$ para a base $c$

6

Matriz cujo elemento da linha $i$ e coluna $j$ é $m_{i j} \quad 21$

Produto interno entre as formas $\omega$ e $\eta \quad 13$

Produto interno entre os vetores $v$ e $w \quad 13$

Par de complexos celulares (ou simpliciais) 11

Variedade Riemanniana $W$ com métrica $g \quad$ xii

Classe da matriz $(c / b)$ no grupo de Whitehead re- 6

duzido $\bar{K}_{1}(R)$

'Join'

'Curved join', difere do 'join' * pois é construído 34

utilizando-se arcos, não segmentos

Operador de Hodge

Equivalência de homotopia

Isomorfismo

Equivalência ou congruência $\quad 35$

Produto exterior nos fibrados de de Rham das formas $\quad 13,68$

sobre uma variedade

Grupo das transformações de $\mathfrak{F}_{\mathbb{C}}(G)$ induzidas por au-

tomorfismos de $G$

Conjunto de todas as classes de equivalência de representações complexas irredutíveis livres de ponto fixo de $G$

Constante $\pi / t$, onde $t$ é o mesmo do grupo $\mathcal{Q}_{4 t}$

Raíz primitiva da unidade $\zeta=e^{i \theta}$

Representação conjugada da representação $\pi$

Representação dual da representação $\pi$

Representação induzida por $\sigma_{k}$

Representação, definida na equação (2.3)

Representação real obtida da representação $\pi_{k, l}$

Representação para o grupo $\mathcal{Q}_{4 t}$

Função $\phi$ de Euler. $\phi(n)$ é o número de geradores do grupo cíclico de ordem $n$

Representação complexa, extensão da representação real $\sigma$
34

13,72

66

5

46

28

13
1
1
ii

4
4
3,72
6
5
35
3,68

28

34,38

34, 38

21

19,21

23

24,27

25

33

23-25

20 


\begin{tabular}{|c|c|c|}
\hline SímbоLO & DESCRIÇÃO & PÁGina \\
\hline$\sigma_{\mathrm{F}}$ & $\begin{array}{l}\text { Representação sobre } \mathbb{F} \text {, extensão da representação } \sigma \\
\text { sobre } \mathbb{K}\end{array}$ & 20 \\
\hline$\sigma^{G}$ & $\begin{array}{l}\text { Representação de } G \text { induzida por } \sigma \text { (representação de } \\
\text { um subgrupo de } G \text { ) }\end{array}$ & 33 \\
\hline$\sigma_{k}$ & Representação, definida na equação (2.2) & 23,27 \\
\hline$\tau_{\mathrm{R}}$ & $\mathrm{R}$ torção ou torção de Reidemeister & 11,12 \\
\hline$\tau_{\mathrm{RS}}$ & RS torção ou torção de Ray-Singer & $\begin{array}{l}\text { xii, } 15,49 \\
52,58\end{array}$ \\
\hline$\tau_{\mathrm{W}}$ & W torção ou torção de Whitehead & 11 \\
\hline$\omega_{\text {norm } / \tan }$ & Componente normal/tangente da forma $\omega$ & 13 \\
\hline$\chi_{\pi}$ & Caractere da representação finita $\pi$ & 19,26 \\
\hline$\Delta$ & Operador Laplaciano $\Delta=d d^{\dagger}+d^{\dagger} d$ & 13 \\
\hline$\Omega_{p}^{r}(M)$ & Espaço vetorial das $r$-formas em $p \in M$ & 13 \\
\hline$\Omega\left(W, E_{\rho}\right)$ & $\begin{array}{l}\text { Espaço das formas diferenciais em } W \text { com valores no } \\
\text { fibrado } E_{\rho}\end{array}$ & 13 \\
\hline$\Sigma^{1}$ & $\begin{array}{l}\text { Polígono regular em } \mathbb{R}^{2} \text { com vértices } v_{q}=e^{2 \pi i q / p} \text {, para } \\
q=0,1,2, \ldots, p-1\end{array}$ & 61 \\
\hline$\Sigma_{j}$ & Cópia de $\Sigma^{1}$ na $j$-ésima cópia de $\mathbb{R}^{2}$ em $\mathbb{R}^{2 n}$ & 34,41 \\
\hline$\Sigma^{j}$ & 'Curved join' iterado $\Sigma_{1} \tilde{*} \cdots \tilde{*} \Sigma_{j}$ & 34 \\
\hline$a E_{i j}$ & $\begin{array}{l}\text { Matriz com } a \text { na entrada }(i, j), i \neq j \text {, e zero, caso } \\
\text { contrário }\end{array}$ & 4 \\
\hline $\mathcal{A}^{q}, \mathcal{A}_{q}$ & Aplicação de de Rham & 14,15 \\
\hline $\mathcal{A}_{q}^{\mathrm{abs} / \mathrm{rel}}$ & $\begin{array}{l}\text { Aplicação de de Rham com condições de contorno ab- } \\
\text { solutas/relativas }\end{array}$ & 15 \\
\hline$b \approx c$ & Base $b$ equivalente à base $c$, isto é, $[c / b]$ é trivial & 6 \\
\hline$B_{q}$ & Imagem do operador bordo $\partial_{q}$ & 11 \\
\hline$B_{\mathrm{abs} / \mathrm{rel}}$ & Condições de contorno absolutas/relativas & 13 \\
\hline$c^{*}$ & Cocadeia dual da cadeia $c$ & 14 \\
\hline $\mathcal{C}_{k}$ & Grupo cíclico de ordem $k$ & 27,33 \\
\hline$C((K, L) ; R)$ & Complexo de cadeias de $(K, L)$ com coeficientes em $R$ & 14 \\
\hline$d^{\dagger}$ & Operador derivada exterior adjunto & 16,74 \\
\hline $\operatorname{deg}(\pi)$ & Grau da representação $\pi$ & 19 \\
\hline $\operatorname{deg}(f)$ & Grau da aplicação $f$ & 65,66 \\
\hline & Função determinante & 4 \\
\hline$D_{l}^{m}$ & Disco $m$-dimensional de raio $l>0$ & 56 \\
\hline$D_{ \pm}^{m-1}$ & Hemisfério superior/inferior do bordo $\partial D^{m}$ & 62 \\
\hline$E_{\rho}$ & Fibrado vetorial real associado à representação $\rho$ & 13 \\
\hline$E(R)$ & $\begin{array}{l}\text { Subgrupo de } G L(R) \text { gerado por todas as matrizes ele- } \\
\text { mentares }\end{array}$ & 3 \\
\hline
\end{tabular}




\begin{tabular}{|c|c|c|}
\hline SímbOLO & DESCRIÇÃO & PÁgina \\
\hline $\mathcal{E}\left[L, L^{\prime}\right]$ & $\begin{array}{l}\text { Conjunto das classes de equivalência por homotopia de } \\
\text { equivalências de homotopia } f: L \rightarrow L^{\prime}\end{array}$ & 66 \\
\hline $\mathbb{F}$ & Corpo arbitrário & 19 \\
\hline $\mathcal{F}_{\mu, \nu}$ & Região fundamental para a ação de $\mathcal{Q}_{\mu}$ em $S^{\nu}$ & $\begin{array}{l}\text { xiii, } 34,39 \text {, } \\
45\end{array}$ \\
\hline$g_{E}$ & Métrica Riemanniana Euclidiana & 56 \\
\hline$G L(R)$ & Grupo linear geral infinito, união dos grupos $G L(n, R)$ & 3 \\
\hline$G L(n, R)$ & $\begin{array}{l}\text { Grupo linear geral de todas as matrizes } n \times n \text { não } \\
\text { singulares sobre } R\end{array}$ & 3,8 \\
\hline $\mathrm{H}$ & Conjunto dos quatérnios & 7 \\
\hline $\mathrm{H}_{1}$ & Conjunto dos quatérnios unitários & 8,10 \\
\hline$H_{q}$ & Grupo de homologia $Z_{q} / B_{q}$ & 11 \\
\hline $\mathcal{H}^{q}$ & Espaço das $q$-formas harmônicas & 14 \\
\hline $\mathcal{H}_{\mathrm{abs} / \mathrm{rel}}^{q}$ & $\begin{array}{l}\text { Espaço das } q \text {-formas harmônicas satisfazendo condi- } \\
\text { ções de contorno absolutas/relativas }\end{array}$ & 14 \\
\hline$I_{j}$ & Arcos de circunferência com vértices $\left\{v_{j}, x^{r_{j}} v_{j}\right\}$ & $34,36,43$ \\
\hline $\mathbf{I}(\pi, \psi)$ & Conjunto dos operadores 'intertwining' de $\pi$ e $\psi$ & 20 \\
\hline$J_{j}$ & Arcos de circunferência com vértices $\left\{v_{j}, y v_{j}\right\}$ & $34,36,43$ \\
\hline $\mathbb{K}$ & Corpo arbitrário & 20 \\
\hline$K_{j}$ & Arcos de circunferência com vértices $\left\{v_{j}, x y v_{j}\right\}$ & 36 \\
\hline$K_{1}(R)$ & Grupo de Whitehead de $R$ & 4 \\
\hline $\bar{K}_{1}(R)$ & Grupo de Whitehead reduzido de $R$ & 5 \\
\hline$L_{x}$ & $\begin{array}{l}L_{x}=1+x+x^{2}+\cdots+x^{2 t-1}, \text { soma de todas as potências } \\
\text { de } x \in \mathcal{Q}_{4 t}\end{array}$ & 39 \\
\hline$L\left(p ; q_{1}, \ldots, q_{n}\right)$ & Espaço lenticular $\Sigma^{2 n-1} / \mathcal{C}_{p}$ & 61 \\
\hline$M(n, R)$ & Anel de todas as matrizes $n \times n$ sobre o anel $R$ & 5,8 \\
\hline$N_{x}$ & $\begin{array}{l}N_{x}=1+x+x^{2}+\cdots+x^{t-1}, \text { soma de potências de } \\
x \in \mathcal{Q}_{4 t}\end{array}$ & 39 \\
\hline$O(n, R)$ & $\begin{array}{l}\text { Anel de todas as matrizes } n \times n \text { sobre } R \text {, com deter- } \\
\text { minante } \pm 1\end{array}$ & 6 \\
\hline $\mathcal{P}_{q}$ & Isomorfismo de Lefschetz-Poincaré & 14 \\
\hline$P_{x, y}$ & Soma de todos os elementos de $\mathcal{Q}_{4 t}, P_{x, y}=L_{x}(1+y)$ & 41 \\
\hline $\mathcal{Q}_{2^{a}}$ & Grupo dos quatérnios generalizado de ordem $2^{a}, a \geq 3$ & $\begin{array}{l}23,25,27 \\
28,32\end{array}$ \\
\hline
\end{tabular}




\begin{tabular}{|c|c|c|}
\hline Sі́мвоLO & DESCRIÇÃO & PÁGina \\
\hline $\mathcal{Q}_{4 t}$ & Grupo dos quatérnios generalizado de ordem $4 t, t \geq 2$ & 31 \\
\hline $\mathcal{Q}\left(2 t ; q_{1}, \ldots, q_{n}\right)$ & $\begin{array}{l}\text { Forma espacial esférica quaterniônica, quociente da es- } \\
\text { fera pela ação do grupo dos quatérnios generalizado }\end{array}$ & 46 \\
\hline $\operatorname{rk}(\rho)$ & 'Rank' da representação $\rho$ & 58 \\
\hline$R^{\times}$ & Unidades de $R$ & 4 \\
\hline$R(\theta)$ & $\begin{array}{l}\text { Matriz real } 2 \times 2 \text { da rotação de ângulo } \theta \text { no sentido } \\
\text { anti-horário }\end{array}$ & 25 \\
\hline$R G$ & Anel de grupos & 6 \\
\hline $\operatorname{sd}(K)$ & Subdivisão baricêntrica de $K$ & 14 \\
\hline$S_{l}^{n}$ & Esfera $n$-dimensional de raio $l>0$ & 49 \\
\hline$S K_{1}(R)$ & Grupo de Whitehead especial de $R$ & 4 \\
\hline$S L(R)$ & $\begin{array}{l}\text { Grupo linear especial, matrizes em } G L(R) \text { com deter- } \\
\text { minante } 1\end{array}$ & 4,10 \\
\hline $\operatorname{Sp}(\Delta)$ & Espectro do operador Laplaciano $\Delta$ & 16 \\
\hline $\operatorname{tr}(\pi(g))$ & Traço da matriz $\pi(g)$ & 19 \\
\hline$T$ & Torção analítica & 16 \\
\hline$T_{\mathrm{abs} / \mathrm{rel}}$ & Torção analítica absoluta/relativa & xii, 16,60 \\
\hline$V^{*}$ & Espaço dual do espaço vetorial $V$ & 19 \\
\hline$V_{\mathrm{F}}$ & $\begin{array}{l}\text { Extensão escalar } V_{\mathbb{F}}=V \otimes_{\mathbb{K}} \mathbb{F} \text {, sobre o corpo } \mathbb{F} \text {, do } \\
\text { espaço vetorial de dimensão finita } V \text { sobre o corpo } \mathbb{K}\end{array}$ & 20 \\
\hline $\mathrm{Wh}(G)$ & Grupo de Whitehead do anel $\mathbb{Z} G$ & 6 \\
\hline $\mathrm{Wh}(R G)$ & Grupo de Whitehead do anel $R G$ & 6 \\
\hline$Z_{q}$ & Núcleo do operador bordo $\partial_{q}$ & 11 \\
\hline
\end{tabular}




\section{REFERÊNCIAS BIBLIOGRÁFICAS}

[1] E. ARTin, Geometric algebra, New York, Interscience Publishers, (1957).

[2] H. Aslaksen, Quaternionic Determinants, The Mathematical Intelligencer, 18 no. 3 (1996), 57-65.

[3] J.-M. Bismut And W. Zhang, An extension of a theorem by Cheeger and Müller, Astérisque 205 (1992).

[4] R. Bott And L. W. Tu, Differential Forms in Algebraic Topology, GTM 82 (1982).

[5] J. BRÜNING AND X. MA, An anomaly formula for Ray-Singer metrics on manifolds with boundary, GAFA 16 (2006), 767-873.

[6] W. BuRnside, On a general property of finite irreducible groups of linear substitutions, Messenger of Mathematics, 35 (1905), 51-55.

[7] J. Cheeger, Analytic torsion and the heat equation, Ann. Math. 109 (1979), 259-322.

[8] J. Cheeger, Spectral geometry of singular Riemannian spaces, J. Diff. Geom. 18 (1983), $575-657$.

[9] M. M. Cohen, A Course in Simple-Homotopy Theory, GTM 10 (1973).

[10] X. DAi AND H. FAng, Analytic torsion and R-torsion for manifolds with boundary, Asian J. Math. 4 (2000), 695-714.

[11] T. De Melo, L. Hartmann and M. Spreafico, Reidemeister torsion and analytic torsion of discs, preprint (2008).

[12] T. De Melo And M. Spreafico, Reidemeister torsion and analytic torsion of spheres, preprint (2008).

[13] G. DE Rham, Complexes à automorphismes et homéomorphie différentiables, Ann. Inst. Fourier, Grenoble, 2 (1950), 51-67. 
[14] J. Dieudonné, Les determinants sur un corps non commutatif, Bull. Soc. Math. France 71 (1943), 27-45.

[15] W. Franz, Über die Torsion einer Überdeckung, J. Reine Angew. Math. 173 (1935), 245254.

[16] P. B. Gilkey, Invariance Theory, the Heat Equation, and the Atiyah-Singer Index Theorem, Studies in Advanced Mathematics, 2nd edition.

[17] K. W. Kwun And R. H. Szczarba, Product and sum theorems for Whitehead Torsion, Ann. of Math. 82 (1965), 183-190.

[18] J. Lott And M. Rothenberg, Analytic torsion for group actions, J. Differential Geom. 34 no. 2 (1991), 431-481.

[19] W. LÜCK, Analytic and topological torsion for manifolds with boundary and symmetry, J. Differential Geom. 37 (1993), 263-322.

[20] J. Milnor, A duality theorem for Reidemeister torsion, Ann. of Math. 76 (1962), 137-147.

[21] J. MiLnor, Whitehead torsion, Bull. AMS 72 (1966), 358-426.

[22] W. MÜLLER, Analytic torsion and R-torsion of Riemannian manifolds, Adv. Math. 28 (1978), 233-305.

[23] J. R. Munkres, Elements of Algebraic Topology, The Benjamim/cummings Publishing Company Inc (1993).

[24] M. NAKAhara, Geometry, topology and physics, Graduate student series in physics. Hadam Hilger (1990).

[25] R. OLIVER, Whitehead groups of finite groups, London Mathematical Society. Lecture Notes Series, 132 (1988).

[26] D. B. RAY, Reidemeister torsion and the Laplacian on lens spaces, Adv. Math. 4 (1970), 109-126.

[27] D. B. RAY And I. M. SingeR, R-torsion and the Laplacian on Riemannian manifolds, Adv. in Math. 7 (1971), 145-210.

[28] K. Reidemeister, Homotopieringe und Linseräume, Hamburger Abhandl. 11 (1935), 102-109. 
[29] G. Vincent, Les groupes linéaires finis sans points fixes, Commentarii Mathematici Helvetici, 20 (1947), 117-171.

[30] L. Weng And Y. You, Analytic torsions of spheres, Int. J. Math. 7 (1996), 109-125.

[31] J. A. WoLf, Spaces of constant curvature, McGraw-Hill Inc (1967).

[32] H. Zassenhaus, Über endliche Fastkörper, Abhandlungen aus dem Mathematischen Seminar der Hamburgischen Universität, 11 (1935), 187-220.

[33] H. Zassenhaus, The Theory of Groups, second edition, Chelsea Publishing Company, New York (1958). 

C

'curved join', 34

complexo de de Rham, 70

cone sobre $S^{n}, 56$

D

derivada

exterior $d, 69$

exterior adjunto $d^{\dagger}, 74$

determinante, 8

de Dieudonné, 5, 7, 10

não comutativo, $5,7,54$

E

elemento volume, 72

invariante, 72

espaço lenticular, 51, 61-66

F

forma

coexata, 74

cofechada, 74

de volume, 72

diferencial, 67

exata, 70

fechada, 70

harmônica, 75

forma espacial esférica, xi, 22

quaterniônica, 31, 46 função de Euler, 23

G

$(g, h)$-equivariante, 65

grupo

cíclico, 51

das transformações de $\mathfrak{F}_{\mathbb{C}}(G), 28$

de Whitehead, 4

de $G, 6$

de $R G, 6$

especial, 4

reduzido, 5

linear especial, 10

linear geral infinito, 3

livre de ponto fixo, 21

ortogonal, 20

quatérnios generalizado, xi, 19, 23, 31

quaterniônico, veja quatérnios generalizado

unitário, 20

$\mathbf{H}$

Hodge, $\star, 72$

\section{I}

'intertwining', 20

J

‘join’ $*, 61$ 
L

'lens', veja espaço lenticular

M

métrica, 71

Riemanniana, 50, 70

módulo

estavelmente livre, 7

trivial, 11

matriz elementar, 3

$\mathbf{P}$

pq-condição, 23

plano-pq, 34

produto

'wedge' $\wedge, 68$

exterior, 68

interno de formas, 73

tensorial, 67

\section{Q}

quatérnios

álgebra dos, 7
norma, 7
puros, 7
unitários, 8

quatérnios generalizado, xi, 19, 23, 31

quaterniônico, veja quatérnios generalizado

\section{$\mathbf{R}$}

$r$-forma, 67

R torção, xii, 11

absoluta, 12

da forma esp. esf. quaterniônica, 52, 55

do espaço lenticular, 51

relativa, 12 representação, 19

'faithful', 19

caractere da, 19

complexa, 20

conjugada, 21

de $G$ em $V, 19$

de dimensão finita, 19

dual, 19

equivalente, 19

a uma real, 20

ortogonalmente, 20

unitariamente, 20

espaço, 19

grau da, 19

irredutível, 19

absolutamente, 20

totalmente, 19

livre de ponto fixo, 21

ortogonal, 20

produto tensorial, 19

real, 20

sobre $\mathbb{F}, 19$

soma direta, 19

sub-representação, 19

própria, 19

unitária, 20

Riemanniana

métrica, 70

variedade, 70

RS torção, xii, 15

da esfera, 49

da forma esp. esf. quaterniônica, 55

do cone, 56,57

do disco, 58

do espaço lenticular, 51 


\section{S}

$S^{n}, 49,56$

^ de Hodge, 13, 72

soma de Einstein, 67

$\mathbf{T}$

tensor(es), 67, 70

teorema

de Cheeger-Müller, xii, 49

decomposição de Hodge, 75

torção

analítica, xii, 16

da esfera, 49

do disco, 58, 60

de Reidemeister, veja $\mathrm{R}$ torção

de Whitehead, veja $\mathrm{W}$ torção

$\mathrm{RS}$, veja RS torção

\section{V}

variedade

Riemanniana, 70

completa, xi, 22

W

W torção, xi, 11, 12 